\title{
Restrictions imposed by Superconformal Invariance On Quantum Field Theories
}

\author{
Shiraz Minwalla \\ Department of Physics \\ Princeton University \\ Princeton NJ 08544 \\ minwalla@princeton.edu
}

\begin{abstract}
We derive unitarity restrictions on the scaling dimensions of primary quantum operators in a superconformal quantum field theory, in $\mathrm{d}=3,4$, 5,6 .
\end{abstract}

\section{Introduction}

Whereas classical massless field theories are often conformally invariant, the same is rarely true of their quantum counterparts. To define a quantum field theory, you need to specify both a Lagrangian and a cutoff. The cutoff introduces a hidden dimensional scale in the definition of the theory. A scale transformation relates a quantum theory with a given cutoff to another quantum theory with the scaled cutoff. Therefore a QFT with nontrivial cutoff dependence (i.e., nonzero beta function) is never scale invariant, and so never conformally invariant. 
Since quantum field theories generically have nonzero beta function, a study of the implications of exact conformal invariance on a quantum field theory might seem to be of limited interest. That is, however, not the case. A QFT is indeed not scale invariant at arbitrary cut off, but as you lower the cut off in your theory, a truly gapless theory flows, in the Wilsonian sense, to a fixed point of the renormalization group - i.e., a point with zero beta function. Long wavelength physics in such a theory is thus governed by an effective quantum theory that is exactly scale invariant. In many examples this effective quantum field theory is a free massless theory. In some cases of interest however, the low energy effective theory is an interacting scale invariant, and generically conformally invariant [14] quantum field theory. Low energy effective theories completely specify the vacuum structure of a theory, and the nature of its massless excitations, and hence are of interest.

Supersymmetric field theories have proved to be particularly amenable to exact analysis in the infrared. Interacting gapless theories with unbroken supersymmetry typically exhibit both conformal and supersymmetric invariance in the infrared. The full symmetry of the (low energy effective) theory must then be generated by a superalgebra that contains in it, as sub super algebras, both the conformal algebra and the supersymmetry algebra. Such algebras are very constrained - in fact none exist in $d \geq 6$, and their possible forms are known in $d \leq 6$. These algebras are called superconformal algebras, and the symmetry they generate is called superconformal symmetry.

Three examples of interacting superconformal theories of current interest are the intrinsic theories living on the world volumes of coincident $M_{2}, M_{5}, D_{3}$ branes. A fourth example is the theory that governs the low energy behaviour of $N=1, d=4$ super QCD with $N_{f}$ quarks and $N_{c}$ colours with $\frac{3}{2} N_{c}<N_{f}<3 N_{c}$.

In this paper we will derive unitarity constraints on quantum field theories in $d=3,4,5,6$ that exactly implement the superconformal algebra. The constraints we will derive will take the form of inequalities that scaling dimensions of primary local operators in such a theory will be forced to obey.

This paper is arranged as follows.

In Section 2 we will determine constraints that conformal invariance imposes on a quantum theory in arbitrary dimension.

In section 3 we will construct the superconformal algebras that exist, i.e. list all commutation relations explicitly. We will also determine some constraints on scaling dimension, imposed by demanding the unitarity of 
these representations.

In section 4 we will review well known results in the theory of Lie Super Algebras, following Kac [7]. In particular we will review the classification of such algebras, and define explicitly all algebras that we will use in the rest of the paper. We will explicitly, following [8], identify the superalgebras corresponding to the superconformal algebras in $d \leq 6$, and verify that there are no superconformal algebras in $d>6$. We will proceed to use a method developed by Dobrev and Petkova in [3], [5], ,4] , (using the theory set up in [6] ), to almost completely determine the constraints imposed by unitarity on representations of these algebras. These constraints will determine the required inequalities on the scaling dimensions of local operators in a unitary QFT.

In section 5 we examine how our results fit into what is known about systems with superconformal invariance. We examine some primary operators in the world volume theory of the $M_{2}, D_{3}, M_{5}$ brane, and identify the representations that these operators label from the list of allowed representations generated in previous section. We then go on to review the oscillator construction [19] of the $d=6, n=2$ algebra, and explicitly construct the short representations of this algebra whose existence could not be conclusively established in section 4 .

In section 6 we present a detailed listing of all our results. The reader interested only in our results may jump directly to section 6 .

\section{The Conformal Group.}

In this section we will discuss the unitary representations of the conformal group in arbitrary dimension, as relevant to Quantum Field Theory. Our chief result will be the following. Primary fields in a conformally invariant QFT appear in multiplets that form representations of $S O(d)$. They also have a scaling dimension, $\epsilon_{0}$. Unitarity forces $\epsilon_{0}$ to obey an inequality of the form

$$
\epsilon_{0} \geq f(\text { representation of field) }
$$

where $f$ is a function we determine below. We will also obtain restrictions on the $S O(d)$ content of free operators in a conformal QFT. 


\subsection{Definition of the Conformal Group}

The conformal group in $d$ dimensions is generated by $\frac{d(d-1)}{2}$ Lorentz generators $M_{\mu \nu}, d$ momenta $P_{\mu}, d$ special conformal generators $K_{\mu}$ and a dilatation $D$. (Through this section greek indices run from 0 to $d-1$ ). These quantities obey commutation relations

$$
\begin{gathered}
{\left[M_{\mu \nu}, M_{\alpha \beta}\right]=(-i)\left[\eta_{\mu \beta} M_{\nu \alpha}+\eta_{\nu \alpha} M_{\mu \beta}-\eta_{\mu \alpha} M_{\nu \beta}-\eta_{\nu \beta} M_{\mu \alpha}\right]} \\
{\left[M_{\mu \nu}, P_{\alpha}\right]=(-i)\left[\eta_{\nu \alpha} P_{\mu}-\eta_{\mu \alpha} P_{\nu}\right]} \\
{\left[D, M_{\mu \nu}\right]=0} \\
{\left[M_{\mu \nu}, K_{\alpha}\right]=(-i)\left[\eta_{\nu \alpha} K_{\mu}-\eta_{\mu \alpha} K_{\nu}\right]} \\
{\left[D, P_{\mu}\right]=-i k_{\mu}} \\
{\left[D, K_{\mu}\right]=-i\left(-K_{\mu}\right)} \\
{[D, D]=0} \\
{\left[P_{\mu}, P_{\nu}\right]=0} \\
{\left[P_{\mu}, K_{\nu}\right]=(-i)\left[2 \eta_{\mu \nu} D+2 M_{\mu \nu}\right]} \\
{\left[K_{\mu}, K_{\nu}\right]=0}
\end{gathered}
$$

We are interested in representations of the conformal algebra in which all the generators above are implemented as hermitian operators. ${ }^{1}$

A representation of these commutation relations may be obtained through differential operators

$$
\begin{gathered}
M_{\mu \nu}=(-i)\left[x_{\mu} \partial_{\nu}-x_{\nu} \partial_{\mu}\right] \\
P_{\mu}=(-i)\left[\partial_{\mu}\right] \\
K_{\mu}=(-i)\left[-2 x_{\mu}(x . \partial)+x^{2} \partial_{\mu}\right] \\
D=(-i)[-x . \partial]
\end{gathered}
$$

The Conformal Group group is locally isomorphic to the group $S O(d, 2)$. Denote $S O(d, 2)$ generators by $S_{a b}$ where latin indices run from -1 to $d$. The

\footnotetext{
${ }^{1}$ It is easy to check that this is compatible with the commutation relations $2.3-2.11$, by checking that the later are invariant under conjugation.
} 
indices -1 and 0 are associated with -1 in the metric. The isomorphism of $S O(d, 2)$ and the standard conformal algebra is given by

$$
\begin{gathered}
S_{\mu \nu}=M_{\mu \nu} \\
S_{-1 d}=D \\
S_{\mu-1}=\frac{1}{2}\left[P_{\mu}+K_{\mu}\right] \\
S_{\mu d}=\frac{1}{2}\left[P_{\mu}-K_{\mu}\right]
\end{gathered}
$$

One may check that $S_{a b}$ thus defined obey commutation relations analogous to 2.2 , with $M s$ replaced by $S s$.

It is useful to define auxiliary quantities that obey the commutation rules of a Euclidian conformal algebra. Below indices $p$ and $q$ range from 1 to $d$.

$$
\begin{gathered}
M_{p q}^{\prime}=S_{p q} \\
D^{\prime}=(i) S_{-10} \\
P_{p}^{\prime}=\left[S_{p-1}+i S_{p 0}\right] \\
K_{p}^{\prime}=\left[S_{p-1}-i S_{p 0}\right]
\end{gathered}
$$

The commutation relations of these new objects are those of the generators of the Euclidian conformal group $S O(d+1,1)$. Primed objects have hermiticity properties derived from those of the normal generators

$$
\begin{aligned}
& M^{\prime \dagger}=M^{\prime} \\
& D^{\prime \dagger}=-D^{\prime} \\
& P^{\prime \dagger}=K^{\prime} \\
& K^{\prime \dagger}=P^{\prime}
\end{aligned}
$$

Of course the transition from the Lorentzian conformal group to the Euclidian conformal group was made with the help of $i \mathrm{~s}$, this shows up in the conjugation relations $2.24-2.27$. 


\subsection{Interpretation of Primed operators}

We will now give an interpretation of the primed operators we have defined in $2.20-2.23$, and explain why they, rather than the true generators of the algebra, will enter most of the calculations in this paper. Consider a Conformal QFT in $(d-1,1)$ dimensions. Consider a Euclidian continuation (Wick rotation ) of this theory. Quantize this Wick rotated theory radially, i.e., choose as surfaces of constant time the $d-1$ dimensional spheres centered around the origin. Interpret the sphere at infinity and at the origin respectively as the infinite future and the infinite past. The Euclidian QFT thus constructed has invariance under the conformal group $S O(d+1,1)$, whose generators we will call $P_{p}^{\prime}, M_{p q}^{\prime}, K_{p}^{\prime}{ }^{2}$ Here, for instance, $P_{m}^{\prime}$ is the operator that generates motion in the $m^{\text {th }}$ spatial direction (one of the $d$ Cartesian directions in our $d$ dimensional space).

Not all $S O(d+1,1)$ generators are Hermitian in this theory. Because the surfaces of 'constant time' are spheres, $M^{\prime}$ operators are guaranteed to be Hermitian and so obey 2.24. Since surfaces of constant ' $x$ ' are not equal time surfaces, $P^{\prime}$ operators are not necessarily Hermitian; in fact one may show that, (as in $d=2 \mathrm{CFTs}$ ), radial quantization imposes the Hermiticity condition $2.26,2.27$, on $P^{\prime}$ and $K^{\prime}$ operators (c.f. $L_{1}$ and $L_{-1}$ in $\mathrm{d}=2$ CFT). Similarly the 'Hamiltonian' of our theory, i.e., the generator of scale transformations $D^{\prime}$, is antihermitian (reflecting the fact that we are working in a Euclidianized theory), and so obeys 2.25 . In net, then, the Euclidian theory radially quantized has a symmetry group generated by $S O(d+1,1)$ generators that obey Hermiticity conditions $2.24-2.27$.

This is reassuring. The Lorentzian theory is invariant under $S O(d, 2)$, whereas its Wick rotation has invariance under $S O(d+1,1)$. This may worry the reader who is aware of the big difference between the unitary representations of $\mathrm{SO}(\mathrm{d}, 2)$ and $S O(d+1,1)$; merely from group theory it seems that the two theories describe the same physics. This 'paradox' has the obvious resolution; the Euclidian theory does not represent $S O(d+1,1)$ unitarily; generators of the radially quantized theory obey $2.24-2.27$, and $S O(d+1,1)$ with generators obeying these commutation relations is identical to $S O(d, 2)$ with unitary generators, definitions $2.20-2.23$ and consequent equations $2.24-2.27$ show.

Therefore, to study constraints imposed on the scaling dimensions of operators in a conformal QFT one may adopt two equally valid procedures. The first and most straightforward would be to study the Lorentzian theory -

\footnotetext{
${ }^{2}$ To forestall confusion we state explicitly that $P^{\prime}$ etc., are not related to the generators of symmetry of the Lorentz version of the same theory through $2.20-2.23$
} 
with an $S O(d, 2)$ symmetry unitarily implemented, and study the restrictions on the eigenvalues of operators under scalings $\mathrm{D}$, i.e., on $\epsilon_{0}$ defined by $[\mathrm{D}$, $\mathrm{Op}]=(-\mathrm{i}) \epsilon_{0}$ Op. A second, and equally valid procedure - the one we adopt in this paper - is to study the Euclidianized version of the theory that has $S O(d+1,1)$ symmetry implemented non-unitarily as in $2.24-2.27$, and study the restrictions on the eigenvalues of $D^{\prime}$ - interpreted as a scaling dimension of the operator in question only after a Wick rotation. That is we study restrictions on scaling dimensions $\epsilon_{0}$ of operators defined by $\left[D^{\prime}, O p^{\prime}\right]=(-i)\left(-i \epsilon_{0} O p^{\prime}\right)$.

\subsection{The structure of unitary representations}

Physically interesting irreducible unitary representations of the Conformal algebra are modules with energy bounded from below. ${ }^{3}$ The structure of such modules is described great detail in [1] for the special case $d=3$, and in [2] for the case $d=4$. Using the arguments in these papers one may deduce the following structural features of physically interesting modules in arbitrary dimension.

A unitary irreducible representation of the conformal group may be decomposed into a direct sum of irreducible representations of its maximal compact subgroup - $S O(d) \times S O(2)$. The $S O(d)$ here is generated by $M^{\prime}$, and the $S O(2)$ by $D^{\prime}$. No given $S O(d) \times S O(2)$ representation occurs more than finitely many times in this decomposition. The scaling dimension $\left(D^{\prime}\right.$ eigenvalue) of states in representations of physical interest are all of the form $\epsilon_{0}+\mathrm{n}$, where $\mathrm{n}$ is a positive integer. $\epsilon_{0}$ is the lowest dimension in the module, and is referred to as the scaling dimension of the module. The conformal module, if it is irreducible, contains exactly one $S O(d) \times S O(2)$ irreducible representation with dimension $\epsilon_{0}$. Let this representation have $S O(d)$ lowest weights $\left(-h_{1} . .-h_{[d / 2]}\right)$. These are referred to as the $S O(d)$ lowest weights of our module and the lowest $S O(d)$ weight vector in the $S O(d)$ irreducible representation with dimension $\epsilon_{0}$ is called the lowest weight vector of the conformal module. The full conformal module is uniquely determined by specifying its scaling dimension and its $S O(d)$ lowest weights. All states in the conformal module may be generated by acting on states of scaling dimension $\epsilon_{0}$ by $P^{\prime}$ operators (which raise the scaling dimension of states by unity).

\footnotetext{
${ }^{3}$ As we are interested in the implementation of this symmetry in a quantum theory, we actually describe irreducible representations of generators of the covering group. We will use notation loosely through the paper. In particular, when we speak of $S O(d)$ we always actually refer to its covering group
} 


\subsection{Implications for operators in a Conformal QFT}

In the previous subsection we described the structure of a unitary representation of the conformal algebra. It is composed of a module of states, and is completely characterized by a lowest weight state. We are interested in the application of this theory to the representations of the conformal algebra on the space of local quantum operators in a conformal QFT. The 'states' in our module will be local quantum operators. The action of conformal generators on these 'states' will be given by the commutator. Lowest weight operators will occur in multiplets that fall in an irreducible representation of $S O(d) \times S O(2)$. Lowest weight operators (or primary operators, as we will sometimes call them) commute with $K^{\prime}$ (lowering) operators. All operators in a given module may be obtained by commuting primary operators with $P^{\prime} s$.

We can be more explicit. Consider a multiplet of local operators, $\phi_{\alpha}(x)$ in a conformally invariant QFT. The $\mathrm{x}$ dependence of these operators can be solved for; $\phi_{\alpha}(x)=e^{-i P . x} \phi_{\alpha}(0) e^{i P . x}$. (Here $P$ is the generator of translations in our space. In the Euclidian theory $P$ should be replaced by $\left.P^{\prime}\right)$. Differentiating this expression one obtains $\left[P_{\mu}, \phi_{\alpha}(x)\right]=(-i)\left(-\partial_{\mu} \phi_{\alpha}(x)\right)$. We may also determine the form of the commutator of $\phi_{\alpha}(x)$ with the other generators of the conformal group. ${ }^{4}$ We specify the transformation properties of our multiplet by setting $\left[M_{\mu \nu}, \phi_{\alpha}(0)\right]=\left(-M_{\mu \nu}^{R}\right)_{\alpha}^{\beta} \phi_{\beta}(0)$, and $\left[D, \phi_{\alpha}(0)\right]=\epsilon_{0} \phi_{\alpha}(0)$, where $M^{R}$ is a the Lorentz Transformation operator in the $\mathrm{R}$ representation - the representation $\phi$ happens to transform in and $\epsilon_{0}$ is the scaling dimension of $\phi$. The action of conformal generators on operator $\phi$ is then given by differential/ matrix operators acting from the left as

$$
\begin{gathered}
M_{\mu \nu}=(i)\left[x_{\mu} \partial_{\nu}-x_{\nu} \partial_{\mu}\right]-M_{\mu \nu}^{R} \\
P_{\mu}=(i)\left[\partial_{\mu}\right] \\
K_{\mu}=(i)\left[-2 x_{\mu}(x . \partial)+x^{2} \partial_{\mu}\right]-2 x^{\alpha} M_{\alpha \mu}^{R}+2 x_{\mu} \epsilon_{0} \\
D=(i)[-x . \partial]+\epsilon_{0}
\end{gathered}
$$

The difference in signs between $2.28-2.30$ and $2.12-2.14$ is the usual coset space artifact (derivatives act from the 'inside' rather than the 'outside'). A primary operator is killed by the $K\left(K^{\prime}\right.$ in the Euclidian theory) differential operator above.

\footnotetext{
${ }^{4}$ Let $T_{a}$ stand for the set of generators of the conformal algebra. Let $\left[T_{a}, e^{-i P \cdot x}\right]=$ $t_{a} e^{-i P . x}+e^{-i P . x} \beta_{a}^{b} T_{b}$ (where $t_{a}$ is a hermitian differential operator and $\beta$ a matrix). Then $\left[T_{a}, \phi_{\alpha}(x)\right]=t_{a} \phi_{\alpha}(x)+e^{-i P \cdot x}\left[T_{a}+\beta_{a}^{b} T_{b}, \phi_{\alpha}(0)\right] e^{i P . x}$.
} 


\subsection{Restrictions imposed by Unitarity}

In this subsection we will derive restrictions placed by unitarity on the scaling dimension of an acceptable module as a function of its highest $S O(D)$ weights.

Let $|\{s\}\rangle \quad\left(\{s\}=\left\{s_{1}, s_{2}, \ldots s_{n}\right\}\right.$ where $n=[d / 2], s$ 's are $S O(d)$ weights $)$, represent the kets of the $S O(d)$ irreducible representation that contains the lowest weight state of our module. All the states above have $-\mathrm{i} D^{\prime}=\epsilon_{0}$. Let the representation hosted by $|\{s\}\rangle$ have highest weights $\mathrm{h}=\left\{h_{1}, h_{2}, \ldots h_{n}\right\}$.

Consider the states $P_{\mu}^{\prime} \mid\{s\}>$. This is the set of states in the with dimension $\epsilon_{0}+1$. If our module is unitary, arbitrary linear combinations of these states must have positive norm. Using the fact that $P^{\prime \dagger}=K^{\prime}$, the above condition is equivalent to demanding that the matrix

$$
A_{\mu\{s\}, \nu\{t\}}=<\{s\}\left|K_{\mu}^{\prime} P_{\nu}^{\prime}\right|\{t\}>
$$

is positive, i.e., has only non negative eigenvalues. Using the group commutation relations 2.10 , and $D^{\prime}=(-i) \epsilon_{0}$

$$
A_{\mu\{s\}, \nu\{t\}}=2<\{s\}\left|\epsilon_{0}+(-i) M_{\mu \nu}^{\prime}\right|\{t\}>
$$

Positivity of $\mathrm{A}$ is then the condition that the matrix

$$
B_{\mu\{s\}, \nu\{t\}}=<\{s\}\left|(-i) M_{\mu \nu}^{\prime}\right|\{t\}>
$$

has no eigenvalue smaller than $-\epsilon_{0}$.

To process this condition we use a trick. Notice

$$
(-i) M_{\mu \nu}^{\prime}=\frac{1}{2}(-i)\left(\delta_{\mu \alpha} \delta_{\nu \beta}-\delta_{\mu \beta} \delta_{\nu \alpha}\right) M_{\alpha \beta}^{\prime}
$$

That is ${ }^{5}$

$$
M_{\mu \nu}^{\prime}=\left(V \cdot M^{\prime}\right)_{\mu \nu}
$$

Where $\left(V_{\alpha \beta}\right)_{\mu \nu}=(-i)\left(\delta_{\mu \alpha} \delta_{\nu \beta}-\delta_{\mu \beta} \delta_{\nu \alpha}\right)$ are the $S 0(d)$ generators in the vector representation, and $A \cdot B$ is defined as $\frac{1}{2} A_{\alpha \beta} B_{\alpha \beta}$, for $\mathrm{A}$ and $\mathrm{B}$ generators of $S O(d)$ in arbitrary representations, both sides of this definition being operators (matrices) on the tensor product of the two representation spaces. Therefore

$$
B_{\mu\{s\}, \nu\{t\}}=\left(V \cdot M^{\prime}\right)_{\mu\{s\}, \nu\{t\}}
$$

\footnotetext{
${ }^{5}$ In the equation below $M$ is an operator, $V$ is a matrix, $\mu$ and $\nu$ are its indices.
} 
Now

$$
V \cdot M^{\prime}=\frac{1}{2}\left(\left(V+M^{\prime}\right) \cdot\left(V+M^{\prime}\right)-V \cdot V-M^{\prime} \cdot M^{\prime}\right)
$$

Determining the allowed eigenvalues of this operator is now analogous to the problem of determining the allowed values of $L \cdot S$ for the hydrogen atom. One may perform a similarity transformation on our matrix $B$ that corresponds to moving to a Clebsch Gordan coupled basis in the tensor product space. In such a basis each of $\left(V+M^{\prime}\right)^{2}, V^{2}$, and $M^{\prime 2}$ are 'good quantum numbers'.

Let the $M^{\prime}$ matrices transform in representation $R$, and let $R^{\prime}$ be the representation with smallest quadratic Casimir ${ }^{6}$ that appears in the Clebsch Gordan expansion of $R \times V$. Then the condition 2.34 is

$$
\epsilon_{0} \geq \frac{1}{2}\left(c_{2}(R)+c_{2}(V)-c_{2}\left(R^{\prime}\right)\right)
$$

To process this equation it is helpful to note the following formula for the quadratic casimir of $S O(d)$ in terms of its highest weights

$$
c_{2}(\{h\})=\sum_{i=1}^{[d / 2]}\left(h_{i}^{2}+(d-2 i) h_{i}\right)
$$

as well as the following special cases; $c_{2}$ (scalar $)=0, c_{2}$ (spinor $)=$ $(d / 8)(d-1), c_{2}$ (vector $)=d-1$. The highest weights of $R^{\prime}$ in terms of those of $R$ may be deduced from appendix 2 and the condition above made more explicit. For instance, if the representation $\mathrm{R}$ has highest wts s.t. $h_{1} \geq\left|h_{2}\right|+1$ then the condition above becomes $\epsilon_{0} \geq h_{1}+d-2$. The formula for an arbitrary representation in terms of its highest weights is

$$
\epsilon_{0} \geq\left|h_{i}\right|+d-i-1
$$

where $i$ is the smallest value s.t. $h_{i} \geq\left|h_{i+1}\right|+1$, provided there is such an $i$. If no such $i$ exists then all lowest weights have equal modulus value. In the case that they are all equal to zero the condition is merely $\epsilon_{0} \geq 0$. If they are all equal and $\geq 1$ then the condition above applies with $i=[d / 2]$. The only remaining case is that of spinor representations for which the answer is $\epsilon_{0} \geq(d-1) / 2$. The answer for the vector representation is $\epsilon_{0} \geq d-1$. We make some special cases explicit below

\footnotetext{
${ }^{6} c_{2}\left(R^{\prime}\right)=(\operatorname{defn}) M . M\left(\right.$ in the $R^{\prime}$ rep $)$
} 
In $d=3$ representations of $S O(d)$ are labeled by a half integer $j$. The condition above is simply

$$
\begin{gathered}
\epsilon_{0} \geq 0 \quad(j=0) \\
\epsilon_{0} \geq 1 \quad(j=1 / 2) \\
\epsilon_{0} \geq j+1 \quad(j \geq 1)
\end{gathered}
$$

These are exactly ${ }^{7}$ the conditions derived in [1] . In $d=4 S O(4)=$ $S U(2) \times S U(2)$, and so representations are labeled by two half integers, $j_{1}$ and $j_{2}$. The conditions derived for this case is

$$
\epsilon_{0} \geq f\left(j_{1}\right)+f\left(j_{2}\right)
$$

Where $f(j)$ is defined by

$$
f(j)=0 \text { for } j=0, \quad f(j)=j+1 \text { for } j>0
$$

These are precisely ${ }^{8}$ the conditions derived in [2] .

In $d=5$ representations are labeled by $S O(5)$ highest weights $h_{1}$ and $h_{2}$. The conditions are

$$
\begin{gathered}
\epsilon_{0} \geq 0 \quad\left(h_{1}=h_{2}=0\right) \\
\epsilon_{0} \geq 2 \quad\left(h_{1}=h_{2}=\frac{1}{2}\right) \\
\epsilon_{0} \geq h+2 \quad\left(h_{1}=h_{2}=h \neq 0, \frac{1}{2}\right) \\
\epsilon_{0} \geq h_{1}+3 \quad\left(h_{1}>h_{2}\right)
\end{gathered}
$$

In $d=6$ representations are labeled by $S O(6)$ highest weights $h_{1}, h_{2}$ and $h_{3}$. The conditions are

$$
\begin{gathered}
\epsilon_{0} \geq 0 \quad\left(h_{1}=h_{2}=h_{3}=0\right) \\
\epsilon_{0} \geq h+2 \quad\left(h_{1}=h_{2}=\left|h_{3}\right|=h \neq 0\right) \\
\epsilon_{0} \geq h+3 \quad\left(h_{1}=h_{2}>\left|h_{3}\right|\right) \\
\epsilon_{0} \geq h_{1}+4 \quad\left(h_{1}>h_{2}\right)
\end{gathered}
$$

In an arbitrary dimension $d$ special representations obey

$$
\epsilon_{0} \geq 0 \quad \text { (scalar) }
$$

\footnotetext{
${ }^{7}$ These results supplemented with the level 2 condition ahead for the $j=0$ case

${ }^{8}$ These results supplemented with the level 2 condition ahead for the $j=0$ case
} 


$$
\begin{gathered}
\left.\epsilon_{0} \geq(d-1) / 2 \quad \text { (spinor }\right) \\
\left.\epsilon_{0} \geq(d-1) \quad \text { (vector }\right)
\end{gathered}
$$

All these results make eminent sense. Since the identity operator in our field theory constitutes a singleton scalar representation with $\epsilon_{0}=0$ we could not have expected a stronger bound for the scalar case. ${ }^{9}$

The operator that saturates the bound for the spinor representation gives zero on contracting with $P_{\mu}^{\prime}$ in such a manner as to get the smallest resultant representation. Therefore this operator obeys $\left[\not^{\prime}, \psi\right]=0$, i.e., the Dirac equation. Therefore our bound is saturated by the Free Dirac field, and indeed the scaling dimension above is the canonical dimension for such a field.

The bound on the vector representation is, at first sight, a little puzzling. In 4 dimensions, for instance, it is $\epsilon_{0} \geq 3$, this seems to be in contradiction with the fact that the scaling dimension of the vector field in Maxwell theory is 1. The contradiction is explained away by noting that the Maxwell $A_{\mu}$ field is not gauge invariant, and so is not represented in a theory with only positive norm states. Our restrictions apply only to gauge invariant operators. Vector operators that saturate the bound above satisfy $\left[P_{\mu}, \psi_{\mu}\right]=0$; examples of such operators are conserved currents; these are indeed vectors, and indeed have the scaling dimension above.

We have obtained constraints from a unitarity analysis at level one. New constraints may well emerge at level ${ }^{10} 2$ and higher. The level 2 analysis is particularly easy to carry out for a lowest weight state in the scalar representation and yields

$$
\left.\epsilon_{0}\left(\epsilon_{0}-(d-2) / 2\right) \geq 0 \text { (scalar }\right) .
$$

This condition permits the singleton representation with $\epsilon_{0}=0$, but forces all non singleton scalar representations to have dimension greater than the scaling dimension of the free scalar field.

We now have a set of necessary conditions on scaling dimensions of primary operators in a unitary conformal quantum field theory. Nothing we

\footnotetext{
${ }^{9}$ Note that any operator that has $\epsilon_{0}=0$ is annihilated by (i.e., commutes with) the momentum operator $P_{\mu}^{\prime}$, and so is translationally invariant. The identity operator is the only translationally invariant local operator, and so it constitutes the unique representation with $\epsilon_{0}=0$

${ }^{10}$ The level of $|\psi\rangle$ is the number of $P^{\prime}$ operators that act on the lowest weight state to give $|\psi\rangle$. When we deal with the superconformal algebra, 'level' will have the same meaning but with $P^{\prime}$ replaced by $Q^{\prime}$.
} 
have said so far has given us any reason to suspect that this is also a sufficient set of conditions. However comparison with the extensive analysis carried out in [1] and [2] show that the conditions we have turn out to be both necessary and sufficient to ensure unitarity in $d=3,4$. This may or may not be a coincidence of low dimensions. It would be interesting - and seemingly not too difficult - to attempt to generalize Mack's construction of explicitly unitary representations in [2] to higher dimensions, and so obtain sufficient conditions for unitarity.

\subsection{Free Conformally invariant fields}

One often thinks of a conformally invariant theory as a theory of massless particles. Since we know that free massless particles in d dimensions appear in $S O(d-2)$ multiplets, it is perhaps a little puzzling that operators in a conformally invariant theory appear in multiplets of $S O(d)$.

To understand this start with $2.28-2.31$ for the action of conformal generators on an arbitrary $S O(d)$ multiplet of operators. Impose the condition $P . P \phi_{\alpha}(x)=0$ on all operators in the multiplet. Since this equations is true for all $\phi \mathrm{s}$ in the multiplet, making an infinitesimal conformal transformation on any specific $\phi$ gives $P . P T_{a} \phi_{\alpha}(x)=0$ for all conformal generators $T_{a}$. Which means that

$$
\left[P . P, T_{a}\right] \phi_{\alpha}(x)=0
$$

for all conformal generators $T_{a}$. Note that P.P is not a casimir of the conformal group.

Setting $T_{a}=P_{\mu}, M_{\mu \nu}, D$ in 2.59 yields trivialities. Setting $T_{a}=K_{\mu}$ and using $2.28-2.31$ yields the following restriction on $\phi_{\alpha}(p)$ (we have fourier transformed the operator).

$$
p_{\mu}\left(-i M_{\mu \nu}^{R}\right)_{\alpha}^{\beta} \phi_{\beta}=\left(\frac{d-2}{2}-\epsilon_{0}\right) p_{\nu} \phi_{\alpha}
$$

To analyze this equation get into a frame in which $p_{\mu}=(1,1,0,0,0 . .0)$, where the components in ( ) are time, the first spatial component, ... . 2.60 is $d$ equations because of the free index $\nu$. When $\nu=2,3$..d- 1 , the RHS of 2.60 is zero, and the equation becomes

$$
\left(M_{1 i}^{R}-M_{0 i}^{R}\right) \phi=0
$$

$M s$ here are matrices representing $S O(d-1,1)$. Convert these to $S O(d)$ 
generators by $M_{\mu \nu}^{\text {lorentz }}=(-i)^{n} M_{\mu \nu}^{\text {Euclid }}$, where $\mathrm{n}$ is the number of zeroes in $\mu, \nu$. Let the first weight of our $S O(d)$ algebra be that associated with $M_{01}^{R}$, 2.61 says that any $M^{R}$ that raises the first weight of $\phi$ and does absolutely anything to the other weights kills $\phi$. That is, $\phi$ is a state of highest first weight in the $S O(d)$ multiplet, but whose 2 nd to $[d / 2]^{\text {th }}$ weights can take any value in the multiplet. When we set $\nu=0$ or 12.60 becomes $(-i) M_{01}^{R} \phi=$ $\left(\frac{d-2}{2}-\epsilon_{0}\right) \phi$. Since $M_{0 i}^{\text {lorentz }}=(-i) M_{0 i}^{\text {Euclid }}=H_{1}\left(H_{1}\right.$ is the first Cartan generator of $S O(d))$, we obtain

$$
\epsilon_{0}=h_{1}+\frac{d-2}{2}
$$

where $h_{1}$ is the highest first weight of $\phi$. The analysis above has given us an equation for the scaling dimension of $\phi$ as a function of its $S O(d)$ representation. It has also made clear the connection between the $S O(d)$ representation of the conformal operator, and the $S O(d-2)$ little group representation of the particle it creates. Explicitly, for a free conformal operator in momentum space, the $S O(d)$ weight connected with rotations in the momentum - time plane is restricted to be the highest weight in the $S O(d)$ representation. The remaining weights of $\phi$ are arbitrary, and in fact fill out a multiplet of $S O(d-2)$ - the little group of the massless Lorentz algebra. Therefore a free conformally invariant field (if one such exists-see below) with $S O(d)$ highest weights $h_{1}, h_{2} . . h_{[d / 2]}$ corresponds to a particle transforming in $h_{2}, h_{3}, \ldots h_{[d / 2]}$ of its $S O(d-2)$ little group. For instance, an $S O(d)$ scalar is an $S O(d-2)$ scalar, an $S O(d)$ spinor of given chirality is an $S O(d-2)$ spinor of the same chirality, an $S O(d)$ vector is an $S O(d-2)$ scalar, and an $S O(d)$ antisymmetric tensor is an $S O(d-2)$ vector. (We will actually see that free representations corresponding to the last 2 do not exist.)

We have noted above that a multiplet $\phi_{\alpha}$ that obeys $P . P \phi_{\alpha}(x)=0$ has also, for consistency, to obey $\left[P . P, T_{a}\right] \phi_{\alpha}(x)=0$. That is not the only consistency condition, however. One may iterate the argument above to generate the conditions

$$
\left[\left[P . P, T_{a}\right], T_{b}\right] \phi_{\alpha}(x)=0
$$

for arbitrary conformal generators $T_{a}, T_{b}$, and so on. These equations yield new equations for $\epsilon_{0}$ which may or may not be consistent with 2.62 . An inconsistency would rule out the existence of a free operator in a given representation. Choosing $T_{a}, T_{b}$ above to be $K_{\alpha}, K_{\beta}$ and using $2.28-2.31$ we obtain ${ }^{11}$

$$
\left(-M_{\beta \mu}^{R} M_{\mu \alpha}^{R}-M_{\alpha \mu}^{R} M_{\mu \beta}^{R}\right) \phi=\eta_{\alpha \beta}\left(2 \epsilon_{0}\right)\left(\epsilon_{0}-\frac{d-2}{2}\right) \phi
$$

\footnotetext{
${ }^{11}$ This equation was obtained in [?] by different means
} 
2.65 may be processed in detail as an eigenvalue equation, but for our purposes it is sufficient to trace it over $\alpha$ and $b$ indices. This yields

$$
\epsilon_{0}\left(\epsilon_{0}-\frac{d-2}{2}\right)-\frac{2}{d} c_{2}(R)=0
$$

an equation that could also have been obtained by employing the techniques of the previous section by setting $<\{s\}\left|\left(K^{\prime} \cdot K\right)\left({ }^{\prime} P^{\prime} \cdot P^{\prime}\right)\right|\{t\}>=0$. We may now ask under what circumstances 2.62 and 2.65 are consistent. It is easy to check that this is the case only when $h_{1}=h_{2}=h_{3}=\ldots=h_{[d / 2]}=h$ for any $h$ (when d is even), and when $h_{1}=h_{2}=h_{3}=. .=\left|h_{[d / 2]}\right|=h$ for $h=0$ or $\frac{1}{2}$ (when d is odd). These constraints on possible free representations were obtained in [13].

\section{The Super Conformal Algebras}

The structure of the supersymmetry algebra in arbitrary dimension is well known [12]. While minor details of the algebra vary with dimension, the supersymmetry generators $Q_{\alpha}$ always transform as spinors of the Lorentz Group. In a conformally invariant theory the $(d-1,1)$ Lorentzian Spinor $Q_{\alpha}$ forms a part of (is completed to) a $(d, 2)$ conformal spinor.

Notice that $(d-1,1)$ and $(d, 2)$ spinors have identical reality properties. In fact a $(d, 2)$ conformal spinor with specific reality properties is composed of two $(d-1,1)$ Lorentzian spinors of the same reality properties. This can be made explicit by the following choice of $\Gamma$ matrices for $S O(d, 2){ }^{12}$

$$
\begin{gathered}
\Gamma_{\mu}=\left(\begin{array}{cc}
\sigma_{\mu} & 0 . \\
0 & -\sigma_{\mu} .
\end{array}\right) \\
\Gamma_{-1}=\left(\begin{array}{cc}
0 & -I . \\
I & 0 .
\end{array}\right) \\
\Gamma_{d}=\left(\begin{array}{cc}
0 & I . \\
I & 0 .
\end{array}\right) \\
\Gamma_{d+1}=\left(\begin{array}{cc}
\sigma_{d+1} & 0 . \\
0 & -\sigma_{d+1} .
\end{array}\right)
\end{gathered}
$$

${ }^{12} S O(d-1,1) \Gamma$ matrices are denoted by $\sigma$ through the rest of this paper. 
The last matrix exists only for even $d . Q$ is completed to give a full conformal spinor $\mathrm{V}$ with the introduction of a new Lorentz spinor $S$ as

$$
V=\left(\begin{array}{c}
Q_{\alpha} . \\
C_{\theta \phi} \bar{S}^{\phi} .
\end{array}\right)
$$

$\mathrm{C}$ is the charge conjugation matrix. We set ${ }^{13}$

$$
\left[S_{a b}, V_{\alpha}\right]=R\left(M_{a b}\right)_{\alpha}^{\beta} V_{\beta}
$$

With $R\left(M_{a b}\right)=(i / 4)\left[\Gamma_{a}, \Gamma_{b}\right]$. Specifically

$$
\begin{gathered}
R\left(P_{\mu}\right)=(-i)\left(\begin{array}{cc}
0 & 0 \\
\sigma_{\mu} & 0
\end{array}\right) \\
R\left(K_{\mu}\right)=(-i)\left(\begin{array}{cc}
0 & \sigma_{\mu} \\
0 & 0
\end{array}\right) \\
R(D)=(-i / 2)\left(\begin{array}{cc}
I & 0 \\
0 & -I
\end{array}\right) \\
R\left(M_{\mu \nu}\right)=\left(\begin{array}{cc}
R\left(m_{\mu \nu}\right) & 0 \\
0 & R\left(m_{\mu \nu}\right)
\end{array}\right)
\end{gathered}
$$

Where $R\left(m_{\mu \nu}\right)=(+i / 4)\left[\sigma_{\mu}, \sigma_{\nu}\right]^{14}$

When working with the conformal group we had found it useful to define auxiliary (primed, non hermitian) generators $(2.20-2.23)$ that obeyed the commutation relations of generators of the Euclidian conformal group. We interpreted these operators physically in subsection 2.2. We will now define objects that will be similarly useful, and that have an equivalent interpretation in the full superconformal group; auxiliary odd generators $Q^{\prime}$ and $S^{\prime}$, objects that transform as Euclidian spinors under our auxiliary (primed) conformal generators. Define ${ }^{15}$

$$
\begin{aligned}
& Q^{\prime}=\frac{1}{\sqrt{2}}\left(Q-i \sigma_{0} S\right) \\
& S^{\prime}=\frac{1}{\sqrt{2}}\left(Q+i \sigma_{0} S\right)
\end{aligned}
$$

\footnotetext{
${ }^{13}$ Recall that if $\left[M^{i}, Q_{\alpha}\right]=G_{\alpha \beta}^{i} Q_{\beta}$ then the commutation relations between the operators $M^{i}$ are the same as those between the matrices $G^{i T}$ (not $G^{i}$ ), i.e., those between $-G^{2}$. We have thus chosen $\mathrm{G}$ to be -(matrices that have standard $\mathrm{M}$ commutation relations).

${ }^{14}$ Notice this means that $Q$ and $C \bar{S}$ each transform as spinors under the Lorentz group, have dilatation wt $\frac{1}{2}$ and $-\frac{1}{2}$ respectively, and, schematically, $[P, Q]=[K, C \bar{S}]=0$, $[K, Q]=C \bar{S},[P, C \bar{S}]=Q$.

${ }^{15}$ The fact $Q$ is a chiral spinor makes the definition of $Q^{\prime}$ and $S^{\prime}$ different in $\mathrm{d}=6$. In that dimension as in all others $Q^{\prime}$ and $S^{\prime}$ are defined so as to make $M^{\prime}$ and $D^{\prime}$ block diagonal in the $Q^{\prime}, S^{\prime}$ basis.
} 
The action of $M^{\prime}, P^{\prime}, K^{\prime}$ etc., on $Q^{\prime}$ and $S^{\prime}$ may now be determined.

$$
\begin{gathered}
{\left[M_{p q}^{\prime}, Q_{\alpha}^{\prime}\right]=(i / 4)\left[\Gamma_{p}, \Gamma_{q}\right]_{\alpha}^{\beta} Q_{\beta}^{\prime}} \\
{\left[M_{p q}^{\prime}, S_{\widetilde{\alpha}}^{\prime}\right]=(i / 4)\left[\widetilde{\Gamma}_{p}, \widetilde{\Gamma}_{q}\right]_{\widetilde{\alpha}}^{\widetilde{\beta}} Q_{\widetilde{\beta}}^{\prime}} \\
{\left[D^{\prime}, Q_{\alpha}^{\prime}\right]=(-i / 2) Q_{\alpha}^{\prime}} \\
{\left[D^{\prime}, S_{\widetilde{\alpha}}^{\prime}\right]=(-i / 2)-S_{\widetilde{\alpha}}^{\prime}} \\
{\left[P_{p}^{\prime}, Q_{\alpha}^{\prime}\right]=0} \\
{\left[K_{p}^{\prime}, S_{\widetilde{\alpha}}^{\prime}\right]=0} \\
{\left[P_{p}^{\prime}, S_{\widetilde{\alpha}}^{\prime}\right]=-\left(\widetilde{\Gamma}_{p} \sigma_{0}\right)_{\widetilde{\alpha}}^{\beta} Q_{\beta}} \\
{\left[K_{p}^{\prime}, Q_{\alpha}^{\prime}\right]=+\left(\Gamma_{p} \sigma_{0}\right)_{\alpha}^{\widetilde{\beta}} S_{\widetilde{\beta}}}
\end{gathered}
$$

Where ${ }^{16}$

$$
\begin{array}{cc}
\Gamma_{i}=\sigma_{i} & \Gamma_{d}=-i \sigma_{0} \\
\widetilde{\Gamma_{i}}=\sigma_{i} & \widetilde{\Gamma_{d}}=+i \sigma_{0}
\end{array}
$$

To complete the superconformal algebra, we now need to specify anticommutation relations between various $Q s$ and $S s$. In general we deal with extended susy and so should also add R symmetry indices to $Q s$ and $S s$, and specify their commutation relations with generators of $\mathrm{R}$ symmetry also.

Using well known $Q-Q$ and $R-Q$ (anti)commutation relations [12], it is straightforward to deduce the commutation relations of $S$ with conformal, $\mathrm{R}$ symmetry and $Q$ generators, using Jacobi identities. Rather surprisingly, it turns out not to be possible to satisfy these Jacobi identities in arbitrary dimensions. Satisfaction of the identities requires certain $\Gamma$ matrix identities, which are true only in low dimensions. Superconformal algebras exist only in $d \leq 6$.

We will explain this fact from the theory of Lie Super Algebras presented in the next subsection. In the rest of this subsection, we complete our listing of the commutation relations for the superalgebras that do exist.

$$
\mathrm{d}=\mathbf{3}
$$

\footnotetext{
${ }^{16} \Gamma$ and $\widetilde{\Gamma}$ matrices each generate a Euclidian Clifford algebra in $d$ dimensions. $Q^{\prime}$ transforms as a spinor in the $\Gamma$ representation, and $S^{\prime}$ as a spinor in the $\widetilde{\Gamma}$ representation. Notice that in a dimension in which Lorentzian $\sigma$ matrices may all be chosen real, (and making such a choice), the two sets of $\Gamma$ matrices above are complex conjugates of each other, and so $Q^{\prime}$ and $S^{\prime}$ transform in complex conjugate representations. Notice also that $\sigma_{0}$ interpolates between ordinary and tilde indices, as is clear from its commutation properties with $\Gamma$ and $\widetilde{\Gamma}$.
} 
$S O(2,1)$ admits a set of real $\sigma$ matrices. We work in this basis. As in [12], our $\sigma$ matrices are also chosen to be hermitian, except $\sigma_{0}$ which is antihermitian. The charge conjugation matrix is defined by the equation $C^{-} 1 \sigma C=-\sigma^{T}$. With our choice of $\sigma_{\mu}, \sigma_{0}$ solves this equation, and so may be identified with C. The spinors $Q$ and $S$ are both real; in our special basis this means

$$
\begin{gathered}
Q^{\dagger}=Q \quad ; S^{\dagger}=S \\
Q^{\prime \dagger}=S^{\prime} \quad ; S^{\prime \dagger}=Q^{\prime}
\end{gathered}
$$

The algebra exists with an arbitrary number $\mathrm{n}$ of susy generators, which are distinguished by an $\mathrm{R}$ symmetry label $i$. We sometimes suppress indices of $Q_{i \alpha}$ depending on context. The R symmetry group is $S O(n)$; its generators are antisymmetric tensors $I_{i j}$ that obey

$$
\begin{gathered}
{\left[I_{i j}, I_{m n}\right]=(-i)\left[I_{i n} \delta_{j m}+I_{j m} \delta_{i j}-I_{i m} \delta_{j n}-I_{j n} \delta_{i m}\right]} \\
{\left[I_{i j}, Q_{m}\right]=(-i)\left[Q_{i} \delta_{j m}+-Q_{j} \delta_{i m}\right]} \\
{\left[I_{i j}, Q_{m}^{\prime}\right]=(-i)\left[Q_{i}^{\prime} \delta_{j m}+-Q_{j}^{\prime} \delta_{i m}\right]} \\
{\left[I_{i j}, S_{m}\right]=(-i)\left[S_{i} \delta_{j m}+-S_{j} \delta_{i m}\right]} \\
{\left[I_{i j}, S_{m}^{\prime}\right]=(-i)\left[S_{i}^{\prime} \delta_{j m}+-S_{j}^{\prime} \delta_{i m}\right]} \\
{\left[I_{i j}, M_{p q}\right]=0}
\end{gathered}
$$

Odd elements anticommute according to

$$
\begin{gathered}
\left\{Q_{i \alpha}, Q_{j \beta}\right\}=(\not P C)_{\alpha \beta} \delta_{i j} \\
\left\{S_{i \alpha}, S_{j \beta}\right\}=(\not \not C)_{\alpha \beta} \delta_{i j} \\
\left\{Q_{i \alpha}, S_{j \beta}\right\}=\frac{\delta_{i j}}{2}\left[\left(M_{\mu \nu} \Gamma_{\mu} \Gamma_{\nu} C\right)_{\alpha \beta}+2 D C_{\alpha \beta}\right]-C_{\alpha \beta} I_{i j}
\end{gathered}
$$

Primed odd variables obey similar equations

$$
\begin{gathered}
\left\{Q_{i \alpha}^{\prime}, Q_{j \beta}^{\prime}\right\}=\left(\not^{\prime} C\right)_{\alpha \beta} \delta_{i j} \\
\left\{S_{i \widetilde{\alpha}}^{\prime}, S_{j \widetilde{\beta}}^{\prime}\right\}=\left(\widetilde{\not}{ }^{\prime} C\right)_{\widetilde{\alpha} \widetilde{\beta}} \delta_{i j} \\
\left\{Q_{i \alpha}^{\prime}, S_{j \widetilde{\beta}}^{\prime}\right\}=i \frac{\delta_{i j}}{2}\left[\left(M_{\mu \nu}^{\prime} \Gamma_{\mu} \Gamma_{\nu} C\right)_{\alpha \widetilde{\beta}}+2 D^{\prime} \delta_{\alpha \widetilde{\beta}}\right]-(i) \delta_{\alpha \widetilde{\beta}} I_{i j} \\
\mathbf{d}=4
\end{gathered}
$$

$\mathrm{d}=4$ is very similar to $\mathrm{d}=3$. Our $\sigma$ matrices are chosen to be real and hermitian (antihermitian for $\sigma_{0}$ ). Charge conjugation is defined as for $\mathrm{d}=3$, 
and once again $\sigma_{0}$ fits the bill. In our Majorana basis 4 component Majorana spinors $Q$ and $S$ are both real;

$$
\begin{array}{cc}
Q^{\dagger}=Q & ; S^{\dagger}=S \\
Q^{\prime \dagger}=S^{\prime} & ; S^{\prime \dagger}=Q^{\prime}
\end{array}
$$

The R symmetry in $\mathrm{d}=4$ is $U(n)$ ( $\mathrm{n}=$ the number of Qs) which acts differently on the positive and negative chirality parts of $Q$. The algebra exists for arbitrary $n$.

We define $\left.P_{ \pm}=\left(I \pm \sigma_{5}\right) / 2\right)$. Note that $\left(P_{+}\right)^{T}=P_{-} ; P_{+}^{*}=P_{-}$; $\left(P_{+}\right)^{\dagger}=P_{+}$. Commutation relations between $U(n)$ generators and the other generators of our algebra $\operatorname{are}^{17}$.

$$
\begin{gathered}
{\left[T_{i j}, Q_{m}\right]=\left[P_{+} Q_{i} \delta_{j m}-P_{-} Q_{j} \delta_{i m}\right]} \\
{\left[T_{i j}, Q_{m}^{\prime}\right]=\left[P_{+} Q_{i}^{\prime} \delta_{j m}-P_{-} Q_{j}^{\prime} \delta_{i m}\right]} \\
{\left[T_{i j}, S_{m}\right]=\left[P_{-} S_{i} \delta_{j m}-P_{+} S_{j} \delta_{i m}\right]} \\
{\left[I_{i j}, S_{m}^{\prime}\right]=\left[P_{+} S_{i}^{\prime} \delta_{j m}-P_{-} S_{j}^{\prime} \delta_{i m}\right]} \\
{\left[I_{i j}, M_{p q}\right]=0}
\end{gathered}
$$

Odd elements obey the following anticommutation relations.

$$
\begin{aligned}
&\left\{Q_{i \alpha}, Q_{j \beta}\right\}=(\not P C)_{\alpha \beta} \delta_{i j} \\
&\left\{S_{i \alpha}, S_{j \beta}\right\}=(\not \not C)_{\alpha \beta} \delta_{i j} \\
&\left\{Q_{i \alpha}, S_{j \beta}\right\}= \frac{\delta_{i j}}{2}\left[\left(M_{\mu \nu} \Gamma_{\mu} \Gamma_{\nu} C\right)_{\alpha \beta}+2 C_{\alpha \beta} D\right] \\
&+2(i)\left(P_{+} C\right)_{\alpha \beta} T_{i j}-2(i)\left(P_{-} C\right)_{\alpha \beta} T_{j i} \\
&+(i / 2)\left(C \sigma_{5}\right)_{\alpha \beta} R
\end{aligned}
$$

Note that in our current choice of basis, $Q^{\prime}$ and $S^{\prime}$ transform in inverse representations, that is, a lower index with a tilde is equivalent to a raised

\footnotetext{
${ }^{17}$ see appendix 1 for notation
} 
index with no tilde. This comment should make the index structure of the equation below clearer.

$$
\begin{gathered}
\left\{Q_{i \alpha}^{\prime}, Q_{j \beta}^{\prime}\right\}=\left(H^{\prime} C\right)_{\alpha \beta} \delta_{i j} \\
\left\{S_{i \widetilde{\alpha}}^{\prime}, S_{j \widetilde{\beta}}\right\}=\left(\widetilde{H^{\prime}} C\right)_{\widetilde{\alpha \beta}} \delta_{i j} \\
\left\{Q_{i \alpha}^{\prime}, S_{j \widetilde{\beta}}^{\prime}\right\}=\quad\left((i) \delta_{i j} / 2\right)\left[\left(M_{\mu \nu}^{\prime} \Gamma_{\mu} \Gamma_{\nu}\right)_{\alpha \widetilde{\beta}}+2 \delta_{\alpha \widetilde{\beta}} D^{\prime}\right] \\
-2\left(P_{+}\right)_{\alpha \widetilde{\beta}} T_{i j}+2\left(P_{-}\right)_{\alpha \widetilde{\beta}} T_{j i}+\frac{1}{2}\left(\sigma_{5}\right)_{\alpha \widetilde{\beta}} R \\
\mathbf{d}=\mathbf{5}
\end{gathered}
$$

Two new things happen in five dimensions. Firstly, $S O(4,1)$ does not admit real spinors, and so our $Q$ and $S$ spinors are chosen to be pseudo real, that is they occur in pairs, and obey

$$
\begin{aligned}
Q_{i \alpha} & =\Omega_{i j}\left(C \sigma_{0}^{T}\right)_{\alpha}^{\beta} Q_{j \beta}^{\dagger} \\
S_{i \alpha} & =\Omega_{i j}\left(C \sigma_{0}^{T}\right)_{\alpha}^{\beta} S_{j \beta}^{\dagger}
\end{aligned}
$$

Here $\Omega$ is the $2 n \times 2 n$ pseudoreality matrix consisting of $\mathrm{n}$ diagonal 2 $\times 2$ blocks, each of which is $-i \sigma_{2}$. $\mathrm{C}$ is the charge conjugation matrix $C \Gamma^{T} C^{-1}=\Gamma$, obeying $C^{*}=-C^{-1}, C=-C^{T}$. This implies the following conjugation relation for primed quantities defined in $3.2,3.3$.

$$
\begin{aligned}
& Q_{i \alpha}^{\prime}=\Omega_{i j}\left(C \sigma_{0}^{T}\right)_{\alpha}^{\widetilde{\beta}} S_{j \widetilde{\beta}}^{\prime \dagger} \\
& S_{i \widetilde{\alpha}}^{\prime}=\Omega_{i j}\left(C \sigma_{0}^{T}\right)_{\widetilde{\alpha}}^{\beta} Q_{j \beta}^{\prime \dagger}
\end{aligned}
$$

The second new thing that happens in $d=5$ is that the superconformal algebra exists only for a single pair of $Q s$ (and $S s$ ). This is something we will see a reason for in a section 4 , but it may also be regarded as a fact that will be discovered by anyone who tries to write down an $n \geq 2$ algebra that obeys all Jacobi identities.

The R symmetry for this supersymmetry algebra is $S p(1)=S U(2)$. Denote $\mathrm{R}$ symmetry generators $T_{a}, \mathrm{a}=1, \ldots, 3$. They generate the standard $S U(2)$ algebra. $Q$ s and $S$ s transform as spinors under this $S U(2)$.

$$
\left[T_{a}, Q_{i}\right]=\left(-\sigma_{a}^{\prime} / 2\right)_{i}^{j} Q_{j}
$$




$$
\begin{aligned}
{\left[T_{a}, S_{i}\right] } & =\left(+\sigma_{a}^{\prime} / 2\right)_{i}^{j} S_{j} \\
{\left[T_{a}, Q_{i}^{\prime}\right] } & =\left(-\sigma_{a}^{\prime} / 2\right)_{i}^{j} Q_{j}^{\prime} \\
{\left[T_{a}, S_{i}^{\prime}\right] } & =\left(-\sigma_{a}^{\prime} / 2\right)_{i}^{j} S_{j}^{\prime}
\end{aligned}
$$

$\sigma$ 's above are ordinary Pauli matrices, the primes have been put to distinguish them from $S O(d-1,1) \Gamma$ matrices. ${ }^{18}$

Odd elements anticommute as

$$
\begin{gathered}
\left\{Q_{i \alpha}, Q_{j \beta}\right\}=(\not P C)_{\alpha \beta} \epsilon_{i j} \\
\left\{S_{i \alpha}, S_{j \beta}\right\}=(\not K C)_{\alpha \beta} \epsilon_{i j} \\
\left\{Q_{i \alpha}, S_{j \beta}\right\}=\frac{\delta_{i j}}{2}\left[\left(M_{\mu \nu} \Gamma_{\mu} \Gamma_{\nu} C\right)_{\alpha \beta}+2 C_{\alpha \beta} D\right]-6(i)\left(T_{a} \sigma_{a} / 2\right)_{i j} C_{\alpha \beta}
\end{gathered}
$$

Where we have used the fact that $\mathrm{n}=1$ to replace the $\Omega$ matrix by $\epsilon=$ (i) $\sigma_{2}^{\prime}$, the antisymmetric $2 \times 2$ matrix.

Primed variables obey

$$
\begin{gathered}
\left\{Q_{i \alpha}^{\prime}, Q_{j \beta}^{\prime}\right\}=\left(\not^{\prime} C\right)_{\alpha \beta} \epsilon_{i j} \\
\left\{S_{i \widetilde{\alpha}}^{\prime}, S_{j \widetilde{\beta}}\right\}=\left(\widetilde{\not}{ }^{\prime} C\right)_{\widetilde{\alpha} \widetilde{\beta}} \epsilon_{i j} \\
\left\{Q_{i \alpha}^{\prime}, S_{j \widetilde{\beta}}^{\prime}\right\}=\left[\left(\delta_{i j}(i) / 2\right)\left[\left(M_{\mu \nu}^{\prime} \Gamma_{\mu} \Gamma_{\nu}\right)_{\alpha}^{\theta}+2 \delta_{\alpha}^{\theta} D^{\prime}\right]+6\left(T_{a} \sigma_{a}^{\prime} / 2\right)_{i j} \delta_{\alpha}^{\theta}\right]\left[\left(-\epsilon_{k j} \sigma_{0} C\right)_{\theta \widetilde{\beta}}\right]
\end{gathered}
$$

The last equation implies that

$$
\begin{gathered}
\left\{Q_{i \alpha}^{\prime}, Q^{\prime_{j}^{\dagger}}\right\}=\left(\delta_{i j}(i) / 2\right)\left[\left(M_{\mu \nu} \Gamma_{\mu} \Gamma_{\nu}\right)_{\alpha}^{\theta}+2 \delta_{\alpha}^{\theta} D\right]+6\left(T_{a} \sigma_{a} / 2\right)_{i j} \delta_{\alpha}^{\theta} \\
\mathbf{d}=\mathbf{6}
\end{gathered}
$$

The algebra in $d=6$ is very similar to that in $d=5$ in structure. Once again spinors are pseudo real, that is, obey

$$
Q_{i \alpha}=\Omega_{i j}\left(C \sigma_{0}^{T}\right)_{\alpha}^{\beta} Q_{j \beta}^{\dagger}
$$

\footnotetext{
${ }^{18}$ The minuses in the above equation have been motivated by considerations similar to those that forced us to choose $(+i / 4)\left[\Gamma_{\mu}, \Gamma_{\nu}\right]$ for the Lorentz transformations of the spinors. See the footnote above 3.1
} 


$$
S_{i \alpha}=\Omega_{i j}\left(C \sigma_{0}^{T}\right)_{\alpha}^{\beta} S_{j \beta}^{\dagger}
$$

$\Omega$ is defined at the beginning of the section on $d=5$. In 6 dimensions we have a choice of 2 inequivalent $\mathrm{C}$ matrices to work with. We choose to work with $C \Gamma^{T} C^{-1}=-\Gamma, C^{T}=C, C^{*}=C^{-1}$.

Primed quantities enjoy the following reality properties.

$$
\begin{aligned}
& Q_{i \alpha}^{\prime}=\Omega_{i j}\left(C \sigma_{0}^{T}\right)_{\alpha}^{\widetilde{\beta}} S_{j \widetilde{\beta}}^{\prime \dagger} \\
& S_{i \widetilde{\alpha}}^{\prime}=\Omega_{i j}\left(C \sigma_{0}^{T}\right)_{\widetilde{\alpha}}^{\beta} Q_{j \beta}^{\prime \dagger}
\end{aligned}
$$

It turns out (and we will explain why in the next section) that, although in $d=6$ supercharges of different chiralities are inequivalent (because chiral spinors are pseudoreal not complex, unlike $d=4$ ) superconformal algebras exist only when all $Q s$ have the same chirality. We will choose this to be the positive chirality. Define $P_{+}=\left(1+\sigma_{7}\right) / 2$. Note $P_{+}^{\dagger}=P_{+} ; C^{-1} P_{+} C=P_{-}$. Superconformal algebras exist with any (even) number - $2 \mathrm{n}$ - of $Q \mathrm{~s}$ (paired by $\Omega$ as usual). The R symmetry group is $S p(n)$. Writing out the details of the theory for general $\mathrm{n}$ would involve unfamiliar looking formulae in terms of $S p(n)$ generators. Happily, the cases relevant to physics - $\mathrm{n}=1$ and $\mathrm{n}=2$ -can be written more simply as $S p(1)=S U(2)$, and $S p(2)=S O(5)$. We will list the algebra only for these special cases.

$$
\mathrm{n}=1
$$

The R symmetry group is $S U(2)$; supercharges transform as spinors under this group.

$$
\begin{aligned}
& {\left[T_{a}, Q_{i}\right]=\left(-\sigma_{a}^{\prime} / 2\right)_{i}^{j} Q_{j}} \\
& {\left[T_{a}, S_{i}\right]=\left(+\sigma_{a}^{\prime} / 2\right)_{i}^{j} S_{j}} \\
& {\left[T_{a}, Q_{i}^{\prime}\right]=\left(-\sigma_{a}^{\prime} / 2\right)_{i}^{j} Q_{j}^{\prime}} \\
& {\left[T_{a}, S_{i}^{\prime}\right]=\left(-\sigma_{a}^{\prime} / 2\right)_{i}^{j} S_{j}^{\prime}}
\end{aligned}
$$

Odd elements anticommute thus

$$
\begin{array}{r}
\left\{Q_{i \alpha}, Q_{j \beta}\right\}=\left(P_{+} \not P C\right)_{\alpha \beta} \epsilon_{i j} \\
\left\{S_{i \alpha}, S_{j \beta}\right\}=\left(P_{-} \not \not C\right)_{\alpha \beta} \epsilon_{i j}
\end{array}
$$




$$
\left\{Q_{i \alpha}, S_{j \beta}\right\}=\frac{\delta_{i j}}{2}\left[\left(M_{\mu \nu} P_{+} \Gamma_{\mu} \Gamma_{\nu} C\right)_{\alpha \beta}+2\left(P_{+} C\right)_{\alpha \beta} D\right]-8(i)\left(T_{a} \sigma_{a} / 2\right)_{i j}\left(P_{+} C\right)_{\alpha \beta}
$$

Where $\epsilon$ is the $\Omega$ matrix for $n=1$, defined as for $\mathrm{d}=5$. Primed variables obey

$$
\begin{gathered}
\left\{Q_{i \alpha}^{\prime}, Q_{j \beta}^{\prime}\right\}=\left(P_{+} \not^{\prime} C\right)_{\alpha \beta} \epsilon_{i j} \\
\left\{S_{i \widetilde{\alpha}}^{\prime}, S_{j \widetilde{\beta}}^{\prime}\right\}=\left(P_{+} \widetilde{K}^{\prime} C\right)_{\widetilde{\alpha} \widetilde{\beta}} \epsilon_{i j} \\
\left\{Q_{i \alpha}^{\prime}, S_{j \widetilde{\beta}}^{\prime}\right\}=\left[\left(\delta_{i j}(i) / 2\right)\left[\left(M_{\mu \nu}^{\prime} P_{+} \Gamma_{\mu} \Gamma_{\nu}\right)_{\alpha}^{\theta}+2\left(P_{+}\right)_{\alpha}^{\theta} D^{\prime}\right]\right. \\
\left.+8\left(T_{a} \sigma_{a}^{\prime} / 2\right)_{i j}\left(P_{+}\right)_{\alpha}^{\theta}\right]\left[\left(-\epsilon_{k j} \sigma_{0} C\right)_{\theta \widetilde{\beta}}\right]
\end{gathered}
$$

The last equation implies that

$$
\begin{gathered}
\left.\left\{Q_{i \alpha}^{\prime}, Q_{j}^{\prime \theta \dagger}\right\}=\left(\delta_{i j}(i) / 2\right)\left[\left(M_{\mu \nu}^{\prime} P_{+} \Gamma_{\mu} \Gamma_{\nu}\right)_{\alpha}^{\theta}+2\left(P_{+}\right)_{\alpha}^{\theta} D^{\prime}\right]+8\left(T_{a} \sigma_{a}^{\prime} / 2\right)_{i}^{j}\left(P_{+}\right)_{\alpha}^{\theta}\right] \\
\mathbf{n}=\mathbf{2}
\end{gathered}
$$

The algebra for the $n=2$ case is very similar to that for $n=1$. To obtain it one needs only to make some minor modifications on the $n=1$ algebra. The R symmetry group for $\mathrm{n}=2$ is $S O(5)$, and so one replaces $T_{a}$ in the $\mathrm{n}=1$ algebra with $T_{a b},(S O(5)$ generators $), \mathrm{a}, \mathrm{b}$ run from $1, \ldots, 5 .\left(\sigma^{\prime}\right) / 2$ in the $\mathrm{n}=1$ algebra is replaced by $(1 / 4)(-i / 4)\left[\Gamma_{a}^{\prime}, \Gamma_{b}^{\prime}\right]$. R symmetry $S O(5) \Gamma$ matrices are primed to prevent confusion with Euclidian $S O(d) \Gamma$ matrices. ${ }^{19}$

As an example, the last equation in our section on $n=1$, when adopted to $\mathrm{n}=2$ reads

$$
\begin{aligned}
\left\{Q_{i \alpha}^{\prime}, Q_{j}^{\prime \theta \dagger}\right\}= & \left(\delta_{i j}(i) / 2\right)\left[\left(M_{\mu \nu} P_{+} \Gamma_{\mu} \Gamma_{\nu}\right)_{\alpha}^{\theta}+2\left(P_{+}\right)_{\alpha}^{\theta} D\right] \\
& \left.+(8 / 4)\left(T_{a b}(-i / 4)\left[\Gamma_{a}^{\prime}, \Gamma_{b}^{\prime}\right]\right)_{i}^{j}\left(P_{+}\right)_{\alpha}^{\theta}\right]
\end{aligned}
$$

\footnotetext{
${ }^{19}$ Part of the factor $(1 / 4)$ accounts for the over counting in the range of indices when the generators of $S O(5) \mathrm{R}$ symmetry are contracted with the matrix above. The factor may be checked by noting that the $\mathrm{n}=2$ algebra has $2 \mathrm{n}=1$ sub-algebras (because $S O(5)$ contains $S O(4)=S U(2) \times S U(2))$, and then requiring that the sub-algebras reduce to the $\mathrm{n}=1$ case derived above.
} 
Where it is to be remembered that the $\Gamma^{\prime}$ matrices have nothing to do with spacetime $\Gamma$ matrices.

\subsection{First Unitarity Restrictions}

We now derive level one unitarity restrictions on representations of the superconformal algebra. Some general comments first. As for conformal algebras, our representation will be irreducible unitary modules chosen to be diagonal in the maximal compact subgroup ; Rsym $\times S O(2) \times S O(d)$. States in the module are labeled by $\mathrm{R}$ symmetry weights, a scaling dimension (Eigenvalue of $D^{\prime}$ ) and Lorentz Group weights. As for the conformal group, the set of states with a given dimension (eigenvalue under the $S O(2)$ ) form a finite dimensional, and in general reducible, representation of $S O(d) \times(R$ algera $)$. Weight vectors in a representation of physical interest always have a lowest dimension. States of this lowest dimension $\epsilon_{0}$ host a representation of $S O(d) \times S O(2) \times R$ symmetry that is necessarily irreducible. The lowest (or highest) weights or this finite dimensional irreducible representation label and completely specify the entire module.

Every state in our module may be be obtained by allowing every $Q^{\prime}$ operator to act on the lowest weight state at most once, and then permitting the conformal group operators to act on these $Q \mathrm{~s}$ an indefinite number of times. The superconformal irreducible representation splits into a number of different conformal group irreducible representations whose lowest weight states are all obtained by acting specific combinations of $Q^{\prime}$ s on the Lowest weight state of the entire module.

We now proceed to derive unitarity constraints on the superconformal modules. We perform some calculations at the lowest level - in analogy with the calculations performed for the conformal group.

$$
\mathrm{d}=3
$$

Let our states of lowest dimension be denoted by $|\{t\} m\rangle$ where $\{t\}$ labels $S O(n)$ (R sym ) weights, and $m$ is the half integer that labels weights in the Lorentz $S O(3)$. Unitarity demands the positivity of the matrix

$$
A_{\nu\{t\} m^{\prime} j, \mu\{s\} m i}=<\{t\} m^{\prime}\left|S_{j \nu}^{\prime} Q_{i \mu}^{\prime}\right|\{s\} m>
$$

Using 3.27 and $D^{\prime}=-i \epsilon_{0}$, this implies that all negative eigenvalues of the matrix $B$ have modulus $\leq \epsilon_{0}$, where 


$$
\left.B_{\nu\{t\} m^{\prime} j, \mu\{s\} m i}=\left(i \delta_{i j} / 2\right)\left(M_{a b}\right)_{m^{\prime} m} \Gamma_{a} \Gamma_{b} C\right)_{\mu \nu}-(i) \delta_{\mu \nu}\left(I_{i j}\right)_{\{s\}\{t\}}
$$

Happily $B$ is the sum of two matrices, one of which has only Lorentz indices and the other of which has only $\mathrm{R}$ sym indices, so we may separately diagonalize each piece and add the relevant eigenvalues.

The $\mathrm{R}$ symmetry piece is diagonalized exactly as we diagonalized $M_{i j}$ when dealing with the conformal group. The various eigenvalues of this part of $\mathrm{B} \operatorname{are}^{20}-\frac{1}{2}\left[c_{2}\left(R^{\prime}\right)+c_{2}(R)+c_{2}(\right.$ vector $\left.)\right]$ for various $R^{\prime}$ occurring in the Clebsch Gordan of decomposition of vector $\times R$. For the most negative eigenvalue we pick the $R^{\prime}$ with the largest Casimir. If $\mathrm{R}$ has highest weights $\left(h_{1} \ldots, h_{n}\right)$ then the $R^{\prime}$ with the largest Casimir has highest wt $\left(h_{1}+1, h_{2}, h_{3} \ldots, h_{n}\right)$, and so the most negative eigenvalue is simply $=-h_{1}$ (using 2.40).

The Lorentz part is diagonalized similarly. We recognize the matrix expression as the matrix element of the operator 2J.S where S is a spin operator, and $\mathrm{J}$ is an angular momentum operator in the given representation ${ }^{21}$. The lowest eigenvalue of this operator is 0 for $j=0$, or $-(j+1)$ otherwise. So we have $\mathrm{e}^{22}$

$$
\begin{gathered}
\epsilon_{0} \geq h_{1} \quad(j=0) \\
\epsilon_{0} \geq h_{1}+j+1 \quad(j \neq 0)
\end{gathered}
$$

Where $h_{1}$ is the highest weight of the representation under the $\mathrm{R}$ symmetry group and $j$ the highest weight of the $S O(3)$ Lorentz representation. Note that even for $h_{1}=0$, this is a slightly stronger condition than that obtained from conformal invariance alone [2.42 - 2.44].

For comparison with the next section it is useful to have a list of all values of $\epsilon_{0}$ (not merely the maximum) for which our $A$ matrix has a zero eigen value. These values are

$$
\epsilon_{0}=j+1-\delta_{0 j}+\frac{1}{2}\left(c_{2}\left(R^{\prime}\right)-c_{2}(R)-(n-1)\right)
$$

\footnotetext{
${ }^{20}$ Careful with the order of indices - $I$ appears as a transpose

${ }^{21}$ note $\mathrm{M}$ appears as a transpose, and $-M^{T}$ obeys the same commutation relations as $\mathrm{M}$

${ }^{22}$ These results agree with the special cases worked out for $\mathrm{n}=8, j=0$ by Seiberg, reported in [15], equation 4.1. When comparing result one must be careful to account for the fact that in [15] supercharges were taken to transform in the spinor representation of $S O(8)$, (possible because of triality)
} 


$$
\epsilon_{0}=-j+\frac{1}{2}\left(c_{2}\left(R^{\prime}\right)-c_{2}(R)-(n-1)\right)
$$

Where $R^{\prime}$ runs through all allowed representations. Using appendix 2, we work out all allowed values of $\frac{1}{2}\left(c_{2}\left(R^{\prime}\right)-c_{2}(R)-(n-1)\right)$. They $\operatorname{are}^{23}$ $h_{i}+1-i$ for those values of $\mathrm{i}$ for which $h_{i-1} \neq h_{i}$, and $-h_{i}-n+i+1$ for those values of $i$ for which $h_{i} \neq 0$, so that if we define

$$
\begin{aligned}
c_{i, n}^{1} & =2-i+j-\delta_{0 j}+h_{i} \\
c_{i, n}^{2} & =1-i-j+h_{i} \\
c_{i, n}^{3} & =2-n+i+j-\delta_{0 j}-h_{i} \\
c_{i, n}^{4} & =1-n+i-j-h_{i}
\end{aligned}
$$

we find that our matrix has zero eigenvalues at $\epsilon_{0}$ equal to $c_{i, n}^{1}$ or $c_{i, n}^{2}$ for $i$ such that $h_{i-1} \neq h_{i}$ and $\epsilon_{0}$ equal to $c_{i, n}^{3}$ or $c_{i, n}^{4}$ for i such that $h_{i} \neq 0$.

$$
\mathrm{d}=4
$$

Once again we demand the positivity of

$$
A_{\nu\{t\} m^{\prime} n^{\prime} j, \mu\{s\} m n i}=\left\langle\{t\} m^{\prime} n^{\prime}\left|S_{j \nu}^{\prime} Q_{i \mu}^{\prime}\right|\{s\} m n\right\rangle
$$

$\{s\}$ and $\{t\}$ now stand for $U(n)$ weights, $\left(m, m^{\prime}\right),\left(n, n^{\prime}\right)$ are the " $J_{z}$ " values of our states for the $2 J_{\mathrm{s}}, J_{1}$ and $J_{2}$ that comprise the rotation $S O(4)$. Let $j_{1}$ be the highest wt of the positive chirality angular momentum $J_{1}$ and $j_{2}$ the highest weight of the negative chirality angular momentum $J_{2}$. As usual, we process the RHS of 3.79 using 3.41. The condition, as for $d=3$, is of the form $\epsilon_{0} \geq$ - (smallest eigenvalue of auxiliary matrix). The presence of 2 chiralities introduces a slightly new feature; the auxiliary matrix is a direct sum of two matrices, one associated with +ve chirality, the second with -ve chirality - we call these two matrices the $P_{+}$and $P_{-}$matrices respectively. Recalling that in the transition from $S O(4)$ to $S U(2) \times S U(2)$, $M \cdot M=2\left(\left(J_{1}\right)^{2}+\left(J_{2}\right)^{2}\right)$ and being careful about signs, the $P_{+}$matrix is (in operator form) $4 J_{1} \cdot S_{1}-2 T_{i j} V_{i j}+R / 2$. Here $V_{i j}$ is the fundamental $S U(n)$ matrix generator in the notation of Appendix 1. The $P_{-}$matrix

\footnotetext{
${ }^{23}$ Here, as through the rest of this paper, slight differences arise between the cases $\mathrm{n}$ even and $\mathrm{n}$ odd, for $d=3$. The cases of interest for physics are $\mathrm{n}=1,2,4,8$. The case $\mathrm{n}=1$ is easy and has been explicitly worked out in [10]. Therefore here, and in the rest of the paper we list formulae only for $\mathrm{n}$ even. The corresponding formulae for odd $\mathrm{n}$ are generally slight modifications. Here, for instance, $\mathrm{n}$ odd admits all the values listed plus $-\frac{n-1}{2}$
} 
is $4 J_{2} \cdot S_{2}-2 T_{i j}\left(V^{*}\right)_{i j}+\frac{1}{2} R$, where $V^{*}$ is the $S U(N)$ generator in the antifundamental representation, i.e., $\left[\left(V^{*}\right)_{i j}\right]_{m n}=-\delta_{i n} \delta_{j m}$.

To compute $2 T \cdot V$ we note it is $(T+V)^{2}-T^{2}-V^{2}$, i.e., $C(T \times V)-$ $C(T)-C(V) . T \times V$ stands for all representations that can be obtained from $T$ by tensoring with $V$. If $T$ is characterized by the numbers $\left\{r_{i}\right\}$ (appendix 1), thinking in terms of a Young Tableaux, we see that the only representations that can occur in $T \times V$ are those with $r_{i}$ increased by unity for a single value of $i(=k$ say) and remaining unchanged for all other $i$. The allowed values of $k$ are those for which $R_{k-1} \neq 0$ (appendix 1). When $T \times V$ couple to give $r_{k}^{\prime}=r_{k}+1$ the operator $\left(2 T \cdot V-\frac{1}{2} R\right)$ takes value $\left(2 r_{k}-\frac{2 \Sigma\left(r_{i}\right)}{n}+2-2 k+\frac{R(4-n)}{2 n}\right)$. Computation of $T \cdot V^{*}$ is similar. Coupling with $V^{*}$ corresponds to adding a box to the dual young Tableaux, i.e., removing a box from the Young Tableaux. This corresponds to putting $r_{i}^{\prime}=r_{i}-1$ for some $i=k$ st $R_{k} \neq 0$, and leaving $r_{i}$ unchanged for all other values of $i$. When $T \times V^{*}$ couple to give $r_{k}^{\prime}=r_{k}-1$ the value of $\left(2 T \cdot V^{*}-R / 2\right)$ is $\left(-2 r_{k}+\frac{2 \Sigma\left(r_{i}\right)}{n}-2 n+2 k-\frac{R(4-n)}{2 n}\right)$. Of course $2 J \cdot S$ has the usual values $-(j+1)$ and $j$. Putting this all in we see that our matrix has a zero eigenvalue at

$$
\begin{aligned}
& \epsilon_{0}=d_{n k}^{1}=2 j_{1}+4+2 r_{k}-2 \Sigma\left(r_{i}\right) / n-2 k+\frac{R(4-n)}{2 n}+\left(-2 \delta_{j_{1} 0}\right) \\
& \epsilon_{0}=d_{n k}^{2}=-2 j_{1}+2+2 r_{k}-\frac{2 \Sigma\left(r_{i}\right)}{n}-2 k+\frac{R(4-n)}{2 n}
\end{aligned}
$$

Where $k$ runs over values st $R_{k-1} \neq 0$ in our representation.

$$
\begin{aligned}
& \epsilon_{0}=d_{n k}^{3}=2 j_{2}+2-2 r_{k}+\frac{2 \Sigma\left(r_{i}\right)}{n}+2 k-\frac{R(4-n)}{2 n}-2 n+\left(-2 \delta_{j_{2} 0}\right) \\
& \epsilon_{0}=\quad d_{n k}^{4}=2 j_{2}-2 r_{k}+\frac{2 \Sigma\left(r_{i}\right)}{n}+2 k-\frac{R(4-n)}{2 n}-2 n
\end{aligned}
$$

Where $k$ runs over values st $R_{k} \neq 0$ in our representation.

The unitarity constraint is obtained by requiring that $\epsilon_{0} \geq$ the the largest of these values. Since $d^{1} \geq d^{2}$, and $d^{3} \geq d^{4}$, we want the maximum of $\left(d^{1}, d^{3}\right) . d^{1}$ takes its maximum at $k=1$, always an allowed value. $d^{3}$ takes its maximum at $k=n$, also always an allowed value. Therefore our unitarity constraint is

$$
\epsilon_{0} \geq \max \left(d_{n 1}^{1}, d_{n n}^{3}\right)
$$

This is consistent with, and almost identical to the conditions derived in [3] for $d=4$. We will have more to say about this in the next section. 
$d=5$

The evaluations of level one results in this case is very similar to our evaluation in $d=3$, and I will only present answers, obtained using (3.54). A representation that transforms under the Lorentz Group $S O(5)$ with highest weights $\left(h_{1}, h_{2}\right)$ and under the R symmetry group $S O(3)$ with highest weight ('angular momentum value') $\mathrm{k}$ possesses a scaling dimension that obeys the following inequality.

$$
\epsilon_{0} \geq-\left(c_{2}\left(R^{\prime}\right)-c_{2}(R)-\frac{5}{2}\right)+3 k
$$

where $R$ is the $S O(5)$ representation $\left(h_{1}, h_{2}\right)$, and $R^{\prime}$ is any representation that can be obtained from $R$ by tensoring it with the spinor representation.

The values of scaling dimension at which our ' $A$ ' matrix develops a zero eigenvalue are

$$
\begin{gathered}
\epsilon_{0}=-\left(c_{2}\left(R^{\prime}\right)-c_{2}(R)-\frac{5}{2}\right)+3 k \\
\epsilon_{0}=-\left(c_{2}\left(R^{\prime}\right)-c_{2}(R)-\frac{5}{2}\right)-3 k-3\left(1-\delta_{k 0}\right)
\end{gathered}
$$

for all $R^{\prime}$ s present in the Clebsch Gordan decomposition of $R$ with the spinor representation of $S O(5)$. Using appendix 2, we write an explicit formula for all possible values of $\left(c_{2}\left(R^{\prime}\right)-c_{2}(R)-\frac{5}{2}\right)$.

$$
\begin{gathered}
c_{2}\left(R^{\prime}\right)-c_{2}(R)-\frac{5}{2}=h_{1}+h_{2} \\
c_{2}\left(R^{\prime}\right)-c_{2}(R)-\frac{5}{2}=h_{1}-h_{2}-1 \\
c_{2}\left(R^{\prime}\right)-c_{2}(R)-\frac{5}{2}=-h_{1}+h_{2}-3 \\
c_{2}\left(R^{\prime}\right)-c_{2}\left(R^{\prime}\right)-\frac{5}{2}=-h_{1}-h_{2}-4
\end{gathered}
$$

The restrictions put are (derived from the rules in appendix 2)

a) If $h_{2}=0$ then the 2nd and 4th of these do not occur. b) If $h_{2}=h_{1}=0$ then the 2nd, 3rd and 4th of these do not occur. c) If $h_{2}=h_{1}$ then the 3 rd 
of these does not occur. Thus our matrix develops a zero eigenvalue at $\epsilon_{0}$ equal to

$$
\begin{aligned}
& e^{1}=3 k-h_{1}-h_{2} \\
& e^{2}=-3 k-3\left(1-\delta_{k 0}\right)-h_{1}-h_{2}
\end{aligned}
$$

for all values of $\left(h_{1}, h_{2}\right)$

$$
\begin{aligned}
& e^{3}=3 k-h_{1}+h_{2}+1 \\
& e^{4}=-3 k-3\left(1-\delta_{k 0}\right)-h_{1}+h_{2}+1
\end{aligned}
$$

for $\left(h_{1}, h_{2}\right)$ s.t. $h_{2} \neq 0$

$$
\begin{aligned}
& e^{5}=3 k+h_{1}-h_{2}+3 \\
& e^{6}=-3 k-3\left(1-\delta_{k 0}\right)+h_{1}-h_{2}+3
\end{aligned}
$$

for $\left(h_{1}, h_{2}\right)$ s.t. $h_{1} \neq h_{2}$

$$
\begin{aligned}
& e^{7}=3 k+h_{1}+h_{2}+4 \\
& e^{8}=-3 k-3\left(1-\delta_{k 0}\right)+h_{1}+h_{2}+4
\end{aligned}
$$

for $\left(h_{1}, h_{2}\right)$ s.t. $h_{2} \neq 0$

$$
\mathrm{d}=6
$$

Once again evaluation is simple, and I only present answers obtained using 3.70 and its partner. Our representations transform under the Lorentz Group $S O(6)$ with highest weights $\left(h_{1}, h_{2}, h_{3}\right)$ and, for $\mathrm{n}=1,2$ under the $\mathrm{R}$ symmetry group $S O(3), S O(5)$ with highest weights $k,\left(l_{1}, l_{2}\right)$.

$$
\mathrm{n}=1
$$

The scaling dimension obeys

$$
\epsilon_{0} \geq-\left(c_{2}\left(R^{\prime}\right)-c_{2}(R)-\frac{15}{4}\right)+4 k
$$

where $R$ is the $S O(5)$ representation $\left\{h_{i}\right\}$, and $R^{\prime}$ is any representation present in the Clebsch Gordan decomposition of $R$ with the chiral spinor representation.

The values of scaling dimension at which the ' $A$ ' matrix develops a zero eigenvalue are

$$
\epsilon_{0}=-\left(c_{2}\left(R^{\prime}\right)-c_{2}(R)-\frac{15}{4}\right)+4 k
$$




$$
\epsilon_{0}=-\left(c_{2}\left(R^{\prime}\right)-c_{2}(R)-\frac{15}{4}\right)-4 k-4+4 \delta_{k 0}
$$

with $R^{\prime}$ varying over the $S O(6)$ representations spoken of above. We list all values taken by $-\left(c_{2}\left(R^{\prime}\right)-c_{2}(R)-\frac{15}{4}\right)$ (derived using appendix 2 ).

$$
\begin{gathered}
-\left(c_{2}\left(R^{\prime}\right)-c_{2}(R)-\frac{15}{4}\right)=\left(-h_{1}-h_{2}-h_{3}\right) \\
-\left(c_{2}\left(R^{\prime}\right)-c_{2}(R)-\frac{15}{4}\right)=\left(-h_{1}+h_{2}+h_{3}+2\right) \\
-\left(c_{2}\left(R^{\prime}\right)-c_{2}(R)-\frac{15}{4}\right)=\left(+h_{1}-h_{2}+h_{3}+4\right) \\
-\left(c_{2}\left(R^{\prime}\right)-c_{2}(R)-\frac{15}{4}\right)=\left(+h_{1}+h_{2}-h_{3}+6\right)
\end{gathered}
$$

The first of these always occurs. The second occurs if $h_{2}-\frac{1}{2} \geq\left|h_{3}-\frac{1}{2}\right|$. The third occurs if $h_{1} \neq h_{2}$. The fourth occurs if $h_{2}-\frac{1}{2} \geq\left|h_{3}+\frac{1}{2}\right|$.

Thus our A matrix develops a zero eigenvalue at $\epsilon_{0}$ equal to

$$
\begin{aligned}
& f^{1+}=4 k-h_{1}-h_{2}-h_{3} \\
& f^{1-}=-4 k-4\left(1-\delta_{k 0}\right)-h_{1}-h_{2}-h_{3}
\end{aligned}
$$

for all values of $\left(h_{1}, h_{2}, h_{3}\right)$

$$
\begin{aligned}
& f^{2+}=4 k-h_{1}+h_{2}+h_{3}+2 \\
& f^{2-}=-4 k-4\left(1-\delta_{k 0}\right)-h_{1}+h_{2}++h_{3}+2
\end{aligned}
$$

$$
\begin{aligned}
& \text { for }\left(h_{1}, h_{2}, h_{3}\right) \text { s.t. }\left|h_{3}-\frac{1}{2}\right| \leq h_{2}-\frac{1}{2} \\
& \qquad \begin{aligned}
f^{3+}=4 k+h_{1}-h_{2}+h_{3}+4 \\
f^{3-}=-4 k-4\left(1-\delta_{k 0}\right)+h_{1}-h_{2}+h_{3}+4
\end{aligned}
\end{aligned}
$$

for $\left(h_{1}, h_{2}, h_{3}\right)$ s.t. $h_{1}-h_{2} \geq 1$

$$
\begin{aligned}
& f^{4+}=4 k+h_{1}+h_{2}+-h_{3}+6 \\
& f^{4-}=-4 k-4\left(1-\delta_{k 0}\right)+h_{1}+h_{2}-h_{3}+6
\end{aligned}
$$




$$
\text { for }\left(h_{1}, h_{2}, h_{3}\right) \text { s.t. } h_{2}-\frac{1}{2} \geq \begin{array}{r}
\left|h_{3}+\frac{1}{2}\right| \\
\mathbf{n}=\mathbf{2}
\end{array}
$$

The derived bound on scaling dimensions is

$$
\epsilon_{0} \geq-\left(c_{2}\left(R^{\prime}\right)-c_{2}(R)-\frac{15}{4}\right)+2\left(l_{1}+l_{2}\right)
$$

where $R$ is the $S O(5)$ representation $\left\{h_{i}\right\}$, and $R^{\prime}$ is any representation that can be obtained from $R$ by tensoring it with the chiral spinor representation, the values of the term in the bracket being given by (3.97 - 3.98).

Note that $2\left(l_{1}+l_{2}\right)=4 k_{1}$, where $k_{1}$ and $k_{2}$ are the $S U(2) \times S U(2)$ highest weights in the $S O(4)=S U(2) \times S U(2)$ subgroup of $S O(5)$, and so our $\mathrm{n}=2$ results are consistent with the $\mathrm{n}=1$ results.

The values of scaling dimension at which our ' $A$ ' matrix develops a zero eigenvalue are (subject to restrictions given below)

$$
\begin{array}{r}
\epsilon_{0}=g_{R^{\prime}}^{1}=-\left(c_{2}\left(R^{\prime}\right)-c_{2}(R)-\frac{15}{4}\right)+2\left(l_{1}+l_{2}\right) \\
\epsilon_{0}=g_{R^{\prime}}^{2}=-\left(c_{2}\left(R^{\prime}\right)-c_{2}(R)-\frac{15}{4}\right)+2\left(l_{1}-l_{2}-1\right) \\
\epsilon_{0}=g_{R^{\prime}}^{3}=-\left(c_{2}\left(R^{\prime}\right)-c_{2}(R)-\frac{15}{4}\right)+2\left(-l_{1}+l_{2}-3\right) \\
\epsilon_{0}=g^{4} R^{\prime}=-\left(c_{2}\left(R^{\prime}\right)-c_{2}\left(R^{\prime}\right)-\frac{15}{4}\right)+2\left(-l_{1}-l_{2}-4\right)
\end{array}
$$

The restrictions put are (derived from the rules in appendix 2): a) If $l_{2}=$ 0 then $g_{R^{\prime}}^{2}, g_{R^{\prime}}^{4}$ no longer yield zero eigenvalue dimensions. b) If $l_{2}=l_{1}=0$ then $g_{R^{\prime}}^{2}, g_{R^{\prime}}^{3}, g_{R^{\prime}}^{4}$ no longer yield zero eigenvalue dimensions. c) If $l_{2}=l_{1}$ then $g_{R^{\prime}}^{3}$ no longer yields an acceptable zero eigenvalue dimension.

It would be too tedious to explicitly list all 16 possible values of $\epsilon_{0}$ that result from substituting each of $3.97-3.100$ into each of $3.106-3.109$, but that procedure would generate the list of zero eigenvalue scaling dimensions of our algebra. 


\section{Lie Super Algebras}

The generators of the Superconformal group, like those of Poincare Supersymmetry, belong a lie super algebra (LSA), an algebra consisting of two parts, the even and the odd such that even $\times$ even $=$ even, odd $\times$ odd $=$ even, odd $\times$ even $=$ odd, even $\times$ odd $=$ odd, Here $\times$ refers to the commutator / anticommutator between elements of the LSA as appropriate. Elements of an LSA are also required to satisfy a graded Jacobi identity.

The theory of Lie super algebras has been studied in detail by a number of authors. Simple, finite dimensional, complex Lie Super Algebras have been completely classified. They are of two types: Classical Lie Super Algebras, and Cartan Lie super algebras. We will describe the classification of Classical LSAs in subsection 4.1. In subsection 4.2 we will state a result by Kac on the representation of LSAs, and discuss how this result may be put to use in the task of determining conditions for the unitarity of representations of superconformal algebras. In subsections $4.4-4.7$ we will apply the Kac criterion to the special cases of superconformal algebras in $d=3,4,5,6$ respectively, and complete the task of determining unitarity restrictions on the scaling dimensions of representations of superconformal algebras.

A good general reference for the theory of LSAs is [7] .

\subsection{Classical Lie Super Algebras}

A simple finite dimensional complex LSA G $=G_{0}+G_{1}$ is said to be classical if the representation of the even part, $G_{0}$ on the odd part $G_{1},{ }^{24}$ is completely reducible. ${ }^{25}$ It turns out that the even part of a classical LSA is always a Lie Algebra.

Classical LSA's may be sub classified as those of type 1, those for which the representation of $G_{0}$ on $G_{1}$ is reducible, and those of type 2, those for which the representation of $G_{0}$ on $G_{1}$ is irreducible.

$G_{0}$ for a type 2 LSA turns out always to be a semisimple Lie Algebra. ${ }^{26}$ Below we list all the Classical LSAs of type 2, specifying their names, their

\footnotetext{
${ }^{24}$ the representation is formed by taking the commutator of even with odd elements - it is a representation of $G_{0}$ on $G_{1}$ because the commutator of an even and an odd element is always odd

${ }^{25}$ i.e., can be decomposed into the direct sum of blocks irreducible representations

${ }^{26}$ A Lie Algebra that has no U(1) factors
} 
$G_{0}$ content, and the representation of $G_{0}$ on $G_{1} \cdot{ }^{27}$

$$
\begin{array}{ccc}
G=G_{0}+G_{1} & G_{0} & G_{0} \text { Rep on } G_{1} \\
B(m, n) & B_{m}+C_{n} & \text { vector } \times \text { vector } \\
D(m, n) & D_{m}+C_{n} & \text { vector } \times \text { vector } \\
D(2,1, \alpha) & A_{1}+A_{1}+A_{1} & \text { vector } \times \text { vector } \times \text { vector } . \\
F(4) & B_{3}+A_{1} & \text { spinor } \times \text { vector } \\
G(3) & G_{2}+A_{1} & \text { spinor } \times \text { vector } \\
Q(n) & A_{n} & \text { adjoint }
\end{array}
$$$$
G_{0} \quad \text { Rep on } G_{1}
$$ \\ $G_{0}$ Rep on $G_{1}$ \\ vector $\times$ vector \\ vector $\times$ vector \\ vector $\times$ vector $\times$ vector . \\ spinor $\times$ vector \\ spinor $\times$ vector \\ adjoint
}

Type 1, basic, Classical LSAs turn out to have $G_{0}$ s that are not in general semi simple. These algebras admit a consistent $\mathrm{Z}$ gradation, : i.e., $G_{1}$ splits into into two pieces $G_{1}=G_{\overline{1}}+G_{-1}$ such that $G_{\bar{i}} \times G_{\bar{j}} \subseteq G_{\bar{i}+\bar{j}}{ }^{28}$. The representation of $G_{0}$ on $G_{1}$ is always reducible for type 1 algebras; however it turns out that the representations of $G_{0}$ on $G_{\overline{1}}$ and on $G_{-1}$ are each irreducible and in fact are contragradient ${ }^{29}$. We list all the Basic Classical LSAs of type 1 , specifying their names, their $G_{0}$ content, and the representation of $G_{0}$ on $G_{-1}$.

$$
\begin{array}{ccc}
G=G_{0}+G_{1} & G_{0} & G_{0} \text { Rep on } G_{-1} \\
A(m, n) & A_{m}+A_{n}+C & \text { vector } \times \text { vector } \times C \\
A(m, m) & A_{m}+A_{n} & \text { vector } \times \text { vector } \\
C(n) & C_{n-1}+C & \text { vector } \times C
\end{array}
$$

$\mathrm{C}$ denotes the abelian algebra of complex numbers or its one dimensional representation.

There is exactly one LSA of type one that is not basic. For this algebra, the representation of $G_{0}$ on $G_{-1}$ and its representation on $G_{\overline{1}}$ are not contragradient. This algebra is named $\mathrm{P}(\mathrm{n})$, its even part is the lie algebra $A_{n}$; $A_{n}$ is represented on $G_{\overline{1}}$ as the (antisymmetric tensor)* , and on $G_{\overline{1}}$ as the symmetric tensor.

That completes our listing of the classical LSAs. It will be useful for the rest of the paper to have descriptions of some of these algebras in terms of

\footnotetext{
${ }^{27}$ We use Cartan Notation for Lie Algebras; recall that $A_{n}$ is $S U(n+1), B_{n}$ is $S O(2 n+1)$, $C_{n}$ is $S p(n), D_{n}$ is $S O(2 n)$

${ }^{28}$ Define $G_{\overline{0}}=G_{0} . \times$ as usual here denotes the (anti) commutator

${ }^{29}$ Two representations are said to be contragradient if the set of weights in one representation is minus the set of weights in the other
} 
supermatrices. We provide this description below. See [7], sec 2.1 for more details.

Consider a graded vector space of even dimension $m$ and odd dimension $n$. Operators on this space may be represented as square $(m+n) \times(m+n)$ complex matrices. These matrices possess an $\mathrm{m} \times \mathrm{m}$ diagonal block that maps even vectors to even vectors, and an $\mathrm{n} \times \mathrm{n}$ diagonal block that maps odd vectors onto odd vectors, as well as off diagonal blocks that map odd vectors to even ones and vice versa. We endow these matrices with a $Z_{2}$ grading, defining elements belonging to the diagonal blocks to be even elements, and elements belonging to off diagonal block above to be odd elements. We now convert this space of matrices into an LSA by defining the LSA product $x$ as the commutator / anticommutator of matrices in the usual fashion.

Let the space of all $m \times n$ matrices with the $Z_{2}$ gradation above be called $\mathrm{gl}(\mathrm{m}, \mathrm{n})$. Endow the base $\mathrm{m}+\mathrm{n}$ dimensional vector space ( the space on which our matrices act) with a bilinear form ('scalar product'), denoted below by $\mathrm{F}($, ), that is symmetric on even elements, antisymmetric on odd elements and ensures orthogonality between odd and even elements. ${ }^{30}$

Define the following sub spaces of $\mathrm{gl}(\mathrm{m}, \mathrm{n})$.

$\mathrm{sl}(\mathrm{m}, \mathrm{n})=$ Matrices of zero supertrace belonging to $\mathrm{gl}(\mathrm{m}, \mathrm{n})$.

$\operatorname{osp}(m, n)=$ Matrices $A$ in $g l(m, n)$ s.t. $F(A x, y)=F(x, A y)$ for all $x, y$ in the base vector space.

One may identify some of the Classical basic LSAs described behind with these matrix algebras. ${ }^{31}$

$$
\begin{aligned}
A(m, n) & =\operatorname{sl}(m+1, n+1) \quad(m \neq n \quad m, n \geq 0) \\
A(m, m) & =\operatorname{sl}(m+1, m+1) / I \quad(m \geq 0) \\
B(m, n) & =\operatorname{osp}(2 m+1,2 n) \quad(m \geq 0 \quad n>0) \\
C(n) & =\operatorname{osp}(2,2 n) \quad(n>0) \\
D(m, n) & =\operatorname{ops}(2 m, 2 n)(m \geq 2 ; n \geq 0)
\end{aligned}
$$

$\mathrm{Q}(\mathrm{n})$ and $\mathrm{P}(\mathrm{n})$ may also be represented as matrices.

$\mathrm{Q}(\mathrm{n})(n \geq 2)$ is the sub-algebra of $\mathrm{A}(\mathrm{n}, \mathrm{n})$ which consists of the matrices

$$
\left(\begin{array}{ll}
a & b \\
b & a
\end{array}\right)
$$

\footnotetext{
${ }^{30}$ See [7], Sec 2.1 for a matrix realization of this scalar product.

${ }^{31}$ The case $A(m, m)$ is special below because the $U(1)$ factor in the even part of $\mathrm{sl}(\mathrm{m}+1, \mathrm{n}+1)$ becomes the identity matrix when $\mathrm{m}=\mathrm{n}$, and so $\mathrm{sl}(\mathrm{m}+1, \mathrm{~m}+1)$ is not a simple LSA. One thus has to mod it out by identity to obtain the simple LSA A(m,m).
} 
where $\mathrm{a}$ and $\mathrm{b}$ are $n \times n$ matrices with $\operatorname{tr}(\mathrm{b})=0$.

$\mathrm{P}(\mathrm{n})(n \geq 2)$ is the sub-algebra of $\mathrm{A}(\mathrm{n}, \mathrm{n})$ that consists of matrices

$$
\left(\begin{array}{cc}
a & b \\
c & -a^{T}
\end{array}\right)
$$

where a,b,c are $n \times n$ matrices with $\operatorname{tr}(\mathrm{a})=0$ and $\mathrm{b}=$ symmetric, $\mathrm{c}=$ skew symmetric.

This completes our summary of the classification of simple classical LSAs. Note that the even part of a Cartan LSA cannot be a lie algebra, because all representations of a Lie algebra are reductive.

A couple of definitions we will use ahead. The even roots of a classical LSA are the roots of its even component, $G_{0}$. The odd roots of a classical LSA are the weights of the representation of $G_{0}$ on $G_{1}$.

The algebras that we will identify as superconformal algebras each possess a unique bilinear form, i.e., a map $(,)^{32}: \mathcal{A} \times \mathcal{A} \rightarrow C$, which enjoys the following properties. 1) $(\mathrm{a}, \mathrm{b})=0$ if $\mathrm{a}$ is even and $\mathrm{b}$ is odd or vice versa. 2) $(a, b)=(-)^{n}(b, a)$, where $n=0$ if $a, b$ are even, and $n=1$ if a,b are odd. 3 ) $(\mathrm{a} \times \mathrm{b}, \mathrm{c})=(\mathrm{a}, \mathrm{b} \times \mathrm{c})$. For those superalgebras that have a simple super-matrix realization, a realization of $(\mathrm{a}, \mathrm{b})$ is $\operatorname{str}(a b){ }^{33}$

Finally a comment on positivity. As in Lie algebra theory it is convenient to impose a notion of positivity on the roots of our LSA. Defining positivity is equivalent to specifying a maximal solvable sub-algebra -the sub-algebra of raising operators with positive roots - in our LSA. ${ }^{34}$. All Maximal solvable sub-algebras in a Lie algebra are equivalent under similarity transformations, and so all choices of positivity on a Lie algebra are equivalent. The corresponding result is not true for Classical LSAs. The set of inequivalent conventions of positivity (inequivalent Borel sub-algebras) have been classified and listed in proposition 1.2 in [6] . It will only be be important for us to note that not all conventions of positivity give the same results - we will have to adopt one on physical grounds ahead.

\footnotetext{
${ }^{32}(\mathrm{a}, \mathrm{b})$ will sometimes be written as a.b in the next section

${ }^{33} \mathrm{str}$ denotes the supertrace, i.e. the trace of the upper diagonal block minus the trace of the lower diagonal block of a super-matrix

${ }^{34}$ a sub-algebra is said to be solvable if multiplying the sub-algebra with itself a finite number of times gives zero ; a maximal solvable sub-algebra is called a Borel sub-algebra
} 


\subsection{How Superconformal algebras fit into the classification}

It is now easy to see why no superconformal algebras exist in $d>6$. By a superconformal algebra we mean an algebra whose even part $G_{0}$ possesses as a sub-algebra the conformal algebra $S O(d, 2)$, represented spinorially on the odd part of the algebra, $G_{1}$. Shnider ([8], lemma 1) has shown that if there exists a complex superconformal algebra, then there exists a simple one. But we have a list of all simple classical algebras. ${ }^{35}$ Which classical LSAs have $S O(d)$ sub-algebras? The LSAs B $(m, n)$ and $D(m, n)$ do, but these are always represented vectorially on the odd part of the algebra according to $4.1,4.2$ and so are not superconformal algebras. Other than these, the only $S O(d+2)$ factor explicit in $4.1,4.2$ is in $\mathrm{F}(4)$, which has a $B_{3}=S O(7)$ factor. This is indeed represented spinorially on the odd part. Thus F(4) is a superconformal algebra. From 4.1 we identify the $\mathrm{R}$ symmetry of this superconformal algebra as $A_{1}=S U(2)=S O(3)$. The spinors transform in the vector of $S U(2)$, i.e., the spinor of $S O(3)$. Thus $\mathrm{F}(4)$ is the $d=5$, $n=1$ superconformal algebra we have constructed in the previous section. We now have a 'reason' for why the only superconformal algebra in $d=5$ is $n=1$.

This analysis seems to indicate that there are no superconformal algebras except $n=1$ in $d=5$, but that is not quite right, as we have omitted to account for isomorphisms between low dimensional lie algebras. Recalling that $S O(5)=S p(2)=C_{2}$, and that the spinor representation of the first is the vector representation of the second, we immediately deduce that $\mathrm{B}(\mathrm{m}, 2)$ and $\mathrm{D}(\mathrm{m}, 2)$ are superconformal algebras in $\mathrm{d}=3$, with $\mathrm{R}$ symmetry algebras $B_{m}=S O(2 m+1)$ and $D_{m}=S O(2 m)$ respectively - these are the $d=3, \mathrm{n}=2 \mathrm{~m}+1$ and $\mathrm{n}=2 \mathrm{~m}$ superconformal algebras we have constructed in the previous section. Since $S O(6)=S U(4)$ and that the spinor representation of the first is the vector representation of the second, we deduce that $\mathrm{A}(3, \mathrm{~m})$ is a superconformal algebra in $\mathrm{d}=4$, with $\mathrm{R}$ symmetry $A_{m}+C$, i.e., $S U(m+1) \times U(1)=U(m+1) \cdot{ }^{36}$ These are the $d=4, \mathrm{n}=\mathrm{m}+1$ superconformal algebras we have constructed in the previous section. The case $d=5$ we have dealt with - $S O(7)$ has no isomorphisms, and so there exists only the $\mathrm{n}=1$ algebra $\mathrm{F}(4) . S O(8)$ has no isomorphisms either and so it seems that no $d=6$ superconformal algebra exists. However $S O(8)$ possesses the magical property of triality - its vector and spinor representations are permuted under automorphisms, and so $\mathrm{B}(4, \mathrm{n})$ supplemented by a triality

\footnotetext{
${ }^{35}$ We deal with complex algebras at the moment, so do not distinguish between $S O(d, 2)$ and $S O(d+2)$.

${ }^{36}$ Except when $\mathrm{m}=3$, in which case the $\mathrm{R}$ symmetry may be either $S U(4)$ or $U(4)$, depending on whether the algebra is simple or not
} 
transformation, is the superconformal algebra in $d=6$, with $\mathrm{R}$ symmetry $C_{m}=S p(m)$ corresponding to the $\mathrm{n}=\mathrm{m}$ algebras constructed in the previous section. Note that under a particular triality automorphism, all vectors are mapped to spinors of the a particular chirality. Therefore all $S O(8)$ spinors $\mathrm{V}$ in the $d=6$ superconformal algebra have the same chirality, as in our construction in the previous section. ${ }^{37} S O(m)$ for $m>8$ is not isomorphic to any other Lie algebra, nor is its vector representation equivalent to its spinor representation in any way. Hence we conclude that there exist no superconformal algebras in $d>6$.

\subsection{Kac Theory and Unitarity}

Recall what we know about representations of superconformal algebras. Representations are modules of states, that can be built out of a lowest weight state by successively acting upon it with $Q^{\prime}$ raising operators. The representation is completely determined by the lowest weight state, which in turn is specified by a scaling dimension $\epsilon_{0}$, the weights of an $S O(d)$ representation, and the weights of the R symmetry representation. Group commutation relations completely specify the scalar product between different states in the module, and thus it is not obvious, and indeed not true, that the module will have states with positive norm for all possible lowest weight states.

However states at level $\mathrm{m},{ }^{38}$ have norms that are polynomials in $\epsilon_{0}$ of degree $\leq m$ with positive coefficient for the leading power of $\epsilon_{0}$. Therefore all states have positive norm at large enough $\epsilon_{0}$. Consider a sequence of representations with fixed $\mathrm{R} \operatorname{sym} \times S O(d)$ lowest weights but varying $\epsilon_{0}$. At high enough $\epsilon_{0}$ all states in the module have positive norm. As we decrease $\epsilon_{0}$ towards zero, we eventually hit a value, say $\epsilon_{0}=a$, at which a single state $|\psi\rangle$ and all its descendents attain zero norm. At this value of $\epsilon_{0}$ the module becomes reducible. ${ }^{39}$ As $\epsilon_{0}$ is lowered below $a$, states develop negative norm and the theory becomes non unitary.

If $|\psi\rangle$ is a level one state then its norm as a function of $\epsilon_{0}$ is proportional to $\left(\epsilon_{0}-a\right)$ and that is the end of the story. Unitary representations exist for $\epsilon_{0} \geq a$ and do not exist for $\epsilon_{0}\langle a$. If $\mid \psi\rangle$ is a level 2 state then its norm is proportional to $\left(\epsilon_{0}-a\right)\left(\epsilon_{0}-b\right)$. As $\epsilon_{0}$ is lowered below $b$, the set of states generated from $|\psi\rangle$ regain positive norm, and so these states provide

\footnotetext{
${ }^{37} \mathrm{An} S O(8)$ spinor of positive chirality is composed of $2 S O(6)$ spinors, one of positive chirality - $Q$ - and one of negative chirality - $S$

${ }^{38}$ states obtained from the lowest weight state by the application of $\mathrm{m} Q^{\prime}$ operators

${ }^{39}$ because the set of states modulo zero norm states provide a representation for the algebra, - as in a gauge theory, where physical states represent the Lorentz algebra
} 
no obstruction to unitarity for $\epsilon_{0} \leq b$, though other states might. Again at exactly $\epsilon_{0}=b$ the representation becomes reducible. If $|\psi\rangle$ is a level 3 state then its norm is $K\left(\epsilon_{0}-a\right)\left(\epsilon_{0}-b\right)\left(\epsilon_{0}-c\right)$, and the positivity of this norm leaves open a 'window' $[c, b]$ of values of $\epsilon_{0}$ that could lead to unitary representations ... etc., etc.,.

The discussion in the paragraph above is useful for the following reason Kac ([6], sec4, theorem 1, part 1 ) has derived a remarkably simple criterion for a module representation of a lie superalgebra to become reducible (i.e., develop a zero norm state ). His theorem, adopted to the case of a lowest weight module (he used highest weights) states $A$ module with lowest weight $\Lambda$ is reducible if and only if $(\Lambda, \rho)=(\Lambda, \alpha)$ where $\alpha$ is an odd root of the algebra with zero norm, (, ) is the non-degenerate bilinear form on the algebra introduced in the previous section, and $\rho$ is the difference between the half sum of positive even roots and the half sum of positive odd roots of the algebra.

In the rest of this paper we apply this criterion to the representations of $\mathrm{d}=3,4,5,6$ superalgebras, and determine the values $\left(\mathrm{a}, \mathrm{b}, \mathrm{c} .\right.$. ) of $\epsilon_{0}$ that (for fixed other weights specifying the lowest weight state) give rise to reducible representations, i.e., zero norm states.

From the discussion above we see that requiring $\epsilon_{0} \geq \max (a, b, c \ldots)$ is sufficient to guarantee unitarity of representations. If, additionally, the zero norm state that forms as $\epsilon_{0}=$ this maximum is a level one state then as this is also a sufficient condition for unitarity. This will turn out to be the case for large enough representations of the Lorentz $S O(d)$ labeling our lowest weight states. For small representations of the Lorentz $S O(d)$ algebra (eg $j=0$ in $d=3$ ), it will turn out that the state whose norm goes to zero at the $\epsilon_{0}=\max (a, b, c \ldots)$ is not a level one state. In these cases additional argument and calculations will be employed to almost completely determine necessary conditions for unitarity.

\section{$4.4 \mathrm{~d}=3$}

The $d=3, n=n$ superconformal algebra is identified with $\operatorname{osp}(n, 4)$, and so is composed of matrices of the form

$$
\left(\begin{array}{cc}
n \times n & n \times 4 \\
4 \times n & 4 \times 4
\end{array}\right)
$$

Diagonal blocks contain even elements, the off diagonal blocks the odd ones. The even part of our algebra is $S O(n) \times S O(5)$. The conformal group lives 
in the $4 \times 4$ and the $S 0(n) \mathrm{R}$ symmetry group in the $n \times n$. In the workout below we will assume that $n$ is even - the case $n$ odd may be worked out analogously.

Conformal generators are identified within the $4 \times 4$ block as follows (see $2.16-2.19$ for notation).

$$
S_{a b}=\frac{1}{2}\left(\begin{array}{cc}
0 & 0 . \\
0 & -\left(S_{a b}^{s p}\right)^{T}
\end{array}\right)
$$

Here the $4 \times 4$ matrices $S^{s p}$ are $S O(3,2)$ spinor matrices created from the $\Gamma$ matrices given at the beginning of section 2 .

Define

$$
C^{\prime}=\left(\begin{array}{cc}
0 & C \\
C & 0
\end{array}\right)
$$

where $\mathrm{C}$ is the $2 \times 2$ charge conjugation matrix for $S O(2,1)$, defined in the $\mathrm{d}=3$ part of section 3. $C^{\prime}$ obeys $C^{\prime-1} \Gamma C^{\prime}=\Gamma^{T}$ and $C^{\prime T}=-C^{\prime}$. Define $e_{\alpha}^{i}$ as the matrix with one in the $(N+\alpha)^{t h}$ row, and $i^{t h}$ column, and zero everywhere else. Odd elements in the $\mathrm{d}=3$ superconformal algebra are identified with matrices as

$$
V_{i \alpha}=e_{\alpha}^{i}+i C_{\alpha \theta}\left(e_{\theta}^{i}\right)^{T}
$$

Where $\mathrm{V}$ is defined at the beginning of section 2 .

Finally, $S 0(n)$ generators are given by standard vector representation generators of $S O(n)$ in the $\mathrm{n} \times \mathrm{n}$ block, i.e., $\left(I_{i j}\right)_{a b}=(-i)\left(\delta_{i a} \delta_{j b}-\delta_{i b} \delta_{j a}\right)$

One may check that the matrices defined above obey the (anti) commutation relations of the $d=3$ superconformal algebra. As usual, we are interested not in the generators themselves, but in their primed counterparts $2.20-2.23,3.2,3.3)$. The matrices corresponding to these operators may now be constructed from their definitions in the equations just quoted.

Primed operators take on a simpler form in a new basis. ${ }^{40}$

In this basis the two diagonal primed operators are (in the $4 \times 4$ block.)

$$
i D^{\prime}=\frac{1}{2}\left(\begin{array}{cc}
I & 0 \\
0 & -I
\end{array}\right)
$$

\footnotetext{
${ }^{40}$ i.e., on performing a similarity transformation on our osp $(\mathrm{n}, 4)$ matrices, $M^{\prime}=$ $A^{-1} M A$, where $\mathrm{A}$ is a matrix with identity in the $N \times N$ block, zero off diagonal pieces, and matrix $\mathrm{B}$ in the $4 \times 4$ block, with $\mathrm{B}$ given by
}

$$
B=\frac{1}{\sqrt{2}}\left(\begin{array}{cc}
1 & -i \sigma_{0} \\
1 & i \sigma_{0}
\end{array}\right)
$$




$$
M_{23}^{\prime}=\left(\begin{array}{cc}
-\sigma_{3} & 0 \\
0 & \sigma_{3}
\end{array}\right)
$$

The matrices $I_{12}^{\prime}, I_{34}^{\prime} \cdots$ are made diagonal by the standard $S O(n)$ basis transformation, to give $I_{12}^{\prime}=\operatorname{diag}(1,-1,0,0, .$.$) etc.. D^{\prime}, M_{23}^{\prime}$ and these Cartan Is above constitute Cartan sub algebra (CSA) of our algebra. Linear functionals on this Cartan sub algebra can always be written as the operation of multiplying the CSA element with a diagonal matrix (of the CSA form) and taking supertrace With this understanding we can treat diagonal matrices as linear functionals on $\mathrm{H}$, the Cartan sub algebra. Define $e_{i}$ as the diagonal super-matrix with one in the $i^{\text {th }}(i \leq n)$ entry, and zero elsewhere. Define $f_{i}$ $(i \leq 4)$ as the diagonal super-matrix with one in the $(n+i)^{t h}$ entry, and zero elsewhere. Note $\left(e_{i}, e_{j}\right)=\delta_{i j},\left(f_{i}, f_{j}\right)=-\delta_{i j}$. Define $\alpha_{i}=\left(e_{2 i-1}-e_{2 i}\right) / 2$; $\beta_{D}=-\left(f_{1}+f_{2}-f_{3}-f_{4}\right) / 2$ and $\beta_{J}=-\left(-f_{1}+f_{2}+f_{3}-f_{4}\right) / 2$. Note that $\left(\alpha_{i}, \alpha_{j}\right)=\frac{1}{2} \delta_{i j}$ and $\left(\beta_{i}, \beta_{j}\right)=-\delta_{i j} .{ }^{41}$

The even roots of our algebra (the roots of the even part $G_{0}$ ) are those of the conformal algebra $\pm \beta_{D} \pm \beta_{J}, \pm \beta_{D}, \pm \beta_{J}$ (eight in all), and those connected with the R symmetry $\left( \pm \alpha_{i} \pm \alpha_{j}\right)$ The odd roots are $\left( \pm \frac{1}{2} \beta_{D} \pm\right.$ $\left.\frac{1}{2} \beta_{J} \pm \alpha_{i}\right)$

Simple positive roots, may be chosen as

$$
\begin{gathered}
r_{1}=\beta_{D}-\beta_{J} \\
r_{2}=\beta_{J} \\
r_{3}=\beta_{D} / 2-\beta_{J} / 2-\alpha_{1} \\
t_{1}=\alpha_{1}-\alpha_{2}
\end{gathered}
$$

and so on until

$$
t_{\frac{n}{2}-1}=\alpha_{n / 2-1}-\alpha_{n / 2}
$$

This choice of positivity is partly dictated by the requirement that all positive roots, acting on $\mathrm{D}$, yield a positive number. It corresponds intuitively to the ordering $\beta_{D} \geq \beta_{J} \geq \alpha_{1} \ldots \geq \alpha_{n / 2}$. The half sum of even positive

\footnotetext{
${ }^{41}$ We have chosen the definitions of $\alpha_{i}$ etc., such that, $\left(\alpha_{i}, H_{j}\right)=\delta_{i j}$ etc., (the right hand side is always one or zero). Therefore $\beta_{D}$ is a weight vector with positive unit $i D^{\prime}$ weight, and no weight under any other Cartan Generator, etc..
} 
roots, and odd positive roots $\rho_{0}$, and $\rho_{1}$ are given by

$$
\begin{gathered}
\rho_{0}=\frac{3}{2} \beta_{D}+\frac{1}{2} \beta_{J}+\sum_{i=1}^{n / 2} \frac{1}{2} \alpha_{i}(n-2 i) \\
\rho_{1}=\frac{1}{2} n \beta_{D} \\
\rho=\rho_{0}-\rho_{1}=\frac{(3-n)}{2} \beta_{D}+\frac{1}{2} \beta_{J}+\frac{1}{2} \sum_{i=1}^{n / 2} \alpha_{i}(n-2 i)
\end{gathered}
$$

One may now take the inner product of $\rho$ with odd positive roots. Use

$$
\begin{gathered}
\left(\frac{1}{2} \beta_{J}+\frac{1}{2} \beta_{D}\right) \cdot \rho=-1+\frac{n}{4} \\
\left(\frac{1}{2} \beta_{D}-\frac{1}{2} \beta_{J}\right) \cdot \rho=-\frac{1}{2}+\frac{n}{4} \\
\alpha_{i} \cdot \rho=\frac{(n-2 i)}{4}
\end{gathered}
$$

The odd positive roots of zero norm are $\frac{1}{2} \beta_{D} \pm \frac{1}{2} \beta_{J} \pm \alpha_{i}$

If our lowest weight multiplet has dimension $\epsilon_{0}$ and lowest $S O(n)$ wts $=\left(-h_{1},-h_{2},-h_{3}-h_{n / 2}\right)$, and has $S O(3)$ lowest wt $=-j$, then the lowest weight in the module is

$$
\Lambda=-h_{1} \alpha_{1}-h_{2} \alpha_{2}-\ldots-h_{n / 2} \alpha_{n / 2}+d \beta_{D}-j \beta_{j}
$$

The reducibility conditions are $(\Lambda$, root $)=(\rho$, root $)$. These conditions, for the odd positive zero norm roots above are

$$
\begin{aligned}
& c_{i, n}^{\prime 1}=2-i+j+h_{i} \\
& c_{i, n}^{\prime 2}=1-i-j+h_{i} \\
& c_{i, n}^{\prime 3}=2-n+i+j-h_{i} \\
& c_{i, n}^{\prime 4}=1-n+i-j-h_{i}
\end{aligned}
$$


These are precisely the conditions 3.79 without the restrictions on the range of $j$, and without delta function exceptions for the case $j=0$.

The full unitarity restrictions for $d=3$ are now easy to work out. For $j \neq 0$ the largest $c^{\prime}$ is $c_{1, n}^{\prime 1}=c_{1, n}^{1}=1+j+h_{1}$. Since a level one state attains zero norm at this dimension, we conclude that $\epsilon_{0} \geq 1+j+h_{1}$ is the necessary and sufficient condition for unitarity of our module. For $j=0 \mathrm{a}$ level one state does not attain zero norm at the largest $c^{\prime}$, but does at the second largest $c^{\prime}\left(c_{1, n}^{\prime 2}=c_{1, n}^{1}\right)$. Explicit calculation that we do not reproduce here shows that at level 2 there is a state with norm $\left(\epsilon_{0}-c_{1, n}^{\prime 1}\right)\left(\epsilon_{0}-c_{2, n}^{\prime 1}\right)$. Therefore unitarity allows only $\epsilon_{0} \geq 1+h_{1}$ or $\epsilon_{0}=h_{1}$ (remember $j=0$ ). Actually more work is needed in principle to show that the isolated value of $\epsilon_{0}$ is allowed at all levels, but we circumvent that procedure by noting that, independently, for $j=0$ we have very good reason to believe a unitary representation at this value of $\epsilon_{0}$ does exist. ${ }^{42}$

The calculations above have been presented for the case $n=$ even, but the generalization to $\mathrm{n}=$ odd is is a simple matter. However we do not even need to perform this generalization; the only odd $n$ of interest to us is $n=1$ and this special case is rather simple, and has been explicitly worked out (by hand) in a nice paper by Heidenreich [10] - I list his answers here - unitary representations are obtained for $\epsilon_{0} \geq \frac{1}{2}$ if $j=0$, and $\epsilon_{0} \geq j+1$ for $j>0$. Inspection shows that this is condition 2.58 for $j=0$ and condition 3.75 for $j>0$.

\section{$4.5 \mathrm{~d}=4$}

This subsection is included in this paper only for completeness, everything in this subsection may be found in $[3,4,5]$.

$\mathrm{A}(3, \mathrm{n}-1)$ consists of matrices of the form

$$
\left(\begin{array}{cc}
4 \times 4 & 4 \times n \\
n \times 4 & n \times n
\end{array}\right)
$$

Diagonal blocks even elements, the off diagonal blocks are odd ones. The conformal group basically lives in the $4 \times 4$ and the $S U(n)$ R symmetry group in the $n \times n$. The U(1) lives in both diagonal blocks. Specifically, conformal generators are identified within the $4 \times 4$ block as follows.

$$
J_{i}^{\prime 2}=\frac{1}{2}\left(\begin{array}{cc}
0 & 0 . \\
0 & \sigma_{i} .
\end{array}\right)
$$

\footnotetext{
${ }^{42}$ See, for instance [15], section 4
} 


$$
\begin{aligned}
J_{i}^{\prime 1} & =\frac{1}{2}\left(\begin{array}{cc}
\sigma_{i} & 0 . \\
0 & 0
\end{array}\right) \\
i D^{\prime} & =\frac{1}{2}\left(\begin{array}{cc}
I & 0 . \\
0 & -I
\end{array}\right) \\
P_{\mu}^{\prime} & =\left(\begin{array}{cc}
0 & \sigma_{\mu} \\
0 & 0
\end{array}\right) \\
K_{\mu}^{\prime} & =\left(\begin{array}{cc}
0 & 0 \\
\sigma_{\mu} & 0
\end{array}\right)
\end{aligned}
$$

Where we have defined $\sigma_{\mu}=\left(I, \sigma_{i}\right)$ Define also $e_{\alpha}^{i}$ as the matrix with 1 in the $\alpha^{\text {th }}$ row and $(4+i)^{\text {th }}$ column and zeroes everywhere else. Further define $f_{\alpha}^{i}$ as the matrix with 1 in the $(2+\alpha)^{t h}$ row and $(4+i)^{t h}$ column. We make identification of odd elements as

$$
\begin{gathered}
Q_{i \alpha}^{\prime \dagger}=e_{\alpha}^{i} \\
Q_{i \alpha}^{\prime}=\left(f_{\alpha}^{i}\right)^{T} \\
S_{i \alpha}^{\prime}=\epsilon_{\alpha \beta} f_{\beta}^{i} \\
S_{i \alpha}^{\prime \dagger}=\epsilon_{\alpha \theta}\left(e_{\theta}^{i}\right)^{T}
\end{gathered}
$$

Finally, R charge and $S U(n)$ generators are identified as

$$
\begin{gathered}
R=\frac{1}{\frac{4}{n}-1}\left(\begin{array}{cc}
I & 0 \\
0 & \frac{4 I}{n}
\end{array}\right) \\
T_{a}=\left(\begin{array}{cc}
0 & 0 \\
0 & -T_{a}^{T}
\end{array}\right)
\end{gathered}
$$

(The matrices above are $(4+n) \times(4+n))$

The Cartan Sub Algebra of our matrix algebra consists of the set of supertraceless diagonal matrices. Linear functionals on the CSA can always be written as the operation of multiplying the CSA element with a diagonal ${ }^{43}$ matrix and taking supertrace. Define the matrices $e_{i}(i=1, \ldots, 4)$ and $f_{j}$ $(j=1, \ldots, n)$ as in the previous subsection. The even roots of our algebra are $\left(e_{i}-e_{j}\right)$ and $\left(f_{i}-f_{j}\right)$. The odd roots of our algebra are $\left(e_{i}+f_{i}\right)$.

\footnotetext{
${ }^{43}$ and supertraceless for uniqueness - this will not be important for us we will never use uniqueness
} 
Choose a positivity convention that ensures that the state of lowest weight in our module has also the lowest scaling dimension in the module (as we require physically) i.e., a positivity convention that ensures that positive roots acting on $D^{\prime}$, give a positive number. We choose as simple positive roots

$$
\begin{aligned}
\gamma_{1} & =\left(e_{1}-e_{2}\right) \\
\gamma_{2} & =\left(e_{4}-e_{3}\right) \\
\gamma_{3} & =\left(e_{2}+f_{1}\right) \\
\gamma_{4} & =\left(-e_{4}-f_{N}\right) \\
\gamma_{4+k} & =\left(-f_{k}+f_{k+1}\right)
\end{aligned}
$$

This leads to positivity rules for roots with dictionary ordering and $e_{1} \geq$ $e_{2} \geq-e_{3} \geq-e_{4} \geq-f 1 \geq-f_{2} \geq \ldots \geq-f_{N}$.

The half sum of even positive roots, $\rho_{0}$ and the half sum of odd positive roots $\rho_{1}$ and their difference $\rho=\rho_{0}-\rho_{1}$ are.

$$
\begin{gathered}
\rho_{0}=\frac{1}{2}\left[\sum_{i=1}^{n}(n+1-2 i) f_{i}\right]+\frac{1}{2}\left(3 e_{1}+e_{2}-3 e_{3}-e_{4}\right) \\
\rho_{1}=\frac{n}{2}\left[e_{1}+e_{2}-e_{3}-e_{4}\right] \\
\rho=\frac{3-n}{2} e_{1}+\frac{1-n}{2} e_{2}+\frac{n-3}{2} e_{3}+\frac{n-1}{2} e_{4}-\frac{1}{2} \sum_{i=1}^{n}(n+1-2 i) f_{i}
\end{gathered}
$$

One may now compute the dot product of the odd positive roots with $\rho$.

$$
\begin{gathered}
\left(e_{1}+f_{i}\right) \cdot \rho=2-i \\
\left(e_{2}+f_{i}\right) \cdot \rho=1-i \\
\left(-e_{3}-f_{i}\right) \cdot \rho=-(n-1-i) \\
\left(-e_{2}-f_{i}\right) \cdot \rho=-(n-2 i)
\end{gathered}
$$


The lowest weight state in our module may be written as

$$
\begin{aligned}
\Lambda= & \frac{\epsilon_{0}}{2}\left(e_{1}+e_{2}-e_{3}-e_{4}\right)-j_{1}\left(e_{1}-e_{2}\right)+j_{2}\left(e_{3}-e_{4}\right) \\
& +\frac{(4-n)}{n} \frac{R}{8}\left(e_{1}+e_{2}+e_{3}+e_{4}-f_{1}-f_{2}-f_{3}-\ldots-f_{N}\right)+\left(-\sum_{i=1}^{k} f_{i}\right) r_{k}
\end{aligned}
$$

In the equation above, $j_{1}$ and $j_{2}$ are the (half) integers that label the 2 $S U(2) \mathrm{s}$ in the Lorentz group. ${ }^{44}$

The reducibility condition is $(\Lambda-\rho) \cdot \alpha=0$ for some odd root $\alpha$ which has zero norm (i.e., $\alpha \cdot \alpha=0$ ). All odd roots of this algebra have zero norm. Using 4.29 -4.32 and equation 4.34, a representation is reducible iff

$$
\begin{gathered}
\epsilon_{0}=d_{n j}^{\prime 1}=2 j_{1}+4+2 r_{j}-\frac{2 \Sigma\left(r_{i}\right)}{n}-2 j+\frac{R(4-n)}{2 n} \\
\epsilon_{0}=d_{n j}^{\prime 2}=-2 j_{1}+2+2 r_{j}-\frac{2 \Sigma\left(r_{i}\right)}{n}-2 j+\frac{R(4-n)}{2 n} \\
\epsilon_{0}=d_{n j}^{\prime 3}=2 j_{2}+2-2 r_{j}+\frac{2 \Sigma\left(r_{i}\right)}{n}+2 j-\frac{R(4-n)}{2 n}-2 n \\
\epsilon_{0}=d_{n j}^{\prime 4}=2 j_{2}-2 r_{j}+\frac{2 \Sigma\left(r_{i}\right)}{n}+2 j-\frac{R(4-n)}{2 n}-2 n
\end{gathered}
$$

Where $j$ runs from 1 to $n$.

These are exactly equations $3.80-3.81$ without the restrictions on the range of $\mathrm{j}$ and without the special provisos for the cases $j_{1}=0$ or $j_{2}=0$.

Unitarity conditions for $d=4$ follow. A sufficient condition for unitarity is $\epsilon_{0} \geq \max \left(d_{n j}^{\prime 1}, d_{n j}^{\prime 2}, d_{n j}^{\prime 3}, d_{n j}^{\prime 4}\right)$. Unless either $j_{1}=0$ or $j_{2}=0$, the objects $d^{\prime}$ in $4.34-4.37$ are identical to the quantities $d$ in 3.80 and 3.81, and so (by

\footnotetext{
${ }^{44}$ The signs in our parameterization above are slightly subtle. For inst, $J_{2}^{3}$, the z component of $J_{2}$ is a negative member of the Cartan Sub Algebra (because $e_{3}-e_{4}$ is negative), and so the lowest weight state in a $J_{2}$ multiplet is the state with $m=+j_{2}$. Therefore we have chosen our parameterization above such that $\Lambda\left(J_{2}^{3}\right)=j_{2}$. On the other hand $J_{1}^{3}$ is a positive member of the Cartan sub-algebra, and so the state with the lowest weight in a $J_{1}^{3}$ multiplet is one with $m=-j_{1}$ and so our parameterization has $\Lambda\left(J_{1}^{3}\right)=-j_{1}$. The R charge and $i D^{\prime}$ are what they are (have a single given value in the lowest weight sector), and so $\Lambda(D)=\epsilon_{0}, \Lambda(R)=R$ with no funny minus signs. The sign behind $r_{k}$ is similarly deduced.
} 
now familiar logic) this condition is both necessary and sufficient condition for positivity of norm in our module.

If, on the other hand, either $j_{1}=0$ or $j_{2}=0$ then $d \leq d^{\prime}$ and the necessary condition for norm positivity, 3.83 , is potentially weaker than the sufficient condition above (obtained, notice, by replacing $d$ with $d^{\prime}$ in 3.83 ). Actually, if $j_{1}=0 ; j_{2} \neq 0$ and $d_{n n}^{\prime 3} \geq d_{n 1}^{\prime 1}$, or if $j_{1} \neq 0 ; j_{2}=0$ and $d_{n n}^{\prime 3} \leq d_{n 1}^{\prime 1}$ then once again necessary and sufficient conditions coincide, both being given by 3.83 .

However, if $j_{1}=0 ; j_{2} \neq 0$ and $d_{n n}^{\prime 3} \leq d_{n 1}^{\prime 1}$, then the necessary condition for positivity of norm is $\epsilon_{0} \geq \max \left(d_{n 1}^{1}=d_{n 1}^{\prime 2}, d_{n n}^{\prime 3}\right)$ and the sufficient condition $\epsilon_{0} \geq d_{n 1}^{\prime 1}$ - these do not coincide. So there is a window of values of $\epsilon_{0}$, i.e., the interval $\left.\left[\max \left(d_{n 1}^{\prime 2}, d_{n n}^{\prime 3}\right), d_{n 1}^{\prime 1}\right)\right]$, within which the representation may or may not be unitary, consistent with our derivations so far. Explicit calculations [5] , show that a state with norm proportional to $\left(\epsilon_{0}-d_{n 1}^{\prime 1}\right)\left(\epsilon_{0}-d_{n 2}^{\prime 2}\right)$ occurs at level 2. This says that if $d_{n n}^{\prime 3}>d_{n 1}^{\prime 2}$, then no value in the window above is permitted by unitarity. However if $d_{n n}^{\prime 3} \leq d_{n 1}^{\prime 2}$, then the isolated value $\epsilon_{0}=$ $d_{n 1}^{\prime 2}$ is not ruled out by any calculation yet reported. Further calculations reported in the reference just cited confirm that in this case $\epsilon_{0}=d_{n 1}^{\prime 2}$ is indeed an allowed value. Thus in this case the unitarity restriction is then that $\epsilon_{0}$ obey $\epsilon_{0} \geq d_{n 1}^{1}$ or that $\epsilon_{0}=d_{n 1}^{\prime 2}$. Unitarity constraints for the case $j_{2}=0$ are derived analogously. All final results are listed in section 5 .

\section{$4.6 \quad \mathrm{~d}=5$}

Working out the reducibility conditions for the superconformal algebra in $d=5$, though simple in principle, is slightly complicated by the fact that $\mathrm{F}(4)$ has no simple matrix representation, and so (, ) cannot simply be implemented as a supertrace. Actually an explicit form for $($,$) is known$ - it is the killing form of the algebra ( see [9] ) but it seems a bit pointless to go through the bother of performing this evaluation, as experience with the easier cases - $d=3,4,6$ - shows how the reducibility criteria are related to the dimensions at which level one states attain zero norm. I therefore simply conjecture that representations of the $\mathrm{d}=5$ superconformal algebra are reducible iff $\epsilon_{0}$ is one of

$$
\begin{aligned}
e^{\prime 1} & =3 k-h_{1}-h_{2} \\
e^{\prime 2} & =-3 k-3-h_{1}-h_{2} \\
e^{\prime 3} & =3 k-h_{1}+h_{2}+1 \\
e^{\prime 4} & =-3 k-3-h_{1}+h_{2}+1 \\
e^{\prime 5} & =3 k+h_{1}-h_{2}+3
\end{aligned}
$$




$$
\begin{aligned}
e^{\prime 6} & =-3 k-3+h_{1}-h_{2}+3 \\
e^{\prime 7} & =3 k+h_{1}+h_{2}+4 \\
e^{\prime 8} & =-3 k-3+h_{1}+h_{2}+4
\end{aligned}
$$

With this assumption we now go on to deduce unitarity bounds for $\mathrm{d}=5$. Consider Lorentz representations such that

a) $h_{2}>$ zero . Examples of such representations are the spinor, the antisymmetric tensor, and the Rarita Schwinger representation. In this case the largest $e^{\prime} ; e^{\prime 7}$ is $=e^{7}$, i.e., a dimension at which a level one state attains zero norm. Therefore representations are unitary if and only if $\epsilon_{0} \geq$ $3 k+h_{1}+h_{2}+4$. The bounds are $3 k+5,3 k+6$ and $3 k+6$ for the three examples above.

b) $h_{2}=0, \quad h_{1} \neq 0$. Examples of such representations are the vector and the symmetric traceless tensor. The two largest $e^{\prime}$ values are $e^{\prime 7}$ and $e^{15}$ in that order. No state attains zero norm at $\epsilon_{0}=e^{17}$ at level one (see the condition below 3.93), but that does happen at $\epsilon_{0}=e^{15}=e^{5}$ (see the condition below 3.92). Thus this case is similar to the $j=0$ case for $d=3$, that is it is sufficient that $\epsilon_{0} \geq 3 k+h_{1}+4$ for unitarity, but the isolated value $\epsilon_{0}=3 k+h_{1}+3$ may also be permitted by unitarity. No other value of $\epsilon_{0}$ could possibly be allowed. We have performed some calculations at levels higher than one and have not managed to rule out this possibility and so conjecture that this isolated value of $\epsilon_{0}$ indeed does permit a unitary representation. The isolated value occurs at dimension $\left(3 k+4+h_{1}\right)$ and $\left(3 k+5+h_{1}\right)$ for the two examples cited above. The 'continuum' of allowed values begins at $\left(3 k+5+h_{1}\right)$ and $\left(3 k+6+h_{1}\right)$ for these two examples.

c) $h_{1}=0, \quad h_{2}=0$. This is the (Lorentz) scalar representation. The four largest $e^{\prime}$ values are $(3 k+4),(3 k+3),(3 k+1), 3 k$. Only the last of these is a dimension at which a state attains zero norm at level 1 . On general grounds the first window between $(3 k+3)$ and $(3 k+4)$ does not permit unitary representations except possibly at the lower bound. I have diagonalized the equivalent of matrix $\mathrm{A}$ in 3.72 at level 2 (but for the case $\mathrm{d}=5$, and with 2 Qs and $2 Q^{\dagger} s$ sandwiched between states). At this level there appears a state with norm $=\left(\epsilon_{0}-(3 k+3)\right)\left(\epsilon_{0}-3 k\right)$. This rules out all values of $\epsilon_{0}<3 k+3$ other than possibly $\epsilon_{0}=3 k$. Therefore the only possible values scaling dimension permitted by unitarity are $\epsilon_{0} \geq 3 k+4$ and possibly $\epsilon_{0}=3 k, \epsilon_{0}=3 k+3$. No norm calculation I have performed has produced a result that forbids these isolated values : I conjecture that unitary representations exist at these two isolated values of $\epsilon_{0}$. Proving this conjecture would require one to check the norms of states at all levels (upto 8) (a task I have not the energy for), or the use of some clever trick. 


\section{$4.7 \quad \mathrm{~d}=6$}

The $d=6, \mathrm{n}=1 ; 2$ superconformal algebra is identified with $\operatorname{osp}(8,2 ; 4)$, and so is composed of matrices of the form

$$
\left(\begin{array}{ll}
8 \times 8 & 8 \times x \\
x \times 8 & x \times x
\end{array}\right)
$$

Where $\mathrm{x}=2,4$ for $\mathrm{n}=1,2$.

Diagonal blocks contain the even elements, the off diagonal blocks the odd ones. The even part of our algebra is $S O(8) \times S O(2) ; S O(5)$. The conformal group lives in the $8 \times 8$ and the $S O(3) ; S O(5) \mathrm{R}$ symmetry group in the $x \times x$.

Conformal generators are identified within the $8 \times 8$ block as follows (see (2.16 - 2.19) for notation).

$$
S_{a b}=\frac{1}{2}\left(\begin{array}{cc}
-\left(S_{a b}^{s p}\right)^{T} & 0 \\
0 & 0
\end{array}\right)
$$

Here the $8 \times 8$ matrices $S^{s p}$ are the positive chirality projections of $S O(3,2)$ spinor matrices created from the $\Gamma$ matrices given at the beginning of section 2.

$S 0(3) ; S O(5)$ generators are given by the negative transpose of their spinor representations in the $x \times x$ block.

The odd elements of the algebra may be identified with off diagonal matrices, as in the case of $\mathrm{d}=3$. Define $e_{\alpha}^{i}$ as the matrix with one in the $(\alpha)^{t h}$ row, and $(8+i)^{t h}$ column, and zero everywhere else. Odd elements in the $d=6$ superconformal algebra are identified with matrices as

$$
V_{i \alpha}=A C_{i j}^{\prime \prime} e_{\alpha}^{j}+B C_{\alpha \theta}^{\prime}\left(e_{\theta}^{i}\right)^{T}
$$

Where $\mathrm{V}$ is defined at the beginning of section $2, C^{\prime}$ and $C^{\prime \prime}$ are the charge conjugation matrices appropriate to $S O(6,2)$ and $S O(3) ; S O(5)$ spinor representations respectively and $\mathrm{A}$ and $\mathrm{B}$ are constants whose value does not concern us.

One now determines primed operators using their definitions. On making the the basis change that block diagonalizes $i D^{\prime}$ and $M_{a b}^{\prime}$ we obtain that $S O(8)$ Cartan algebra elements are represented within the $8 \times 8$ block by the matrices

$$
M_{12}^{\prime}=\frac{1}{2} \operatorname{diag}(-1,-1,+1,+1,-1,-1,+1,+1)
$$




$$
\begin{aligned}
M_{34}^{\prime} & =\frac{1}{2} \operatorname{diag}(-1,+1,-1,+1,-1,+1,-1,+1) \\
M_{56}^{\prime} & =\frac{1}{2} \operatorname{diag}(-1,+1,+1,-1,+1,-1,-1,+1) \\
i D^{\prime} & =\frac{1}{2} \operatorname{diag}(+1,+1,+1,+1,-1,1,-1,-1)
\end{aligned}
$$

In the case $n=1$, the CSA of the superalgebra is spanned by the 4 elements above and

$$
T_{3}=-\sigma_{3} / 2
$$

within the $2 \times 2$. In the case $n=2$, the CSA of the superalgebra is spanned by the 4 elements above and the following matrices within the $4 \times 4$

$$
\begin{aligned}
& T_{12}=\frac{1}{2} \operatorname{diag}(-1,-1,+1,+1) \\
& T_{34}=\frac{1}{2} \operatorname{diag}(-1,+1,-1,+1)
\end{aligned}
$$

Linear functionals on the CSA can be written as diagonal matrices as behind; define $e_{i}(\mathrm{i}=1 . .8)$ and $f_{j}(\mathrm{j}=1 . . \mathrm{x})$ as in the $d=3$ workout. Define

$$
\begin{aligned}
\alpha_{4} & =\frac{1}{4}\left(-e_{1}-e_{2}+e_{3}+e_{4}-e_{5}-e_{6}+e_{7}+e_{8}\right) \\
\alpha_{3} & =\frac{1}{4}\left(-e_{1}+e_{2}-e_{3}+e_{4}-e_{5}+e_{6}-e_{7}+e_{8}\right) \\
\alpha_{2} & =\frac{1}{4}\left(-e_{1}+e_{2}+e_{3}-e_{4}+e_{5}-e_{6}-e_{7}+e_{8}\right) \\
\alpha_{D} & =\frac{1}{4}\left(+e_{1}+e_{2}+e_{3}+e_{4}-e_{5}-e_{6}-e_{7}-e_{8}\right)
\end{aligned}
$$

In the $\mathrm{n}=1$ case we also define

$$
\beta=f_{1}-f_{2}
$$

In the $\mathrm{n}=2$ case we define

$$
\begin{aligned}
& \beta_{1}=\frac{1}{2}\left(f_{1}+f_{2}-f_{3}-f_{4}\right) \\
& \beta_{2}=\frac{1}{2}\left(f_{1}-f_{2}+f_{3}-f_{4}\right)
\end{aligned}
$$

The definitions above have been chosen such that, for instance, $\alpha_{4}\left(M_{12}^{\prime}\right)=$ $1, \alpha_{4}($ any other element of CSA $)=0$, etc.. $\operatorname{Note}\left(\alpha_{i} \cdot \alpha_{j}\right)=\frac{1}{2} \delta_{i j}$ and that 
$\alpha s$ are orthogonal to $\beta s$. Note also $(\beta, \beta)=-2$ (relevant to $\mathrm{n}=1$ ) and $\left(\beta_{i}, \beta_{j}\right)=-\delta_{i j}($ relevant to $\mathrm{n}=2)$.

The even roots of our algebra (the roots of the even part $G_{0}$ ) are those of the conformal algebra $\pm \alpha_{i} \pm \alpha_{j}$, (24 in all), and those connected with the $\mathrm{R}$ symmetry $\pm \beta$ for $\mathrm{n}=1$, and $\pm \beta_{1} \pm \beta_{2} ; \pm \beta_{1} ; \pm \beta_{2}$ for $\mathrm{n}=2$. The odd roots for $\mathrm{n}=1$ are $\pm \frac{1}{2} \alpha_{D} \pm \frac{1}{2} \alpha_{1} \pm \frac{1}{2} \alpha_{3} \pm \frac{1}{2} \alpha_{4} \pm \frac{1}{2} \beta$, (16 in all; the number of plus signs behind $\alpha s$ is restricted to be even) and for $\mathrm{n}=2\left( \pm \frac{1}{2} \alpha_{D} \pm \frac{1}{2} \alpha_{1} \pm \frac{1}{2} \alpha_{3} \pm\right.$ $\frac{1}{2} \alpha_{4} \pm \frac{1}{2} \beta_{1} \pm \frac{1}{2} \beta_{2}$, (32 in all; the number of plus signs behind $\alpha s$ is restricted to be even)

We choose a set of simple positive roots that enforces positivity rules that correspond to dictionary ordering with $\alpha_{D}>\alpha_{1}>\alpha_{2}>\alpha_{3}>\beta$ for $\mathrm{n}=1$ and $\alpha_{D}>\alpha_{1}>\alpha_{2}>\alpha_{3}>\beta_{1}>\beta_{2}$ for $\mathrm{n}=2$. The half sum of even positive roots, and odd positive roots $\rho_{0}$, and $\rho_{1}$ are given by $(\mathrm{n}=1)$

$$
\begin{gathered}
\rho_{0}=3 \alpha_{D}+2 \alpha_{1}+\alpha_{2}+\frac{1}{2} \beta \\
\rho_{1}=2 \alpha_{D} \\
\rho=\rho_{0}-\rho_{1}=\alpha_{D}+2 \alpha_{1}+\alpha_{2}+\frac{1}{2} \beta
\end{gathered}
$$

and, for $n=2$

$$
\begin{gathered}
\rho_{0}=3 \alpha_{D}+2 \alpha_{1}+\alpha_{2}+\frac{3}{2} \beta_{1}+\frac{1}{2} \beta_{2} \\
\rho_{1}=4 \alpha_{D} \\
\rho=\rho_{0}-\rho_{1}=-\alpha_{D}+2 \alpha_{1}+\alpha_{2}+\frac{3}{2} \beta_{1}+\frac{1}{2} \beta_{2}
\end{gathered}
$$

Let the lowest weight multiplet have dimension $\epsilon_{0}$, lowest $S O(8)$ wts $\left(-h_{1},-h_{2},+h_{3}\right)^{45}$ and $S O(3) ; S O(5)$ lowest wts $=-\mathrm{j}, ;\left(-l_{1},-l_{2}\right)$. The lowest weight in the module is then $(n=1)$

$$
\Lambda=\epsilon_{0} \alpha_{D}-h_{1} \alpha_{1}-h_{2} \alpha_{2}+h_{3} \alpha_{3}-k \beta
$$

and $(n=2)$

$$
\Lambda=\epsilon_{0} \alpha_{D}-h_{1} \alpha_{1}-h_{2} \alpha_{2}+h_{3} \alpha_{3}-l_{1} \beta_{1}-l_{2} \beta_{2}
$$

\footnotetext{
${ }^{45}$ Note that the lowest weights in our module have $h_{3}$ appearing with a positive sign. We have chosen this strange looking convention to ensure that the representation has highest weights $\left(h_{1}, h_{2}, h_{3}\right)$ as was the case in our work out in section 3 . For instance, a chiral spinor that has highest weights $\left(\frac{1}{2}, \frac{1}{2}, \frac{1}{2}\right)$, but lowest weights $\left(-\frac{1}{2},-\frac{1}{2}, \frac{1}{2}\right)$.
} 
The reducibility conditions are $(\Lambda$, root $)=(\rho$, root $)$. These conditions, for the odd positive zero norm roots are $(\mathrm{n}=1) \epsilon_{0}$ is one of

$$
\begin{aligned}
& f^{\prime 1 \pm}=-h_{1}-h_{2}-h_{3}-2+ \pm(-4 k-2) \\
& f^{\prime 2 \pm}=-h_{1}+h_{2}+h_{3}+ \pm(-4 k-2) \\
& f^{\prime 3 \pm}=+h_{1}-h_{2}+h_{3}+2+ \pm(-4 k-2) \\
& f^{\prime 4 \pm}=+h_{1}+h_{2}-h_{3}+4+ \pm(-4 k-2)
\end{aligned}
$$

These are exactly $3.101-3.104$ without the delta function provisos and the restrictions in those equations.

The reducibility conditions for $\mathrm{n}=2$ are $\epsilon_{0}$ is one of

$$
\begin{aligned}
& f^{\prime 1 \pm \pm}=-h_{1}-h_{2}-h_{3}-4+ \pm\left(-2 l_{1}-3\right)+ \pm\left(-2 l_{2}-1\right) \\
& f^{\prime 2 \pm \pm}=-h_{1}+h_{2}+h_{3}-2+ \pm\left(-2 l_{1}-3\right)+ \pm\left(-2 l_{2}-1\right) \\
& f^{\prime 3 \pm \pm}=+h_{1}-h_{2}+h_{3}+ \pm\left(-2 l_{1}-3\right) \pm\left(-2 l_{2}-1\right) \\
& f^{\prime 4 \pm \pm}=+h_{1}+h_{2}-h_{3}+2+ \pm\left(-2 l_{1}-3\right)+ \pm\left(-2 l_{2}-1\right)
\end{aligned}
$$

These are precisely the conditions that follow from (3.106 - 3.109)and (3.97 - 3.100) without the restrictions on applicability present in those equations.

We now analyze the conditions under which unitary representations of these algebras exist. We perform the analysis for the case $n=1$; the $n=2$ case is extremely similar. All results are listed explicitly in the section 6 .

a) $h_{2}-\frac{1}{2} \geq\left|h_{3}+\frac{1}{2}\right|$. Examples of such representations are the antichiral spinor, the antisymmetric tensor, the antichiral Rarita Schwinger representation and the antiselfdual antisymmetric 3 tensor. In this case the largest $f^{\prime} ; f^{\prime 4--}$ is a dimension at which a level one state attains zero norm. Therefore representations are unitary if and only if $\epsilon_{0} \geq 4 k+h_{1}+h_{2}-h_{3}+6$. The bounds are $4 k+\mathrm{y}$, where $\mathrm{y}=7.5,8,8.5$ and 9 for the four examples above.

b) $h_{1}-h_{2} \geq 1$ and the condition of part a) is not met. Examples of such representations are the vector, the symmetric traceless tensor and the chiral Rarita Schwinger representation. The two largest $f^{\prime}$ values are $f^{\prime 4--}$ and $f^{\prime 3--}$ in that order. No state attains zero norm at $\epsilon_{0}=f^{\prime 4--}$ at level one (see below 3.100), but that does happen at $\epsilon_{0}=e^{\prime 3--}=e^{3--}$ (see below 3.99). Thus this case is similar to the $j=0$ case for $d=3$. It is sufficient that $\epsilon_{0} \geq 4 k+h_{1}+h_{2}-h_{3}+6$ for unitarity, but the isolated value $\epsilon_{0}=4 k+h_{1}-h_{2}+h_{3}+4$ may also be permitted by unitarity. No other value of $\epsilon_{0}$ could possibly be allowed. We have performed some calculations 
at levels higher than one and have not managed to rule out this possibility and so conjecture that this isolated value of $\epsilon_{0}$ indeed does permit a unitary representation. Isolated values occur at $\epsilon_{0}=4 k+y$ where $\mathrm{y}=5,6,5.5$ for the 3 examples above. The continuum of allowed values begins at $y=7,8$, 7.5 in these examples.

c) $h_{1}=h_{2}=h_{3}=h \neq 0$. Examples are the chiral spinor, and the selfdual antisymmetric 3 tensor. In this case a level one state attains zero norm only at the $3 r d$ largest of the $f^{\prime} s$, i.e., at $f^{\prime 2--}=2+h+4 k=r($ def $n)$. The largest $f^{\prime}$ is $f^{\prime 4--}=6+h+4 k$, a value we define as $t$, and the second largest is $f^{\prime 3--}=4+h+4 k=s($ def $n)$. On general grounds, unitarity permits representations with $\epsilon_{0} \geq t$. Unitarity forbids representations with $\epsilon_{0}<r$, and with $\epsilon_{0}$ in the range $(s, t)$. The calculations I have performed at levels higher than one have not managed to rule out the window $[r, s]$ - However, in the next section I will present an explicit construction of unitary representations with $\epsilon_{0}=r$ and $\epsilon_{0}=s$. So in summary, short unitary representations exist for $\epsilon_{0}=t, r, s$. Unitary representations also exist for $\epsilon_{0}>t$. Unitary representations with scaling dimensions in the range $(r, s)$ may or may not exist ${ }^{46}$.

d) $h_{1}=h_{2}=h_{3}=0$. This is the Lorentz scalar representation. The four largest $f^{\prime}$ values are $(4 k+6),(4 k+4),(4 k+2), 4 k$. Only the last of these is a dimension at which a state attains zero norm at level one. As usual the first window $(4 k+4,4 k+6)$ does not permit unitary representations. Explicit calculations at level 2 show the existence of a state with norm proportional to $\left(\epsilon_{0}-(4 k+2)\right)\left(\epsilon_{0}-4 k\right)$. Therefore the only allowed values of scaling dimension are $\epsilon_{0} \geq 4 k+6$ and possibly $\epsilon_{0}$ in the interval $[4 k+2,4 k+4]$ and the isolated value $\epsilon_{0}=4 k$.In the next section I present a construction of short representations with scaling dimension $\epsilon_{0}=4 k, 4 k+2,4 k+4$. The calculations I have performed at levels higher than one have not managed to rule out the window $(4 k+2,4 k+4)$. So in summary, short unitary representations exist for $\epsilon_{0}=4 k, 4 k+2,4 k+4,4 k+6$. Unitary representations do exist for $\epsilon_{0}>4 k+6$ and may or may not exist for $\epsilon_{0}$ in the interval $(4 k+2,4 k+4)^{47}$.

\footnotetext{
${ }^{46}$ It may be a relevant observation that at $k=0,2+h=s$ is precisely the scaling dimension of the free conformal scalar field with $h_{1}=h_{2}=h_{3}=h$ as derived in section 2 . This fact perhaps hints that unitary representations exist in the full interval $[4 k+2,4 k+4]$

${ }^{47}$ It may be a relevant observation that at $k=0,2$ is precisely the scaling dimension of the free conformal scalar field as derived in section 2 . This fact perhaps hints that unitary representations exist in the full interval $[4 k+2,4 k+4]$
} 


\section{$5 \quad$ Examples and Applications}

We are interested primarily in the unitary representations of superconformal algebras on the space of local operators in a superconformally invariant quantum field theory. Three such theories are the (intrinsic) theories on the world volumes of the $M_{2}, M_{5}$ and $D_{3}$ brane. In the next three subsections we review what is known about some of the primary operators of these theories and see how this fits with the results obtained in previous sections. In subsections 5.4 and 5.5 I proceed to explicitly construct all the short representations of the $d=6$ algebras whose existence was left open in the previous section. The construction uses the oscillator method.

\subsection{The World Volume Theory of the $M_{2}$ brane}

Consider the $d=3 N=8$ superconformal field theory on the world volume of a single $M_{2}$ brane. The only matter multiplet with $N=8$ supersymmetry has 8 scalars and 8 fermions. The scalars are taken to transform in the vector of $S O(8)$. The fermions transform in the antichiral spinor of $S O(8)$. Supersymmetry generators transform in the chiral spinor of $S O(8) .{ }^{48}$. The Supersymmetry transformation laws are

$$
\begin{gathered}
\delta \lambda^{\widetilde{\alpha}}=\frac{i}{2} \gamma^{\mu} \Gamma_{\alpha \widetilde{\alpha}}^{a} \epsilon^{\alpha} \partial_{\mu} \phi^{a} \\
\delta \phi^{a}=\bar{\epsilon}^{a} \Gamma_{\alpha \widetilde{\alpha}}^{a} \lambda^{\tilde{\alpha}}
\end{gathered}
$$

Each of the $\phi$ and $\lambda$ fields is a conformal primary operator in the theory on the world volume of an $M_{2}$ brane; these operators form part of a single representation of the superconformal group. The superconformal primary operators labeling this representations are the $\phi s$; they transform in the scalar of $S O(3)$, the vector of $S O(8)$ and have scaling dimension $\frac{1}{2}$. Making the triality transformation to return to the conventions of the previous section, this representation transforms in the scalar of $S O(3)$, the chiral spinor of $S O(8)$ and has dimension $\frac{1}{2}$. This representation is the 'remarkable' or singleton representation of $\mathrm{SO}(3,2 / 8)$, and is an example of the isolated $d=3$ representation with $\epsilon_{0}=h_{1}=\frac{1}{2}$, whose existence was predicted in section 4 .

\footnotetext{
${ }^{48}$ In previous sections susy generators transformed in the vector of $S O(8)$. This convention is related to the convention of this section by a triality transformation. We must be careful to take this fact into account when applying the results of the previous sections to this field theory
} 
Consider, now, products of $n$ scalar fields. Such products are also primary operators under the superconformal algebra, and generate the symmetric tensor product of $n$ singleton representations. These representations are labeled $S O(3)$ scalar; $S O(8)(n, 0,0, . .0)$, or after triality $S O(8)\left(\frac{n}{2}, \frac{n}{2}, \ldots \frac{n}{2}\right)$; $\epsilon_{0}=\frac{n}{2}$. These representations are examples of the isolated $d=3$ representations with $\epsilon_{0}=h_{1}=\frac{n}{2} 49$

The theory on the world volume of $m$ coincident $M_{2}$ branes possesses $m^{2}$ $N=8$ singleton supermultiplets ${ }^{50}$. The theory is associated with the gauge group $U(m)$, and unlike the theory of a single $M_{2}$ brane is an interacting superconformal field theory. One may form gauge invariant products of $\phi$ fields by taking the trace of the products of $\phi s$ as $U(m)$ matrices. Such gauge invariant products of $\phi$ fields for $n \geq 2$ are primary operators under the superconformal group. These operators have the same $S O(3) \times \mathrm{SO}(8) \times D$ labels [15] as simple products of $\phi$ fields in the theory of a single $M_{2}$ brane, and therefore generate the same representations of the superconformal algebra.

\section{$5.2 \mathrm{~d}=4 \mathrm{~N}=4$ SYM- The World Volume theory of the 3 brane.}

Consider $N=4, d=4 U(n)$ Super Yang Mills. The microscopic fields of the theory constitute a $U(n)$ adjoint multiplet containing 6 real scalars transforming in the vector of $S O(6)$, a vector gauge boson which is an $S O(6)$ scalar, 4 complex chiral spinors which are chiral $S O(6)$ spinors.

Under supersymmetry these fields transform as ${ }^{51}$

$$
\begin{gathered}
\delta A_{\mu}=i \epsilon_{i} \sigma_{\mu} \bar{\lambda}^{i}-\lambda_{i} \sigma_{\mu} \bar{\epsilon}^{i} \\
\delta M_{i j}=\epsilon_{i} \lambda_{j}-\epsilon_{j} \lambda_{i}+\epsilon_{i j k l} \bar{\epsilon}^{k} \bar{\lambda}^{l} \\
\delta \lambda_{i}=-\frac{1}{2} i \sigma^{\mu \nu} \epsilon_{i} F_{\mu \nu}+2 i \sigma^{\mu} \partial_{\mu} M_{i j} \bar{\epsilon}+2 i\left[M_{i j}, M^{j k}\right] \epsilon_{k}
\end{gathered}
$$

\footnotetext{
${ }^{49}$ These representations have been explicitly constructed by the oscillator method in [17].

${ }^{50}$ a single multiplet in the adjoint of $U(m)$.

${ }^{51}$ Spinors are written in 2 component notation. $\lambda$ denotes a chiral spinor, and $\bar{\lambda}$ denotes its hermitian conjugate which is antichiral. By the product of 2 spinors we mean $\theta \lambda$ we mean $\theta_{\alpha} \lambda_{\beta} \epsilon^{\alpha \beta}$ where $\epsilon^{12}=1$ The R symmetry has been displayed as $S U(4)$. ij are $S U(4)$ fundamental (vector) indices.
} 
The microscopic fields of our theory lie in a supermultiplet under the superconformal algebra- this is the short multiplet with $j_{1}=j_{2}=0 \epsilon_{0}=1$ and $S O(6)$ rep $=(1,0,0){ }^{52}$. The scalar fields $M_{i j}$ are the superconformal primary operators for this multiplet - their scaling dimension is the dimension appropriate to free scalar fields in $d=4$.

As in the previous subsection we may identify the trace of symmetric products of $p M$ fields as primary superconformal operators that transform in the representation $j_{1}=j_{2}=0 ; \epsilon_{0}=p ; S O(6)$ rep $=(\mathrm{p}, 0,0)$, i.e. $S U(4)$ Dynkin labels $=(0, \mathrm{p}, 0)^{53}$

These are examples of short representations of the $d=4, n=4$ algebra. To see this note that this $S U(4)$ Dynkin labeling corresponds to Young Tableaux with $r_{1}=r_{2}=p, r_{3}=0$, where $r_{i}$ is the number of boxes in $i^{\text {th }}$ row of the Dynkin diagram. For the representation under consideration $d_{44}^{\prime 1}=d_{41}^{\prime 3}=p+2 ; d_{41}^{\prime 2}=d_{44}^{\prime 4}=p$. Since $j_{1}=j_{2}=0$ and $d_{41}^{2}=d_{44}^{4}$ there exist a special short representation at $\epsilon_{0}=d_{41}^{2}=p^{54}$, which is exactly the physical case.

\subsection{The world volume theory of the $M_{5}$ brane}

Consider a $d=6 N=2$ superconformal field theory. The only matter multiplet has 6 scalars $\phi^{a}$ transforming as an $S O(6)$ vector, a single self dual two form field $B_{\mu \nu}$, and 4 chiral spinors $\lambda$, transforming under $S O(6)$ as a chiral spinor. The free theory (ie the theory of a single $M_{5}$ brane in the decoupling limit $l_{p}-->0$ ) has only one such matter multiplet (an explicit action may be found in section 4 of [22] for instance). The supersymmetry transformation properties of this theory are given in [22] .

Each of the $\phi, H$ and $\lambda$ fields in the theory on the world volume of an $M_{5}$ brane is a primary operator of the conformal group; these operators form part of a single representation of the superconformal algebra. The primary operators for this representation are the $\phi s$. The representation labels are $S O(6)$ scalar; $S O(5)$ vector $; \epsilon_{0}=2$.

The theory on the world volume of $m$ coincident $M_{5}$ branes possesses $m^{2} N=8$ singleton supermultiplets ${ }^{55}$. The theory is associated with the gauge group $U(m)$. Gauge invariant products of $\phi$ fields are the traces

\footnotetext{
${ }^{52}$ Note however that the operators in this representation for $n>1$ are not gauge invariant

${ }^{53}$ These representations have been explicitly constructed by the oscillator method in [18].

${ }^{54}$ See the full listing of results in the next section

${ }^{55}$ a single multiplet in the adjoint of $U(m)$.
} 
of the products of $\phi \mathrm{s}$ as $U(m)$ matrices. These symmetric products are superconformal primary operators with labels $S O(6)$ scalar; $S O(5)(n, 0)$ $; \epsilon_{0}=2 n$. These are examples of scalar representations with $h_{1}=h_{2}=$ $h_{3}=0, \epsilon_{0}=2\left(l_{1}+l_{2}\right)$, whose existence we were unsure of in the previous section. Apparently these representations exist as short representations of the superconformal algebra.

To establish the existence of the other short representations of the $d=$ $6, n=2$ algebra, in the next two subsections we review the oscillator construction of this algebra given in [19] .

\subsection{Oscillator Constructions of Supergroups}

I now describe the oscillator method for the construction of superalgebras and some of their unitary representations. This method was developed by Gunaydin and collaborators (see the references in [17] , [18] ).

Notice that it is extremely easy to make an explicit unitary construction of a $U(N)$ Lie algebra using free bosonic or fermionic oscillators. The simplest construction of this sort takes the form $T_{j}^{i}=a^{i} a_{j} .{ }^{56}$

An extension of this construction consists of replacing $a^{i} a_{j}$ by $a^{i} a_{j}+$ $b^{i} b_{j}+\ldots$ where $b \ldots$ are independent oscillators. This extension seems trivial, but it enlarges the space on which we work, and so permits the construction of more general representations of the group, as we will see.

To make an oscillator construction of an arbitrary group, one searches for a unitary (compact) subgroup; constructs the lie algebra of that unitary group using oscillators as outlined above, and then attempts to construct the lie algebra of the rest of the group using the same oscillators. Similarly for Lie supergroups. We will present an oscillator construction for the $(\mathrm{N}=2, \mathrm{~d}=6)$ superconformal algebra in the next subsection.

\subsection{The Oscillator Construction of the $\mathrm{d}=6, \mathrm{~N}=2$ algebra}

Much of this subsection is contained in [19], and is reworked here for the convenience of the reader.

\footnotetext{
${ }^{56}$ We use the notation of appendix $1 . T_{j}^{i}$ is the element of the complexified lie algebra corresponding to the $N \times N$ matrix with unity in the $i^{t} h$ row, $j^{t} h$ column, and zeroes everywhere else. Elements of the real Lie Algebra of $U(N)$ are $T_{j}^{i}+T_{i}^{j}$ and $i\left(T_{j}^{i}-T_{i}^{j}\right)$. The upper index $=U(N)$ fundamental; lower index $=U(N)$ antifundamental ; $a^{i}=a_{i}^{\dagger}$, $a_{i}=$ bosonic / fermionic oscillator.
} 
The even part of $S O(6,2 / 2)$ is $S O(6,2) \times S O(5)$. It is natural to implement the $S O(3,2)$ with bosonic oscillators and the $S O(5)$ with fermionic oscillators. We proceed to do so.

The maximal compact subgroup of $S O(6,2)$ is $S O(6) \times S O(2)=U(4)$. Using the philosophy of sec 2.2 , this compact part may be thought of as consisting of Euclidianized Lorentz transformations and dilatations : the remainder of the group consists of momentum and special conformal transformations. These are vectors under $S O(6)$ but have weight \pm 1 under $U(1)$ dilatations, and hence are respectively antisymmetric tensors of the fundamental / antifundamental representations of $U(4)$. The construction of this group with $n=2 p^{57}$ oscillators is

$$
\begin{gathered}
T_{j}^{i}=a^{i} a_{j}+b_{j} b^{i} \\
S_{i j}=a_{i} b_{j}-a_{j} b_{i} \\
S^{i j}=a^{i} b^{j}-a^{j} b^{i}
\end{gathered}
$$

Here we have suppressed an internal 'which oscillator' index on $a^{\prime} s$ and $b^{\prime} s$. There are $p$ varieties of $a$ and $b$ oscillators, a summation over the $p$ oscillator flavours is implied in all formulae unless otherwise specified. We identify $D=\frac{1}{2} T_{i}^{i}$, Lorentz group $=\operatorname{traceless} T_{j}^{i}$, Momentum $=S^{i j}$, special conformal $=S_{i j}$.

$S O(5)$ may be constructed in an analogous fashion. Its maximal subgroup is $S O(3) \times U(1)=U(2)$. The remaining generators of $S O(5)$ consist of $2 S O(3)$ vectors, linear combinations of which have weight \pm 1 under the $U(1)$ and so are symmetric tensors in the $2 / 2^{*}$ representation of $U(2)$. The construction of the $S O(5)$ algebra is almost identical to the construction of $S O(6,2)$, with $i, j$ indices being replaced by $\mu, \nu$ indices that run from 1-2, bosons replaced by fermions and antisymmetric tensors replaced by symmetric tensors

$$
\begin{aligned}
& M_{\nu}^{\mu}=\alpha^{\mu} a_{\nu}-b_{\nu} b^{\mu} \\
& A_{\mu \nu}=\alpha_{\mu} \beta_{\nu}+\alpha_{\nu} \beta_{\mu} \\
& A^{\mu \nu}=\alpha^{\mu} \beta^{\nu}+\alpha^{\nu} \beta^{\mu}
\end{aligned}
$$

\footnotetext{
${ }^{57}$ Construction with an odd number of oscillators turns out not to be possible
} 
Oscillator representations of $S O(5)$ are easily constructed. Representations are decomposed into states of equal weight under $Y=\frac{1}{2} M_{\mu}^{\mu}$, which appear in multiplets of $S O(3)$. The $Y$ value and the spin $s$ of the $S O(3)$ representation of the lowest weight state label representations. It is easy to translate to the more conventional GZ highest weight labeling of $S O(5)$ representation : $\left(l_{1}, l_{2}\right)=(-x, s)$.

The full Lie super algebra $\mathrm{SO}(6,2 / 2)$ may be constructed easily using oscillators introduced in the two paragraphs above. Define 2 super tuples of oscillators:

$$
A_{m}=\left(a_{i}, \alpha_{\mu}\right) ; B_{m}=\left(b_{i}, \beta_{\mu}\right)
$$

The generators of $\mathrm{SO}(6,2 / 2)$ may be constructed with $n=2 p$ families of oscillators as

$$
\begin{gathered}
M_{n}^{m}=A^{m} A_{n}+(-1)^{\operatorname{deg}(m) \operatorname{deg}(n)} B_{n} B^{m} \\
S_{m n}=A_{m} B_{n}+A_{n} B_{m} \\
S^{m n}=A^{m} B^{n}+A^{n} B^{m}
\end{gathered}
$$

The even part of the superalgebra is $S O(6,2) \times S O(5)$; our construction on the even part reduces to the constructions of these algebras presented above. Odd elements of the superalgebra carry a single bosonic $(U(4))$ index, and one fermionic $(U(2))$ index. Odd elements with raised / lowered bosonic indices are identified with $Q^{\prime} s / S^{\prime} s$, the generators of supersymmetry, and super special conformal transformations, in the algebra. ${ }^{58}$

Representations of the full supergroup $S O(4,2 / 2)$ obtained by the oscillator method are naturally decomposed into states of equal weight under $\frac{1}{2} M_{m}^{m}=D+Y=K$, and appear in multiplets of the supergroup $U(4 / 2)$. Representations are labelled by the $U(2 / 4)$ multiplet that states with the lowest $K$ value transform in. Translation into the more usual labeling (of the lowest $D$ value appearing in the representation, and the $S O(6) \times S O(5)$ representation that states with this $D$ value transform in), is easy. $U(4 / 2)$ representations representations consist of a finite number of representations of $U(4) \times U(2)$. The whole representation is uniquely specified by the $U(4) \times U(2)$ representation in this group that has lowest $\epsilon_{0}$. With this understanding $U(4 / 2)$ representations are labeled by

\footnotetext{
${ }^{58}$ Once again this identification is in the sense of sec 2.2
} 
$U(4) \times U(2)=U_{D}(1) \times S O(6) \times U_{Y}(1) \times S O(3)$ weights. We want to transform this into a set of $U_{D}(1) \times S O(6) \times S O(5)$ labels - that is easily achieved. $U(1)_{D}$ and $S O(6)$ labels map into each other: $U_{Y}(1) \times S O(3)$ labels map into $S O(5)$ weights according to the formulae presented above when analysing representations of $S O(5)$.

Consider some specific representations of this algebra that may be constructed using this method.

C1) Representations constructed using $n=2 p$ oscillators, and with the fock space vacuum chosen as lowest weight state, possess a lowest weight state that transforms as a singlet under $U(4 / 2)$, and with weights $\epsilon_{0}=D=$ $2 p ; Y=-p$. The lowest $D$ weight of these states is, therefore, $\epsilon_{0}=2 p$; states with this scaling dimension transform as scalars under $S O(6)$ but in the $(p, 0) \mathrm{GZ}$ highest weight representation of $S O(5)$. These are the short representations of this algebra that appear $\mathrm{n}$ the field theory of the $M_{5}$ brane as explained behind.

C2) Representations constructed using $n=2 p$ oscillators, and with lowest weight states $=\alpha_{(1)}^{\mu} \mid 0>>^{59}$ have $\epsilon_{0}=2 p$, are $S O(6)$ scalars and transform in $S O(5)$ under $\left(p-\frac{1}{2}, \frac{1}{2}\right)$. These are also examples of short representations with $\epsilon_{0}=2\left(l_{1}+l_{2}\right)$.

C3) Representations constructed using $n=2 p$ oscillators, and with lowest weight states $=\alpha_{(1)}^{\nu} \alpha_{(1)}^{\mu} \mid 0>$, have $\epsilon_{0}=2 p$, are $S O(6)$ scalars and transform in $S O(5)$ under $(p-1,0)$. These are examples of short representations with $h_{1}=h_{2}=h_{3}=0$ and $\epsilon_{0}=2\left(l_{1}+l_{2}\right)+2$.

C4) Representations constructed using $n=2 p$ oscillators, and with low-

\footnotetext{
${ }^{59}(1)$ is a 'which oscillator' index. Here and in the constructions given below, one really has in mind the following. One constructs a $U(4 / 2)$ multiplet lowest weight state by acting on fock space by a set of super oscillator raising operators with some symmetry properties between them. One then studies the states produced and picks out those with the lowest $\epsilon_{0}$ value. One could not have, for instance, considered the state $a^{i} \mid 0>$ as a lowest weight state because that state appears in the same multiplet as the state $\alpha^{\mu} \mid 0>$ and the later has lower $\epsilon_{0}$ weight. As a less trivial example of a lowest weight state, consider the state formed when $n+2$ super oscillators, each of flavour 1 , are super symmetrized, and act on the fock space. The lowest weight state has 2 of the oscillators being $\alpha$ mutually antisymmetrized ( only 2 because you cannot anti symmetrize more than $2 \alpha s$ of a given flavour), and the rest of the oscillators as as symmetrized. This is the situation in C5) below. The multiplets to which the lowest states in the remaining constructions below belong to are : C3) 2 super oscillators of the same flavour, supersymmetrized acting on fock vacuum. C4) 2 super oscillators of flavour 1 supersymmetrized and 2 super oscillators of flavour 2 supersymmetrized acting on vacuum. C6) $n+2$ oscillators of flavour 1 supersymmetrized and 2 oscillators of flavour 2 supersymmetrized acting on fock vacuum
} 
est weight states $=\alpha_{(1)}^{\nu} \alpha_{(1)}^{\mu} \alpha_{(2)}^{\phi} \alpha_{(2)}^{\theta} \mid 0>{ }^{60}$, have $\epsilon_{0}=2 p$, are $S O(6)$ scalars and transform in $S O(5)$ under $(p-2,0)$. These are examples of short representations with $h_{1}=h_{2}=h_{3}=0$ and $\epsilon_{0}=2\left(l_{1}+l_{2}\right)+4$.

C5) Representations constructed using $n=2 p$ oscillators, and with lowest weight states $=\alpha_{(1)}^{\nu} \alpha_{(1)}^{\mu} a_{(1)}^{i_{1}} a_{(1)}^{i_{2}} \ldots a_{(1)}^{i_{n}} \mid 0>$, have $\epsilon_{0}=2 p+\frac{n}{2}, S O(6)$ weights $\left(\frac{n}{2}, \frac{n}{2}, \ldots \frac{n}{2}\right)$, and transform in $S O(5)$ under $(p-1,0)$. These are examples of short representations with $h_{1}=h_{2}=h_{3}=\frac{n}{2}$ and $\epsilon_{0}=h_{1}+2\left(l_{1}+l_{2}\right)+2$.

C6) Representations constructed using $n=2 p$ oscillators, and with lowest weight states $=\alpha_{(1)}^{\nu} \alpha_{(1)}^{\mu} \alpha_{(2)}^{\phi} \alpha_{(2)}^{\theta} a_{(1)}^{i_{1}} a_{(1)}^{i_{2}} \ldots a_{(1)}^{i_{n}} \mid 0>{ }^{61}$, have $\epsilon_{0}=2 p+\frac{n}{2}$ , have $S O(6)$ weights $\left(\frac{n}{2}, \frac{n}{2}, \ldots \frac{n}{2}\right)$ and transform in $S O(5)$ under $(p-2,0)$. These are examples of short representations with $h_{1}=h_{2}=h_{3}=\frac{n}{2}$ and $\epsilon_{0}=h_{1}+2\left(l_{1}+l_{2}\right)+4$.

In summary, using the oscillator method we have managed to construct all the short representations of the $d=6, n=1$ algebra whose existence we were in doubt about. Since the $d=6, n=2$ algebra contains a $d=$ $6, n=1$ sub-algebra, this construction has established the existence of the corresponding representations for $d=6, n=1$ as well.

\section{Results}

\section{Representations of the Conformal Algebra}

Representations of the conformal algebra in spacetime dimension $d$ are labeled by a scaling dimension $\epsilon_{0}$ and $S O(d)$ weights $\left(h_{1}, h_{2}, . . h_{[d / 2]}\right)$. Necessary conditions for these representations to be unitary are

$$
\epsilon_{0} \geq \frac{d-2}{2}
$$

for the scalar representation (all $h \mathrm{~s}=0$ );

$$
\epsilon_{0} \geq \frac{d-1}{2}
$$

for the spinor representation.

$$
\epsilon_{0} \geq d-1
$$

for the vector representation. And

$$
\epsilon_{0} \geq\left|h_{i}\right|+d-i-1
$$

\footnotetext{
${ }^{60}$ Of course this construction makes sense only for $p \geq 2$

${ }^{61}$ Of course this construction makes sense only for $p \geq 2$
} 
for any other representation $\mathrm{R}$, where $i$ is the smallest value s.t. $h_{i} \geq\left|h_{i+1}\right|+$ 1 , if there exists such an $i$, or is equal to $[d / 2]$ ([ ] takes integer part), if there is no such $i$.

These conditions are also sufficient to guarantee unitarity in $d=3,4$, according to [1], [2] . I have not attempted to find sufficient conditions in arbitrary dimension.

Free representations of the conformal algebra, in the form of a local operator obeying a free wave equation, exist only when ${ }^{62}$

a) $\mathrm{d}=$ even. $h_{1}=h_{2}=\ldots=\left|h_{[d / 2]}\right|=h$. In that case $\epsilon_{0}=\frac{d-2}{2}+h$.

b) d=odd. $h_{1}=h_{2}=\ldots=h_{[d / 2]}=h$ and $h=0$ or $h=\frac{1}{2}$. In that case $\epsilon_{0}=\frac{d-2}{2}+h$

\section{Unitary Representations of Superconformal Algebras $\mathrm{d}=3$}

Representations are labeled by and $S O(3)$ weight $\mathrm{j}$, and by $h_{1} \ldots h_{n / 2}$, $S O(n)$ highest weights and a lowest dimension $\epsilon_{0}$. We report results for even $\mathrm{n}$.

If $\mathrm{j}>0$ then the condition $\epsilon_{0} \geq j+1+h_{1}$ is necessary and sufficient to guarantee unitarity. A representation that saturates this equality in $\epsilon_{0}$ is in a short representation of the Superconformal Algebra, and so the scaling dimension of the operator is likely to remain constant as one turns interactions on or off in the theory.

If $\mathrm{j}=0$ then unitary representations exist for $\epsilon_{0}=h_{1}$ and for $\epsilon_{0} \geq h_{1}+1$. The representation at the isolated value of $\epsilon_{0}$, and the one at the value of $\epsilon_{0}$ that saturates the inequality above, are short.

$$
\begin{aligned}
& \text { If } \mathrm{n}=1 \text { then } \epsilon_{0} \geq \frac{1}{2} \text { if } j=0 \text { and } \epsilon_{0} \geq j+1 \text { otherwise. } \\
& \qquad \mathbf{d}=\mathbf{4}
\end{aligned}
$$

These results were first obtained by Dobrev and Petkova in [3] . Representations are labeled by $2 S U(2)-$ Lorentz - js, by a $U(1) \mathrm{R}$ charge $\mathrm{e}^{63}$ and by an $S U(n)$ representation - which we label by positive integers $r_{k}$, the number of boxes in the $k^{\text {th }}$ row of the Young Tableaux.

If $j_{1} \neq 0 \neq j_{2}$ then $\epsilon_{0} \geq \max \left(d_{n n}^{\prime 3}, d_{n 1}^{\prime 1}\right)$ where $d^{\prime}$ s are defined in equationss

\footnotetext{
${ }^{62}$ this result was obtained largely in [13] .

${ }^{63}$ For reasons mentioned in Section 4 the case $n=4$ is special. In this case the simple LSA has no $\mathrm{R}$ charge in it - the $\mathrm{R}$ symmetry group is $S U(n)$. The results stated here all hold on setting $R=0$ in all formulae
} 
4.34 - 4.37 . These conditions are necessary and sufficient to guarantee unitarity. A representation with dimension saturating the inequality is a short representation.

If $j_{1}=0, j_{2} \neq 0$ and $d_{n n}^{\prime 3}>d_{n 1}^{\prime 2}$ then the condition above continues to be necessary and sufficient to guarantee unitarity.

If $j_{2}=0, j_{1} \neq 0$ and $d_{n n}^{\prime 4}<d_{n 1}^{1}$ then the condition above continues to be necessary and sufficient to guarantee unitarity.

If $j_{1}=0, j_{2} \geq 0$ and $d_{n n}^{\prime 3} \leq d_{n 1}^{\prime 2}$ then $\epsilon_{0} \geq d_{n 1}^{\prime 1}$ is sufficient to guarantee unitarity. It is not however $n$ ecessary - there exists a (short representation) at $\epsilon_{0}=d_{n 1}^{\prime 2}$ that is unitary. There are no other exceptions.

If $j_{2}=0, j_{1} \geq 0$ and $d_{n n}^{\prime 4} \geq d_{n 1}^{\prime 1}$ then $\epsilon_{0} \geq d_{n n}^{\prime 3}$ is sufficient to guarantee unitarity. It is not however necessary - there exists a (short representation) at $\epsilon_{0}=d_{n n}^{\prime 4}$ that is unitary.

If $j_{1}=j_{2}=0$, then unitary representations exist for $\epsilon_{0} \geq \max \left(d_{n n}^{\prime 3}, d_{n 1}^{\prime 1}\right)$ and for $\epsilon_{0}=d_{n 1}^{\prime 2}$ if $d_{n 1}^{\prime 2} \geq d_{n n}^{\prime 3}$ and for $\epsilon_{0}=d_{n n}^{\prime 4}$ if $d_{n n}^{\prime 4} \geq d_{n 1}^{\prime 1}$ and for $\epsilon_{0}=d_{n 1}^{\prime 2}$ if $d_{n 1}^{\prime 2}=d_{n n}^{\prime 4}$. Unitary representations exist for no other values of $\epsilon_{0}$, except for the vacuum representation dealt with below.

Finally if all labels $j_{1}, j_{2}, r_{i}, R$ are zero then there exists in addition to the representations above, a trivial one dimensional representation.

$$
\mathrm{d}=\mathbf{5}
$$

Representations are labeled by scaling dimension $\epsilon_{0}$, by an $\mathrm{R}$ symmetry $(S U(2))$ half integer $k$, and by $S 0(5)$ (Lorentz) weights $\left[h_{1}, h_{2}\right]$.

If $h_{1} \neq 0 \neq h_{2}$ then $\epsilon_{0} \geq h_{1}+h_{2}+4+3 k$ forms the necessary and sufficient conditions for the occurrance of unitary representations. The representation at the saturating dimension is short.

If $h_{1} \neq 0, h_{2}=0$ then there do exist unitary representations for $\epsilon_{0} \geq$ $h_{1}+3 k+4$. The only other dimension at which a unitary representation may exist is $\epsilon_{0}=h_{1}+3 k+3$. My calculations suggest, but do not prove, that a unitary representation exists at this isolated value of $\epsilon_{0}$. The representation at the dimension that saturates the inequality, and at the isolated dimension (if it exist) are short representations.

If $h_{1}=h_{2}=0$ then unitary representations exist for $\epsilon_{0} \geq 3 k+4$. The only other values of $\epsilon_{0}$ at which unitary representations could possibly exist are $\epsilon_{0}=3 k$ and $\epsilon_{0}=3 k+3$. My calculations suggest, but do not prove, that unitary representations do indeed exist at these isolated values of $\epsilon_{0}$. The 
representation at the value of $\epsilon_{0}$ that saturates the inequality, and at those at the isolated values of $\epsilon_{0}$, (if they exist), are short representations.

$$
\mathrm{d}=6
$$

Supersymmetry generators are taken to be chiral spinors, i.e., $\left(h_{1}, h_{2}, h_{3}\right)=$ $\left(\frac{1}{2}, \frac{1}{2}, \frac{1}{2}\right)$.

$$
\mathrm{n}=1
$$

Representations are labeled by scaling dimension $\epsilon_{0}$, by an $S U(2) \mathrm{R}$ symmetry half integer $k$, and by $S 0(6)$ (Lorentz) weights $\left[h_{1}, h_{2}, h_{3}\right]$.

a) $h_{2}-\frac{1}{2} \geq\left|h_{3}+\frac{1}{2}\right|$ Unitary representations exist if and only if $\epsilon_{0} \geq$ $h_{1}+h_{2}-h_{3}+4 k+6$ The representations at the value of $\epsilon_{0}$ that saturates this inequality is short.

b) $h_{1}-h_{2} \geq 1$ and the condition of part a) is not met. Unitary representations do exist when $\epsilon_{0} \geq h_{1}+h_{2}-h_{3}+4 k+6$. The representation at the dimension that saturates this bound is short. The only other value of $\epsilon_{0}$ at which unitary representations may exist are $\epsilon_{0}=h_{1}-h_{2}+h_{3}+4 k+4$. My calculations suggest, but do not prove the existence of a unitary representation at this dimension. If this representation exists, it is short.

c) $h_{1}=h_{2}=h_{3}=h \neq 0$ Unitary representations do exist for $\epsilon_{0} \geq$ $6+h+4 k$. The representation at $\epsilon_{0}=6+h+4 k$ is short. Short unitary representations also exist for $\epsilon_{0}=2+h+4 k, 4+h+4 k$. Unitary representations may or may not exist for $\epsilon_{0}$ in the interval $(2+h+4 k, 4+h+4 k)^{64}$.

d) $h_{1}=h_{2}=h_{3}=0$ Unitary representations do exist for $\epsilon_{0} \geq 4 k+6$. The representation at $\epsilon_{0}=4 k+6$ is short. Short unitary representations also exist for $\epsilon_{0}=4 k, 4 k+2,4 k+4$. Unitary representations may or may not exist for $\epsilon_{0}$ in the interval $(4 k+2,4 k+4){ }^{65}$.

$$
\mathrm{n}=2
$$

Representations are labeled by scaling dimension $\epsilon_{0}$, by an R symmetry $(S O(5))$ half weights $l_{1}, l_{2}$, and by $S 0(6)$ (Lorentz) weights $\left[h_{1}, h_{2}, h_{3}\right]$.

Our results are exactly those for $\mathrm{n}=1$, with $\mathrm{k}$ being replaced by $\frac{\left(l_{1}+l_{2}\right)}{2}$.

\section{Acknowledgements}

I would like to acknowledge useful discussions with almost every high energy theory student at the physics department at Princeton, especially R.Gopakumar, M.Krogh, S.Lee and A.Mikhailov (who suggested the use of characters in appendix 2). I would like to thank R.Gopakumar and

\footnotetext{
${ }^{64} \mathrm{I}$ am tempted to guess that representations exist through the interval

${ }^{65} \mathrm{I}$ am tempted to guess that representations exist throughout the interval
} 
M.Rangamani for reading a preliminary draft of this paper and suggesting improvements. I would like to thank V.Dobrev for responding promptly and lucidly with clarifications on his work, and for drawing my attention to [5] . I am grateful to M. Gunaydin for a communication that drew my attention to his work on the oscillator representations of superalgebras. A conversation with K. Intrilligator and another with C.Callan helped clarify some aspects related to section 2. Finally, I would like especially to acknowledge the large contribution of N.Seiberg to this paper, in suggesting the problem, and in providing guidance and advice during every stage of its completion.

\section{Appendix 1: $U(n)$ and $S U(n)$}

We specify here the conventions we use when dealing with the R symmetry $U(n)$ in the case $d=4$.

Consider the matrices $\left(T_{i j}\right)_{p q}=\delta_{i p} \delta_{j q}$. Complex linear combinations of these matrices $T_{i j}+T_{j i}$ and $(-i)\left(T_{i j}-T_{j i}\right)$ are hermitian, and so form a basis for the defining representation of the Lie Algebra of $U(n)$. We will find it convenient to work with the $\mathrm{T}$ matrices themselves and their images in every $U(n)$ representation, rather then their Hermitian linear combinations $T_{i j}$. They obey commutation relations.

$$
\left[T_{i j}, T_{m n}\right]=T_{i n} \delta_{j m}-T_{j m} \delta_{i n}
$$

Note $T_{i j}^{\dagger}=T_{j i}$ If $Q_{i}$ is an operator that transforms in the defining representation of $U(n)$, then it obeys

$$
\left[T_{i j}, Q_{m}\right]=\delta_{j m} Q_{i}
$$

If $S_{i}$ transforms in the antifundamental representation of $U(n)$, then

$$
\left[T_{i j}, S_{m}\right]=-\delta_{i m} S_{j}
$$

Therefore the $i$ in $T_{i j}$ is a fundamental index, the $j$ an antifundamental index.

The various weights of the fundamental representation are defined as $\nu_{i}^{\prime}(i=1, \ldots, n)$. If a state $\psi$ has weight $\Sigma a_{i} \nu_{i}^{\prime}$ then $T_{m m} \psi=a_{m} \psi$ (no sum over m). $T_{m m}$, thus, are natural Cartan Generators for $U(n)$. 
Define $R=\Sigma T_{m m}$. $\mathrm{R}$ is a generator for the $U(1)$ sub-algebra of $U(n)$. In the defining representation it is the identity matrix. All vectors in the defining representation have $\mathrm{R}$ charge 1 . All vectors in the antifundamental representation have $\mathrm{R}$ charge -1 . All Ts commute with $\mathrm{R}$, i.e., have zero $\mathrm{R}$ charge.

Consider the decomposition of $U(n)$ into $U(1) \times S U(n)$. The $U(1)$ generator is R above. $S U(n)$ generators may be chosen to be all off diagonal $T s$, and $T_{m}^{\prime}=T_{m m}-R / n$ (no sum over $\mathrm{m}$ ). The $\mathrm{n} T^{\prime} s$ clearly obey the constraint $\Sigma T_{i}^{\prime}=0$. Therefore we regard $T_{i}^{\prime}$ for $i=1 . . n-1$ as the Car$\tan$ generators of our $S U(n)$ algebra. Let $\nu_{i}=\nu_{i}^{\prime}$ restricted in their action to the Cartan generators of $S U(n)$ rather than $U(n)$. Then the arbitrary $S U(n)$ weight vector may be written as $\sum_{i=1}^{n-1} r_{i} \nu_{i}=\sum_{i=1}^{n-1} R_{i} \mu_{i}$ where we have defined $\mu_{i}=\sum_{j=1}^{i} \nu_{j}$. Here $r_{i}=R_{i}-R_{i-1}$. $\mu_{j}$ s are fundamental weights for $S U(n)$. Sums are taken only to $n-1$ because of the identity $\sum_{i=1}^{n} \nu_{i}=0$. When the highest weight of a representation is written in this form, $R_{i}$ refers to the number of columns of length $i$ in the Young Tableaux for that representation, $r_{i}$ refers to the number of boxes in the ith row of the Tableaux.

Acting on a state with weights specified by $r_{i}$, our $T^{\prime}$ operators yield $T_{m}^{\prime} \psi=\left(r_{m}-\frac{\Sigma r_{i}}{n}\right) \psi$ We define the ordering convention $\nu_{1} \geq \nu_{2} \geq \nu_{3} \ldots \geq \nu_{n-1}$ $T_{i j}$ are raising and lowering operators, with roots $\nu_{i}-\nu_{j}$. Therefore $T_{i j}$ is a raising operator for $i \leq j$ and a lowering operator otherwise.

I define a $U(n)$ Casimir of a representation by $C(R)=\sum_{i, j=1}^{n}\left(T_{i j} T_{j i}\right)$. This may now be computed as a function of the highest wts of the $S U(n)$ part of the representation, and of the $\mathrm{R}$ charge of the representation (by acting the casimir on the highest weight state, and noting that all nonzero elements in the sum may be replaced by commutators). The answer is $C(R)=\sum_{i=1}^{n}\left(r_{i}-\right.$ $\left.\Sigma\left(r_{j}\right) / n\right)\left(r_{i}-\Sigma\left(r_{j}\right) / n-2 i\right)+\frac{R^{2}}{n}$ For the purposes of the formula above, we define $r_{n}=0$. As a check on the formula, we note that in the case of $S U(2)$ $T_{i j} T_{j i}=2\left(J^{2}\right)+R^{2} / 2$, and so independently we expect the casimir to be given by $2(j)(j+1)+R^{2} / 2$. Setting $r_{1}=2 j, r_{2}=0$ in the formula above, that is exactly what we get. Again, the Casimir for the fundamental representation should be $\mathrm{n}$ (from definition), again this is reproduced by our formula.

The similarly defined Casimir for $S U(n)$ is obtained by setting $\mathrm{R}=0$ in the formula above. 


\section{Appendix 2: Decomposition of Vector $\times R$ and Spinor $\times R$ for $S O(N)$.}

In this appendix we will derive the irreducible representation content of Vector $\times($ Rep) and Spinor $\times$ rep in $S O(N)$.

Define the character of a representation to be

$$
\chi\left(\left\{h_{j}\right\}, t_{j}\right)=\operatorname{Tr}\left(\exp \left(t_{i} H_{i}\right)\right)
$$

in that representation where $\left\{h_{i}\right\}$ are the highest weights labeling the representation, $t_{i}$ are real variables, and $H_{i}$ are the Cartan Generators of our group in the standard basis. The trace above is taken in the representation labeled by $\left\{h_{i}\right\}$. We sum over $i$ in the RHS of the formula above. Since the character of a product representation must be equal to the product of its factor characters, and must also be equal to the sum of the characters of the its irreducible representation content, the character is a useful aid to performing a Clebsch Gordan decomposition.

$$
\operatorname{SO}(2 \mathrm{n}+1)
$$

The character of the vector representation is clearly given by $1+\sum_{i=1}^{n} 2 \operatorname{Cosh}\left(t_{i}\right)$. The character of the spinor is given by $1+\Pi_{i} 2 \operatorname{Cosh}\left(t_{i}\right)$. The product of the general representation is given by the famous Weyl formula

$$
\chi\left(h_{i}, t_{i}\right)=\frac{\operatorname{det}\left(\sinh \left[t_{i}\left(h_{j}+(n-j)+\frac{1}{2}\right)\right]\right)}{\operatorname{det}\left(\sinh \left[t_{i}\left((n-j)+\frac{1}{2}\right)\right]\right)}
$$

The product of the character of an arbitrary representation with that of the vector representation, leaves the denominator in the 8.2 unchanged, but turns the numerator into the sum of $2 n+1$ determinants, one being the original determinant and the others being obtained from the original determinant by taking $h_{i}$ to $h_{i}+1$ for any given $\mathrm{i}=\mathrm{j}$, leaving $h_{i}$ unchanged for $\mathrm{i}$ $\neq \mathrm{j}$. Note that for any rep $h_{1} \geq h_{2} \geq h_{3} \ldots \geq h_{n} \geq 0$. Of the $2 \mathrm{n}$ determinants we obtain by the process described above not all will be nonzero - only those whose new $h_{i}$ values are ordered as above will yield a nonzero determinant. For instance if originally $h_{1}=h_{2}$, and you form a new determinant with $h_{2}$ augmented by unity, then two rows of the matrix whose determinant is being considered become identical, and the determinant is zero. Similarly, if you form a new determinant with $h_{1}$ decreased by unity you get zero.

Thus we obtain a simple rule : $\left\{h_{i}\right\} \times($ vector $)=\left\{h_{i}\right\}+$ All reps obtained from $\left\{h_{i}\right\}$ by adding or subtracting unity from a single $\mathrm{h}$, provided that the set $\left\{h_{i}\right\}$ thus obtained continues to obey $h_{1} \geq h_{2} \geq h_{3} \geq \ldots \geq h_{n} \geq 0$. 
The rule for the spinor is derived as easily, and I state it below. : $\left\{h_{i}\right\} \times$ $($ spinor $)=$ All reps obtained from $\left\{h_{i}\right\}$ by either adding or subtracting $1 / 2$ from every $h_{i}$, provided that the set $\left\{h_{i}\right\}$ thus obtained continues to obey $h_{1} \geq h_{2} \geq h_{3} \ldots \geq h_{n} \geq 0$.

This rule could lead to a maximum of $2^{n}$ representations.

\section{$\mathrm{SO}(2 \mathrm{n})$}

A condition $S O(2 n)$ highest weights must obey is $h_{1} \geq h_{2} \geq h_{3} \ldots\left|h_{n}\right| \geq 0$. (recall $h_{n}$ may be negative). The Weyl formula for $S O(2 n)$ is

$$
\chi\left(h_{i}, t_{i}\right)=\frac{\operatorname{det}\left(\sinh \left[t_{i}\left(h_{j}+n-j\right)\right]\right)+\operatorname{det}\left(\cosh \left[t_{i}\left(h_{j}+n-j\right)\right]\right)}{\operatorname{det}\left(\sinh \left[t_{i}(n-j)\right]\right)}
$$

Where the character is defined as for $S O(2 n+1)$. The vector and 2 spinor characters may be worked out from the formula above. Redoing our $S O(2 n+1)$ computation leads to the following rules.

$:\left\{h_{i}\right\} \times($ vector $)=$ All reps obtained from $\left\{h_{i}\right\}$ by adding or subtracting unity from a single $\mathrm{h}$, provided that the set $\left\{h_{i}\right\}$ thus obtained continues to obey $h_{1} \geq h_{2} \geq h_{3} \geq \ldots \geq\left|h_{n}\right| \geq 0$.

$:\left\{h_{i}\right\} \times \pm$ chirality spinor $=$ All reps obtained from $\left\{h_{i}\right\}$ by either adding or subtracting $\frac{1}{2}$ from every $h_{i}$, the number of subtractions being (even/odd), provided that the set $\left\{h_{i}\right\}$ thus obtained continues to obey $h_{1} \geq$ $h_{2} \geq h_{3} \geq \ldots \geq\left|h_{n}\right| \geq 0$.

\section{References}

[1] N.T.Evans, "Discrete Series for the Universal Covering Group of the 3+2 dimensional de Sitter Group",J.Math.Phys. 8 (1967) 170-185.

[2] G. Mack, "All Unitary Ray Representations of the Conformal Group $\mathrm{SU}(2,2)$ with Positive Energy,Comm. Math. Phys. 55 (1977) 1"

[3] V.K.Dobrev and V.B. Petkova "All Positive Energy Unitary Irreducible Representations Of Extended Conformal Supersymmetry,"Phys. Lett. B162B (1985) 127-132

[4] V.K.Dobrev and V.B. Petkova, "Group Theoretic Approach To Extended Conformal Supersymmetry...," Fortschr. Phys. 35 (1987) 537572 , The relevant portions are contained mainly in section 7 
[5] V.K.Dobrev and V.B. Petkova, "All Positive Energy Unitary Irreducible Representations Of Extended Conformal Supersymmetry," in 'Conformal Groups and Related Symmetries Physical Results and Mathematical Background, Springer Verlag Lecture notes in Physics 261, Proceedings of a Symposium at Clausthal, Eds A.O.Barut and H.D. Doebner

[6] V.G.Kac, "Representations of Classical Lie Superalgebras", in Lecture Notes in Mathematics, Vol. 676 (Springer-Verlag, Berlin, 1978) 597

[7] V.G.Kac, "Lie Superalgebras", Advances in Mathematics, 26 (1977), 8-96.

[8] Steven Shnider, "The Superconformal Algebra in Higher Dimensions "Let. Math. Phys. 16 (1988) 377-383.

[9] M. Scheunert, W.Nahm and V. Rittenberg, "Classification of allsimple graded Lie algebras whose Lie algebra is reductive. 2. Construction of the exceptional algebras" J.Math.Phys. 17 (1976) 1640-1644, see appendix C.

[10] W. Heidenreich, "All Linear Unitary Irreducible Representations of de Sitter Supersymmetry with Positive Energy," Phys. Lett. B110 (1982) 461-464.

[11] D.Z.Friedman and H.Nicolai, "Multiplet Shortening in $\operatorname{Osp}(\mathrm{N}, 4)$ ", Nucl. Phys. B237 (1984) 342-366

[12] J.Strathdee, "Extended Poincare Supersymmetry", Int. J. of Modern Physics A, 2 (1987) 273-300

[13] W.Siegel, "All Free Conformal representations in all Dimensions," Int. J. of Modern Physics A, 4 (1989) 2015-2020

[14] Two references on the rather strange fact that scale invariance often implies conformal invariance are - S.Coleman, "Aspects of Symmetry", Cambridge University Press, Lecture 3; and J. Polchinksi, "Scale and Conformal invariance in Quantum Field Theory", Nucl. Phys. B303 (1988) 226-236

[15] Nathan Seiberg, "Notes on Theories with 16 Supercharges", hepth/9705117

[16] A.Casher, F.Englert, H. Nicolai , M.Rooman, "The Mass Spectrum of Supergravity on the round seven sphere", Nucl. Phys. B243 (1984) 173-188 
[17] M.Gunaydin and N.P. Warner, "Unitary Supermultiplets of $\operatorname{OSp}(8 / 4, R)$... Supergravity," Nucl. Phys. B272 (1986) 99-124

[18] M.Gunaydin and N. Marcus, "The Spectrum of the $S^{5}$ compactification of the Chira $\mathrm{N}=2, \mathrm{D}=10 \ldots \mathrm{U}(2,2 / 4)$," Class. Quantum Grav. 2 (1985) L11-L17

[19] M.Gunaydin and P. van Nieuwenhuizen, and N.P Warner, "General construction of the unitary representaion of $A d S$ superalgebras and the spectrum of the $S^{7}$ compactification of 11-dimensional Supergravity " Nucl. Phys. B255 (1985) 63-92

[20] H.J.Kim, L.J. Romans and P.Nieuwenhuizen, "Mass Spectrum of chiral 10d N=2 supergravity on $S^{5}$, " Phys. Rev. D32 (1985) 389-399

[21] H.J.Kim, L.J. Romans and P.Nieuwenhuizen, "Mass Spectrum of chiral 10d N=2 supergravity on $S^{5}$, " Phys. Rev. D32 (1985) 389-399

[22] P.Claus, R. Kallosh and A V Proeyen, "M-5 brane and superconformal $(0,2)$ tensor multiplet in 6 dimensions ," hep-th/9711161 